\title{
Sierra Leone: Fifth Review Under the Arrangement Under the Poverty Reduction and Growth Facility, Request for Waiver of Nonobservance of Performance Criterion, Request for Modification of Performance Criteria, and Financing Assurances Review- Staff Report; Staff Supplement; Press Release and ED's Statement
}

In the context of the Fifth Review Under the Arrangement Under the Poverty Reduction and Growth Facility, Request for Waiver of Nonobservance of Performance Criterion, Request for Modification of Performance Criteria, and Financing Assurances Review, the following documents have been released and are included in this package:

- $\quad$ The staff report for the Fifth Review Under the Arrangement Under the Poverty Reduction and Growth Facility, Request for Waiver of Nonobservance of Performance Criterion, Request for Modification of Performance Criteria, and Financing Assurances Review, prepared by a staff team of the IMF, following discussions that ended on September 22, 2009, with the officials of Sierra Leone on economic developments and policies. Based on information available at the time of these discussions, the staff report was completed on December 7, 2009. The views expressed in the staff report are those of the staff team and do not necessarily reflect the views of the Executive Board of the IMF.

- $\quad$ A staff supplement.

- A Press Release summarizing the decision taken by the Executive Board to complete the review.

- $\quad$ A statement by the Executive Director for Sierra Leone. *

The documents listed below have been or will be separately released.

Letter of Intent sent to the IMF by the authorities of Sierra Leone*

Memorandum of Economic and Financial Policies by the authorities of Sierra Leone*

Technical Memorandum of Understanding*

*Also included in Staff Report

The policy of publication of staff reports and other documents allows for the deletion of market-sensitive information.

Copies of this report are available to the public from

International Monetary Fund • Publication Services

$70019^{\text {th }}$ Street, N.W. • Washington, D.C. 20431

Telephone: (202) 623-7430 • Telefax: (202) 623-7201

E-mail: publications@imf.org Internet: http://www.imf.org

\section{International Monetary Fund Washington, D.C.}




\section{INTERNATIONAL MONETARY FUND}

\section{SIERRA LEONE}

Fifth Review Under the Arrangement Under the Poverty Reduction and Growth Facility, Request for Waiver of Nonobservance of Performance Criterion, Request for Modification of Performance Criteria, and Financing Assurances Review

Prepared by the African Department

(In consultation with other departments)

Approved by Seán Nolan and Dominique Desruelle

December 7, 2009

Fund relations. The Executive Board approved on May 10, 2006 a three-year PRGF arrangement in an amount equivalent to SDR 31.11 million (30 percent of quota). The arrangement was later extended by one year to May 9, 2010. Two augmentations of access in an amount equivalent to SDR 10.4 million each (about 10 percent of quota) were approved by the Executive Board upon the completion of the third and the fourth review in December 2008 and June 2009, respectively. Five disbursements totaling SDR 32.7 million have been made.

Staff team. The mission comprised Messrs. Mikkelsen, Nsengiyumva, and Million and Ms. Saxena (all AFR). Mr. Atingi-Ego (AFR) joined the mission in the last week.

Mr. Itam (OED) participated in several policy meetings.

Discussions. Discussions were held in Freetown September 9-22, 2009. The mission met with Finance Minister Kamara, Central Bank Governor Sesay, other government officials, and representatives of civil society and donors. 


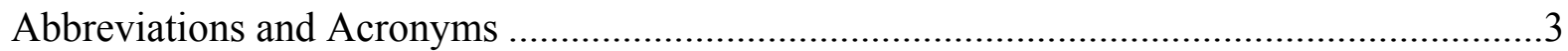

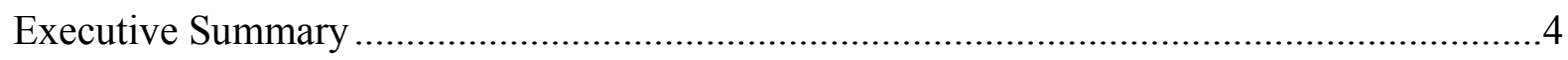

I. Recent Developments and Program Performance .................................................

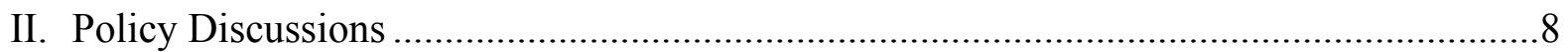

A. Macroeconomic Framework .......................................................................

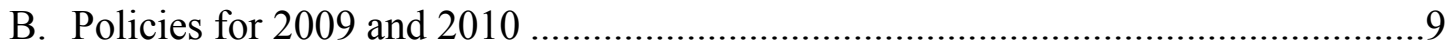

III. Program Risks and Monitoring and Capacity to Repay the Fund .................................13

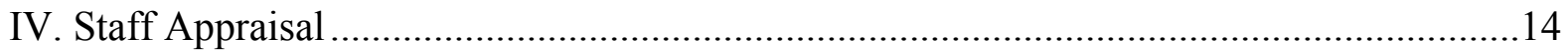

Figure

1. Selected Macroeconomic Indicators, 2005-09........................................................6

Tables

1. Selected Economic and Financial Indicators, 2007-12 ….........................................15

2. Central Government Financial Operations, 2008-12 (Le billions) ................................16

3. Central Government Financial Operations, 2008-12 (Percent of GDP) .........................17

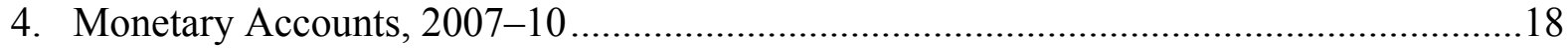

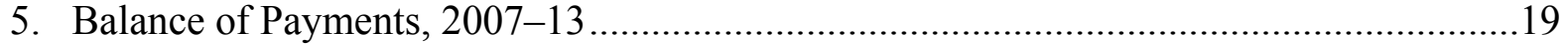

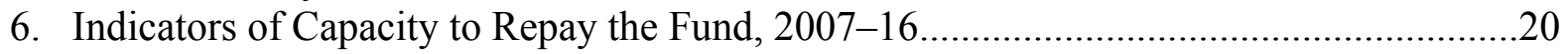

7. Schedule of Disbursements Under the PRGF Arrangement, 2006-10...........................21

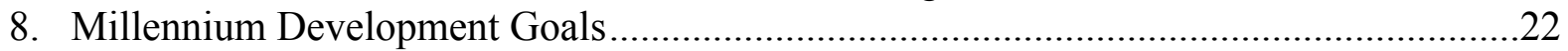

9. Financial Soundness Indicators of the Banking System, 2005-09................................23

Appendix

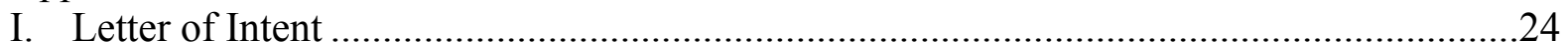

Attachment I. Memorandum of Economic and Financial Policies .................................26

Attachment II. Technical Memorandum of Understanding .........................................35 


\begin{tabular}{|c|c|}
\hline $\mathrm{ACC}$ & Anti-Corruption Commission \\
\hline BOP & Balance of payments \\
\hline BSL & Bank of Sierra Leone \\
\hline CRF & Consolidated revenue fund \\
\hline DSA & Debt Sustainability Analysis \\
\hline $\mathrm{EC}$ & European Commission \\
\hline EU & European Union \\
\hline FAD & Fiscal Affairs Department \\
\hline FSDP & Financial Sector Development Plan \\
\hline GDP & Gross domestic product \\
\hline GST & Goods and services tax \\
\hline HIPC & Highly indebted poor countries \\
\hline IMF & International Monetary Fund \\
\hline LTO & Large Taxpayer Office \\
\hline MDAs & Ministries, departments, and agencies \\
\hline MDG & Millennium Development Goal \\
\hline MDRI & Multilateral Debt Relief Initiative \\
\hline MEFP & Memorandum of Economic and Financial Policies \\
\hline MoFED & Ministry of Finance and Economic Development \\
\hline MPC & Monetary Policy Committee \\
\hline MTEF & Medium Term Expenditure Framework \\
\hline NEER & Nominal effective exchange rate \\
\hline NPA & National Power Authority \\
\hline NPLs & Nonperforming loans \\
\hline NRA & National Revenue Authority \\
\hline $\mathrm{PC}$ & Performance criterion \\
\hline PFM & Public financial management \\
\hline PRGF & Poverty Reduction and Growth Facility \\
\hline PRS & Poverty Reduction Strategy \\
\hline PRSP & Poverty Reduction Strategy Paper \\
\hline PV & Present value \\
\hline REER & Real effective exchange rate \\
\hline SDR & Special drawing rights \\
\hline SPF & Strategic Petroleum Fund \\
\hline TA & Technical assistance \\
\hline TMU & Technical Memorandum of Understanding \\
\hline
\end{tabular}




\section{Executive Summary}

- $\quad$ Program performance in the first half of 2009 was satisfactory. All end-June quantitative performance criteria were met, as were all end-June structural benchmarks, although one with a delay. One of the two structural benchmarks for September 2009 was met, while the other was missed because of delays in revising the underlying legislation.

- The authorities are requesting a waiver for nonobservance of the continuous performance criterion on introduction or modification of multiple currency practices (MCPs) during the period covered by this review. In August 2009, the BSL introduced a special rice window in its weekly foreign exchange auction, which gave rise to an MCP. This rice window was eliminated at end-September 2009, along with an oil window that had been introduced in November 2008. Failure to report to staff the introduction of the oil window resulted in two noncomplying disbursements at the completion of the third (December 2008) and fourth (June 2009) reviews; this issue is covered in a separate memorandum from the Managing Director to the Executive Board.

- The economic slowdown continued in 2009 but there are signs of a turnaround in exports and economic activity. While economic activity was still weak in the first half of 2009, exports of diamonds and agricultural products have recently picked up and real GDP growth should reach 4 percent in 2009. Inflation eased to 8.3 percent as of August 2009, but there are pressures stemming from the sizeable depreciation of the leone this year.

- $\quad$ Fiscal policy for the remainder of 2009 will aim to keep domestic revenue collection on track while containing unanticipated spending pressures. The domestic revenue target is expected to be met, while budget spending is under pressure from unanticipated outlays totaling 0.8 percent of GDP — mainly linked to electricity supply and basic infrastructure projects. Staff agreed with the authorities on a budget framework for 2010 that aims to maintain macroeconomic stability and sustaining economic growth.

- $\quad$ Strengthening tax administration and broadening the tax base remains a challenge. The authorities are moving to make the NRA more efficient and raise taxpayer compliance and are preparing to introduce the GST on January 1, 2010.

- Monetary policy will strive to keep inflation in single digits and contain pressure on the exchange rate. The BSL will continue to conduct open market operations and sales of foreign exchange to mop up excess liquidity. The central bank will limit interventions in the foreign exchange market to smooth out short-term exchange rate volatility.

- Structural reforms will focus on deepening the financial sector; reforming the electricity sector to provide reliable and cost-efficient service throughout the country; enhancing efficiency in the use of public resources; promoting good governance; and establishing a transparent and automatic pricing framework for petroleum products. 


\section{Recent Developments And Program Performance}

1. The global economic downturn continued to weaken economic activity in the first half of 2009, though inflation pressures eased (Figure 1 and Table 1).

- $\quad$ Falling global demand and declining foreign inflows negatively affected economic activity in the first half of 2009. However, diamond and agricultural production has rebounded lately. Although down from 5.5 percent in 2008, real GDP growth is expected to reach 4 percent in 2009.

- Lower fuel prices and higher domestic food production helped bring inflation down from double digits in 2008 to 8.3 percent in August 2009. However, there are upside risks to inflation after the recent depreciation of the leone against the US dollar.

- The external current account deficit jumped to 10.6 percent of GDP in 2008 from 3.4 percent of GDP in 2007 because of the rise in food and fuel prices in the first half of 2008 and plunging diamond exports in the second half. International reserves reached US\$210 million at end2008 (4.5 months of import coverage) and were maintained at this level through June 2009, helped by the PRGF augmentation granted at the conclusion of the fourth review. Sierra Leone's share of the recent SDR allocations,

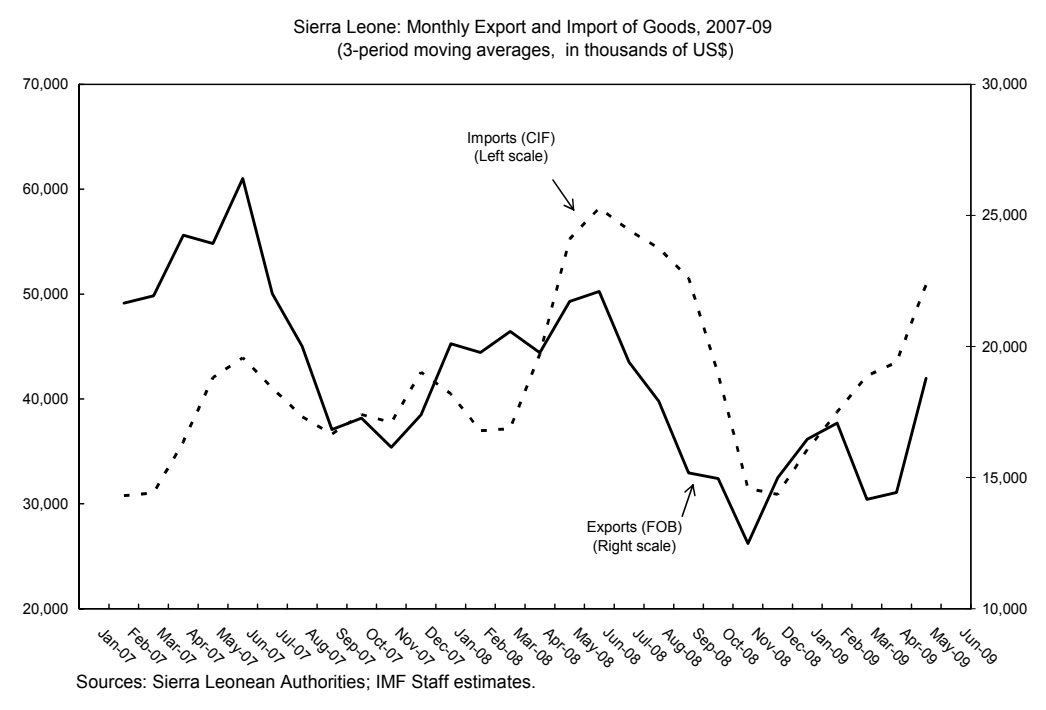
equivalent to about US\$128 million, was allocated to the BSL in late August and early September, bringing gross foreign exchange reserves to about US\$336 million at end-September.

- $\quad$ After remaining stable for the past three years, the leone depreciated by about 20 percent against the US dollar during the first 9 months of 2009, reflecting a decline in foreign exchange inflows. The REER depreciated by about 10 percent during the first seven months of 2009, reversing a third of the appreciation observed in 2008 . $^{1}$

\footnotetext{
${ }^{1}$ The real appreciation in 2008 reflected the large depreciation of the British pound during this period.
} 
Figure 1. Sierra Leone: Selected Macroeconomic Indicators, 2005-09

(Percent of GDP, unless indicated otherwise)

Growth has been robust and broad-based.

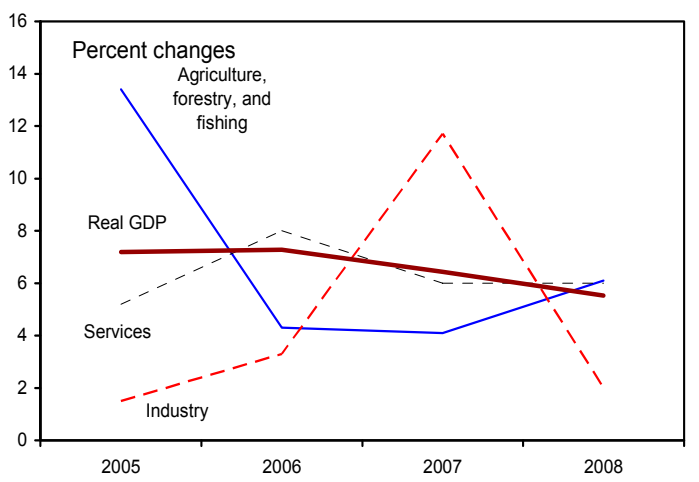

Performance under the program has been hampered by weak revenue mobilization...

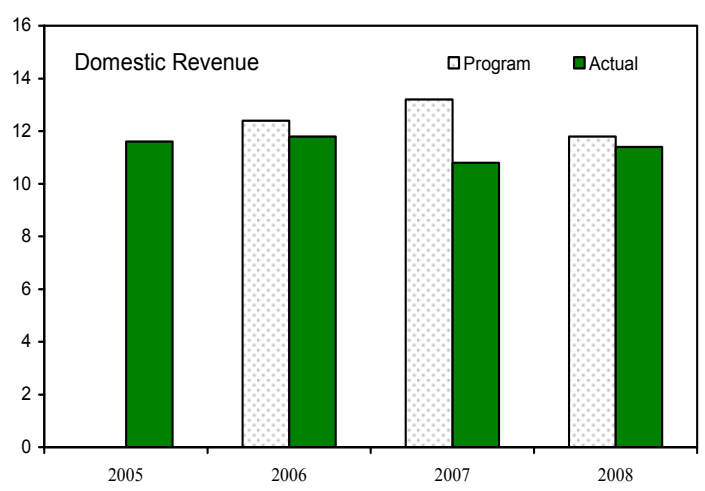

The current account deteriorated in 2008 due to oil and food price shocks, but foreign reserves slightly improved.

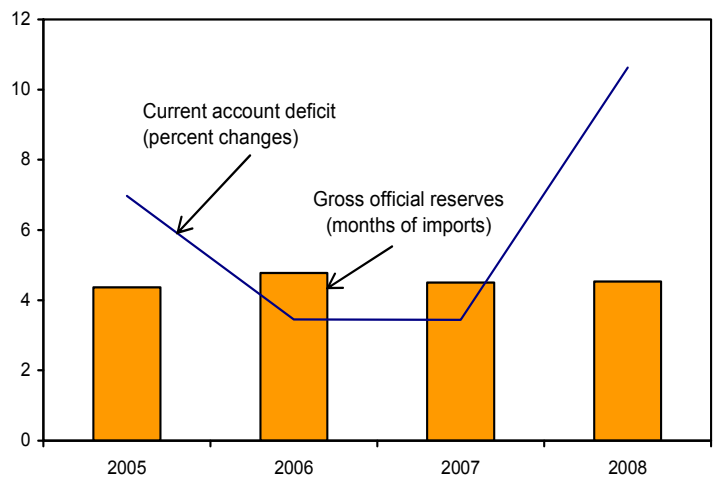

Exogenous shocks kept inflation in double digits in recent years.

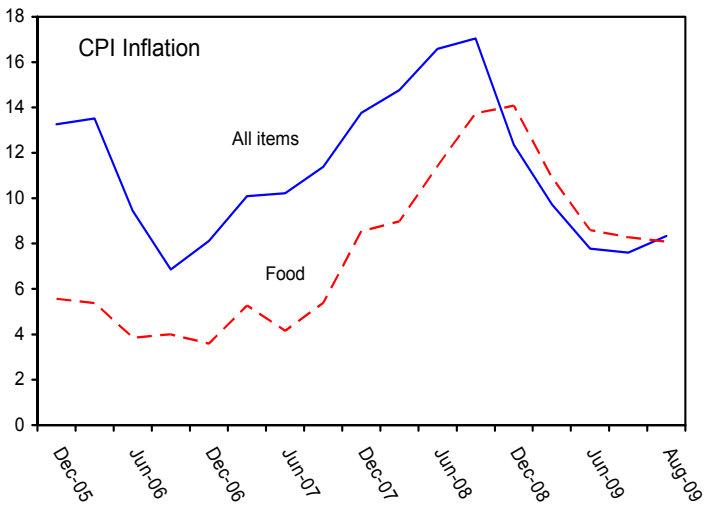

...but cuts in expenditures helped improve the overall fiscal balance.

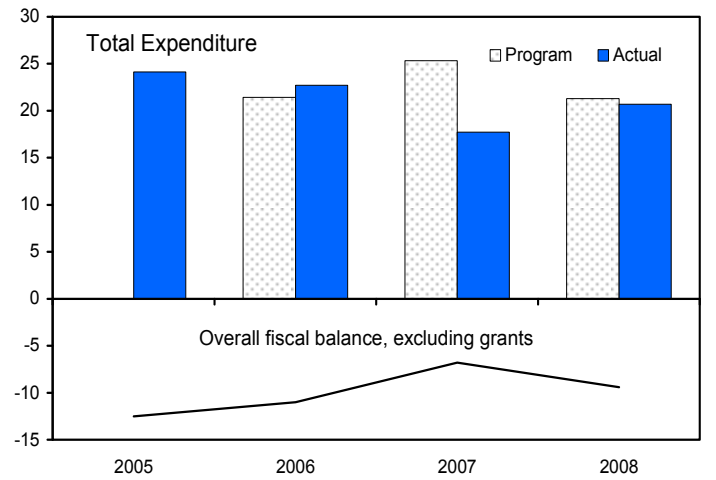

Weakening currency values and price compression in major European trading partners contributed to REER appreciation in 2008. The appreciation is being reversed since early 2009.

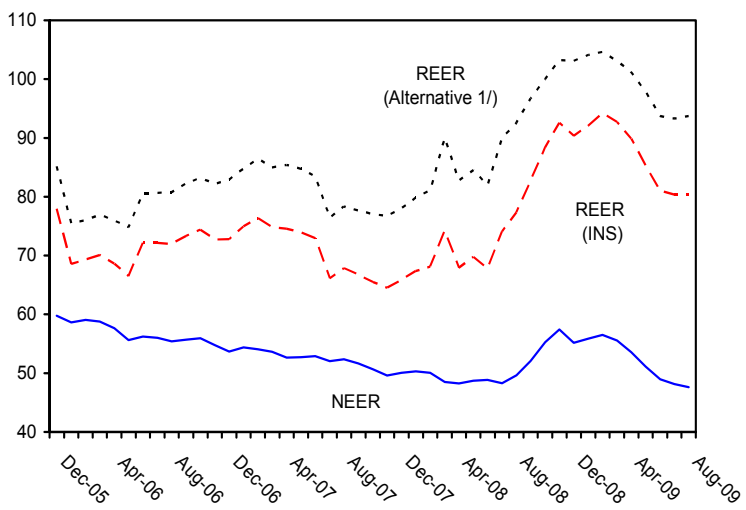

Sources: Sierra Leonean authorities; and IMF staff estimates.

1/ Alternative calculation with weights based on economic importance of the three main currencies (USD, EUR and GBP). 
2. Fiscal performance for the first half of $\mathbf{2 0 0 9}$ was satisfactory. The domestic revenue target was slightly exceeded, as import duties outpaced shortfalls in other revenues.

Expenditure commitments were contained, partly due to delays in an expected disbursement of Le 41 billion ( 0.6 percent of GDP) in external budgetary assistance. The domestic primary fiscal deficit was 1.3 percent of GDP - about 0.5 percent of GDP below the June target (Table 3).

3. Monetary policy remained tight in the first half of 2009. Reserve money grew by about 6 percent through June, about 7 points lower than envisaged, reflecting the better fiscal stance and sales of T-bills for monetary operations by the BSL (Table 4). While annual growth of broad money and credit to the private sector remained high, the pace significantly moderated in the second quarter. The high money growth is partly explained by the new entrance of banks in 2008 that led to more competition and a decline in the currency-to-deposit ratio. After the average interest rate on T-bills was halved in 2008, the rate increased from 9.1 percent at end2008 to 10.8 percent at end-September 2009.

\section{Program implementation in the first half of 2009 was satisfactory (MEFP Table}

1 and 2). All end-June 2009 quantitative PCs were met, though the indicative target on poverty-related spending was missed, partly reflecting lower total budget spending. On the structural targets, the continuous structural benchmark on the monthly meeting of the MPC was met. Although the structural benchmark for adoption by the Cabinet of the implementation decree for the new customs law and regulations was not observed by end-June as envisaged, the measure was implemented in early September 2009. Of the two structural benchmarks for September 2009, the one on adopting a small taxpayer regime was met, and the one on adopting new off-site surveillance guidelines has been postponed because of delays in revising the underlying legislation.

\section{The mission learned that the standard continuous performance criterion on the} introduction or modification of multiple currency practices (MCPs) had not been observed because of the introduction of separate windows in the foreign exchange auction. The BSL introduced a separate window for oil importers in November 2008 and another window for rice importers in August 2009. These separate windows were eliminated in September 2009, following staff representations. Failure to report to staff the introduction of the first window resulted in two noncomplying disbursements at the completion of the third (December 2008) and fourth (June 2009) reviews. A memorandum from the Managing Director to the Executive Board concerning these noncomplying disbursements, and proposing waivers for the non-observance of the PC, has been circulated together with this report. The introduction of the second window violated the continuous performance criterion during the period covered by the fifth program review and the authorities are requesting a waiver for noncompliance with this PC in the attached letter of intent. 


\section{Policy Discussions}

6. Discussions focused on (i) the outlook for 2009 and 2010; (ii) policies to reach the program objectives for the remainder of the year; (iii) the broad parameters of the 2010 budget; and (iv) the structural reform agenda. The policy discussions were largely guided by the PRSP-II that was launched in May 2009.

\section{A. Macroeconomic Framework}

7. The global economic downturn has negatively affected Sierra Leone. The mining sector has suffered from a decline in exports and production, and delays in investment projects. Other sectors have also been affected, notably services, tourism, and construction. On the positive side, output growth has continued to benefit from an improved supply of electricity, initiatives to increase agriculture productivity, and increased public investment in basic infrastructure.

\section{The macroeconomic outlook for 2009 has been revised to reflect recent}

developments. Projected real GDP growth remains at 4 percent in 2009, while annual inflation (end of period) is projected to rise slightly to 9.5 percent, above the 9 percent previously envisaged, mostly because of the currency depreciation in the first half of the year. The recent rebound in mining production and exports and increase in exports of agriculture products (in particular cocoa and coffee) will contribute to reducing the external current account deficit from 10.6 percent of GDP in 2008 to 9.4 percent in 2009. Gross foreign exchange reserves, including the recent SDR allocation, are projected to reach 6.5 months of import coverage year-end.

\section{A more favorable global economic environment is expected to bolster Sierra} Leone's macroeconomic performance in 2010-12. Real GDP growth is projected to recover to 4.7 percent in 2010 and reach 6 percent by 2012. Economic growth will benefit from improved energy supply, higher agricultural productivity, investment in basic infrastructure, and initiatives to improve the business climate. Lower commodity prices and healthy domestic food production should continue to ease inflationary pressures. Inflation is projected to decline to 8 percent in 2010 and 6 percent in 2012. Gross foreign exchange reserves are projected to decline gradually to 5.5 months of imports in 2012 in line with the recovery of imports as the impact of the global downturn disappears. There are, however, significant downside risks to these projections should the global economic recovery be slower than anticipated. 
Sierra Leone: Medium-Term Macroeconomic Indicators, 2008-12

\begin{tabular}{|c|c|c|c|c|c|c|}
\hline & 2008 & 20 & 09 & 2010 & 2011 & 2012 \\
\hline & Est. & Prog. $^{1}$ & Rev. Prog. & & Proj. & \\
\hline Real GDP (annual percentage change) & 5.5 & 4.0 & 4.0 & 4.7 & 5.5 & 6.0 \\
\hline Consumer prices (percentage change; end of period) & 12.2 & 9.0 & 9.5 & 8.0 & 7.0 & 6.0 \\
\hline Consumer prices (percentage change; annual average) & 14.8 & 10.6 & 9.5 & 8.8 & 7.5 & 6.5 \\
\hline Gross domestic investment & 14.7 & 14.8 & 15.1 & 16.5 & 17.6 & 17.8 \\
\hline External current account balance, including official transfers & -10.6 & -8.5 & -9.4 & -8.7 & -7.5 & -7.2 \\
\hline External current account balance, excluding official transfers & -13.1 & -12.3 & -13.2 & -12.4 & -11.2 & -10.7 \\
\hline Gross official reserves (months of imports) & 4.5 & 5.0 & 6.5 & 6.1 & 5.7 & 5.5 \\
\hline
\end{tabular}

${ }^{1}$ IMF Country Report No. 09/215.

\section{B. Policies for 2009 and 2010}

10. Discussions focused on policies to mitigate the impact of the global downturn without undermining macroeconomic stability and growth prospects. Prudent economic management in 2008 and the first half of 2009 has given Sierra Leone the scope to safeguard economic stability and achieve higher economic growth in the medium term. The authorities indicated their continued commitment to sound macroeconomic policies.

\section{Fiscal policy}

11. Fiscal policy in the remainder of 2009 will aim to keep domestic revenue collection on track while containing unanticipated spending pressures. The annual domestic revenue target is expected to be met due to continued good performance of trade-related taxes and the transfer of all off-budget revenues to the CRF (MEFP, ๆ12). However, the budget has been under pressure from unanticipated spending needs and postponement of NPA contributions totaling 0.8 percent of GDP. This reflects additional costs to complete the Bumbuna hydropower plant, an extension of the emergency power project by three months, unbudgeted deployment of Sierra Leonean forces to the UN mission in Darfur, and additional counterpart funds to key road projects. Staff concurred with the authorities that after what had been done earlier in the year there is little scope for cutting spending in other areas without affecting priority spending. While about half of the unanticipated financing gap could be covered by additional grants from the EC, staff agreed that domestic bank financing could cover the remaining 0.4 percent of GDP. The overall fiscal deficit, including grants, has been increased by 0.4 percent of GDP.

\section{The authorities' budget framework for $\mathbf{2 0 1 0}$ aims to maintain economic stability} and sustain growth through improved revenue performance and increased allocation of resources to capital spending (MEFP -18-21). Domestic revenue is projected to rise from 11.3 percent of GDP in 2009 to 11.6 percent of GDP. This improvement reflects the broadening of the tax base and efficiency gains expected from (i) continuing NRA modernization, particularly the completion of ASYCUDA in customs clearing; (ii) the introduction of the GST 
in January 2010; and (iii) the full year effect of the transfer of all off-budget revenue to the $\mathrm{CRF}$, in line with the 2009 Finance Act. On expenditures, the wage bill is projected to remain close to 6 percent of GDP. It assumes a 20 percent increase in basic pay for civil servants and hiring of new teachers and police officers. Current nonwage, noninterest expenditures are projected to decline by 1 percentage point of GDP, mostly due to the savings from discontinuing the emergency power project. Domestically financed capital spending is budgeted to increase by about 1 percent of GDP, in line with the PRSP objective of improving infrastructure, particularly roads and water distribution. The primary deficit is projected to decline by 0.3 percentage points of GDP. Domestic financing will remain at 1.8 percent of GDP, including 0.5 percent of GDP to reduce domestic arrears.

Sierra Leone: Fiscal Indicators, 2008-12

(Percent of GDP, unless otherwise indicated)

\begin{tabular}{|c|c|c|c|c|c|c|}
\hline & \multirow{2}{*}{$\begin{array}{r}2008 \\
\text { Act. }\end{array}$} & \multicolumn{2}{|c|}{2009} & 2010 & 2011 & 2012 \\
\hline & & Prog. ${ }^{1}$ & Rev. Prog. & \multicolumn{3}{|c|}{ Proj. } \\
\hline Revenue and grants & 15.9 & 18.2 & 18.6 & 18.6 & 18.6 & 18.8 \\
\hline Domestic revenue & 11.4 & 11.0 & 11.3 & 11.6 & 12.0 & 12.3 \\
\hline Total expenditure and net lending & 20.7 & 22.2 & 23.0 & 22.8 & 23.6 & 23.2 \\
\hline \multirow{2}{*}{$\begin{array}{l}\text { Of which: current nonwage noninterest expenditure } \\
\text { total capital expenditure }\end{array}$} & 6.8 & 7.1 & 7.6 & 6.6 & 6.7 & 6.5 \\
\hline & 6.2 & 7.4 & 7.7 & 8.1 & 9.0 & 9.0 \\
\hline Domestic primary fiscal balance & -2.4 & -3.3 & -3.8 & -3.5 & -3.3 & -2.7 \\
\hline \multicolumn{7}{|l|}{ Overall fiscal balance, commitment basis } \\
\hline Excluding grants & -9.4 & -11.2 & -11.8 & -11.2 & -11.6 & -11.0 \\
\hline Including grants & -4.8 & -4.0 & -4.4 & -4.2 & -5.0 & -4.4 \\
\hline Domestic financing & 2.7 & 1.7 & 1.8 & 1.8 & 2.1 & 1.4 \\
\hline Of which: bank financing & 3.7 & 1.9 & 2.3 & 2.2 & 2.1 & 1.5 \\
\hline
\end{tabular}

Sources: Sierra Leonean authorities, and IMF staff estimates and projections.

' IMF Country Report No. 09/215.

\section{The authorities recognize the need to accelerate efforts to improve tax} administration and broaden the tax base (MEFP, \13). At 11.3 percent of GDP in 2009, Sierra Leone's domestic revenue is among the lowest in sub-Saharan Africa. Measures to make the NRA more efficient include (i) establishing a domestic tax department, as a vehicle for achieving integration of domestic tax collection; (ii) integrating administration of the GST into the Large Taxpayer Office; (iii) enforcing tax legislation to eliminate discretionary duty waivers and tax exemptions and reduce tax evasion; and (iv) intensifying field audits and enforcement of the payment of tax arrears. An FAD TA mission is scheduled to review GST implementation once the tax has been in effect for a few months.

\section{Monetary and exchange rate policies}

\section{Monetary policy through end-2009 will aim at keeping inflation in the single digits.}

The BSL will continue to conduct open market operations and sales of foreign exchange to mop up excess liquidity. Growth in reserve money for 2009 has been revised down to about 8 percent from 13 percent in the program because of the slower growth in the first half of the year, and the decline in money demand stemming from the depreciation of the leone against US dollar. With technical assistance from MCM, the BSL is working to enhance the flexibility 
of the current liquidity management framework. To this end, the BSL intends to increase its use of newly introduced repo and reverse repo transactions and continue the conversion of noninterest-bearing government securities into marketable securities.

15. The quality of the loan portfolio remains a concern. Although nonperforming loans (NPLs) relative to gross loans halved between March 2008 and June 2009, they remain high at 17.8 percent. $^{2}$ To reduce vulnerability from NPLs, the BSL is enforcing the minimum capital requirement of Le 15 billion for all banks by end-2009. Although Sierra Leone's banks have little exposure to international financial markets, the BSL needs to closely monitor compliance with prudential regulations.

16. The authorities are committed to maintaining a flexible exchange rate to facilitate adjustment to external shocks. The BSL, however, has recently increased its net foreign exchange sales in the auction market to reduce short-term exchange rate volatility caused by a temporary shortfall in net inflows of foreign exchange. As a result, the targeted increase in gross foreign exchange reserves during the second half of 2009 is no longer attainable. Gross reserves are now targeted at US\$205 million by end-December 2009 (about US\$13 million below the original target) or US\$333 million at end-December 2009 (6.5 months of imports), including the new SDR allocation. ${ }^{3}$

17. Even after the removal of the special windows for oil and rice importers in the foreign exchange auction, the applied multiple-price Dutch auction system gives rise to MCP as there is no formal mechanism in place to prevent spreads of effective rates between the winning bids from exceeding two percent. Since the current auction system, i.e., without the special windows, has been in operation since long before the start of the current PRGF arrangement, it does not violate the continuous PC on the introduction or modification of MCPs. However, the BSL will consult with the IMF on the best way to introduce formal mechanisms that would eliminate the MCP by end-June 2010 (MEFP, $\uparrow 23$ ).

18. The stance of monetary policy in $\mathbf{2 0 1 0}$ will be geared towards keeping low inflation. Reserve and broad money are envisaged to increase by 12.5 percent. Given the fiscal projections, there will be room for an 18 percent increase in private sector credit and a small buildup of gross international reserves.

\section{External sector}

19. Sierra Leone's balance of payments outlook is expected to improve over the medium term if the recent rebound in exports continues. Exports are now projected to fall

\footnotetext{
${ }^{2}$ The high NPL ratio is partly explained by a stricter definition of nonperforming loans which includes loans that are overdue by more than 1 month and not 3 months as used in many other countries. Including only loans overdue by more than 3 months, the NPL ratio reduces to 10.5 percent at end-June 2009.

${ }^{3}$ For now, the authorities are comfortable keeping the allocation as a foreign exchange reserve buffer.
} 
by only about 4 percent in 2009 compared to a decline of more than 25 percent previously envisaged. This reflects a recent pick-up in exports of diamonds and agricultural products as world prices and export volumes rebounded. A major diamond mining company has restarted activity and initiatives to boost agricultural exports are generating positive results. On the import side, the value of fuel imports is projected to decline less than programmed because of higher world oil prices. With the expected slow recovery in the global economy, the current account deficit would narrow from 9.4 percent of GDP in 2009 to 8.7 percent of GDP in 2010.

\section{As indicated in the attached LIC-DSA, Sierra Leone is at moderate risk of debt} distress but should pursue a prudent borrowing policy. Under the projected medium- and long-term outlook of exports and assuming continuation of prudent macroeconomic policies, the PV of external debt-to-export ratio is projected to decline from 98 percent in 2009 to 77 percent in 2019 and 60 percent in 2029, thus staying below the threshold of 100 percent during the projection period (2009-29). The PV of external debt-to-GDP ratio would remain in the 15-19 percent range and debt service would stay under 6 percent of exports during the entire projection period, both well below their thresholds (30 and 15 percent, respectively). The LICDSA projects that the domestic debt burden will decline, leading to a gradual drop in the PV of total debt-to-GDP ratio from 34 percent in 2008 to 27 percent in 2029. An alternative scenario, however, underscores the need to keep borrowing policy prudent, since Sierra Leone remains vulnerable to adverse economic developments and external shocks. Staff encouraged the authorities to pursue efforts to get debt relief from commercial creditors under the IDA Debt Reduction Facility ${ }^{4}$ and to contract new external financing only in the form of grants and highly concessional loans.

\section{The government is making good faith efforts to resolve external arrears to} commercial creditors. The authorities continue to engage these creditors and have made goodwill payments to some of them to avoid litigation. There are no new developments in Sierra Leone's relations with its commercial creditors that could undermine program implementation. Ongoing efforts provide sufficient financing assurances for the Fund-supported program.

\section{Structural reforms}

\section{The authorities reiterated their commitment to macro critical structural reforms.} They will focus on (MEFP |23-29):

- Implementing the Financial Sector Development Plan (FSDP) to promote a sound and well-functioning financial system. In parallel, the BSL is working to strengthen

\footnotetext{
${ }^{4}$ In February 2009, the World Bank approved a grant to fund the preparatory work for the operation, including financing for legal and financial advisors and audits. Unreconciled records suggest that the stock of external debt to commercial creditors could be as high as US\$243 million. The buy-back operation is expected to be completed in 2011.
} 
banking supervision and is preparing to establish a credit reference bureau and an efficient payments system.

- Deepening PFM reforms and building capacity for effective delivery of basic public services. A new integrated PFM reform program, consolidating progress made so far, is under implementation. The authorities recognized the need to strengthen the MTEF process to ensure consistency with the medium-term objectives of the poverty reduction strategy.

- $\quad$ Restructuring the National Power Authority (NPA) and improving its finances. A study is underway to inform a comprehensive electricity tariff policy to be adopted by December 2009. A financial controller and a commercial manager have been recruited to improve oversight of NPA financial operations.

- Establishing a transparent and automatic pricing framework for petroleum products. The authorities intend to ensure a full pass-through of international prices to the pump prices and avoid discretionary adjustments in the pricing formula to achieve a targeted pump price. The existing Strategic Petroleum Fund (SPF) will be brought under the budget and its purpose clarified.

- Tackling corruption and enhancing transparency in the use of public resources. The power of the Anti-Corruption Commission (ACC) has been strengthened and it will continue to focus on education, outreach activities, and prosecution of corruption cases.

23. Structural reforms in the remainder of the program period will concentrate on measures that will enhance revenue collection and strengthen public expenditure management. To this end, the authorities are taking actions to establish a domestic tax department, integrate the GST administration within the LTO, and recruit and assign budget officers to key MDAs by end-December 2009. Preparations are also being made to introduce the GST on January 1, 2010.

\section{Program Risks ANd Monitoring ANd CAPACITY TO RePAY The Fund}

\section{The major program risks arise from uncertainty about the recovery from the} global economic crisis. A protracted global recovery could lower economic growth and put additional strains on public finances. Also, donors might revisit their aid commitments. The relatively good performance under the current PRGF arrangement confirms the authorities' resolve to follow prudent macroeconomic policies that could mitigate these risks.

25. The revised program modifies four quantitative PCs for end-December. The PCs on net domestic credit to government, net domestic assets, domestic primary fiscal balance, and gross foreign exchange reserves have been modified in line with the revised macroeconomic projections. The PCs and structural benchmarks for end-December are presented in Table 1 and Table 2 of the MEFP. The review of structural policies will be based on assessment of progress on the authorities' reform program, particularly where benchmarks have been set. 
26. Sierra Leone's capacity to repay the Fund is sound (Table 6). The debt service to the Fund will peak in 2015 at 3 percent of exports of goods and services.

27. On Sierra Leone's future relations with the Fund following the expiration of the current PRGF arrangement in May 2010, the authorities have expressed interest in a successor Fund-supported program, which could be negotiated at the time of the sixth review.

\section{StafF ApPraisal}

28. After a period of slowing economic activity, the outlook for Sierra Leone is beginning to improve. Since late 2008 , economic activity has been negatively impacted by slowing global demand and declining inflows of remittances and foreign direct investments. With the currency depreciation and the recent improvement in the external environmentincluding partial recovery of diamond prices and an improved outlook for agricultural exports - exports and economic activity are expected to begin to turn around. In 2010, growth is expected to increase and inflation to remain in single digits.

29. Improving revenue collection and enhancing budget execution will be critical. Because revenue collection is low by regional standards, the authorities need to step up the efforts to strengthen tax administration and broaden the tax base. The GST launch planned for January 2010 is an important step in this direction. Weaknesses in budget execution need to be addressed. The authorities are encouraged to accelerate efforts to improve public expenditure management to ensure greater transparency, accountability, and efficiency in the use of public resources.

30. Staff supports the monetary policy aim at keeping inflation in single digits. The BSL must remain watchful for a possible re-emergence of inflationary pressures in case the leone continues to depreciate. It may also wish to explore options to expand its short-term monetary instruments to increase flexibility in the conduct of monetary policy.

31. Vigilance against financial sector risks is crucial. Although the banking sector has coped well thus far with the global financial crisis, the BSL should closely monitor bank open positions in foreign currencies and compliance with prudential regulations. Notwithstanding the recent decline, the high level of NPLs remains a cause for concern.

32. Staff welcomes the authorities' continued commitments to structural reforms. The focus is appropriately on deepening the financial sector, reforming the electricity sector to provide reliable and cost-efficient service throughout the country, enhancing efficiency in the use of public resources, promoting good governance, and establishing a transparent and automatic pricing framework for petroleum products.

33. Based on performance to date and Sierra Leone's continued commitment to program implementation and the consolidation of the foreign exchange auction system into a single competitive window, staff supports the requested waiver for nonobservance of the continuous PC on the introduction or modification of MCPs and recommends completion of the fifth PRGF review and the financing assurances review. Staff also supports the modification of the quantitative PCs for end-December 2009 in view of the authorities' continued commitment to sound macroeconomic policies and to implementing their structural reform agenda. 
Table 1. Sierra Leone: Selected Economic and Financial Indicators, 2007-12

\begin{tabular}{|c|c|c|c|c|c|c|c|}
\hline & \multirow[t]{2}{*}{2007} & \multirow{2}{*}{$\begin{array}{r}2008 \\
\text { Est. }\end{array}$} & \multicolumn{2}{|c|}{2009} & 2010 & 2011 & 2012 \\
\hline & & & Prog. ${ }^{1}$ & Rev. Prog. & \multicolumn{3}{|c|}{ Proj. } \\
\hline & \multicolumn{7}{|c|}{ (Annual percentage change, unless otherwise indicated) } \\
\hline \multicolumn{8}{|l|}{ Income and prices } \\
\hline Real GDP & 6.4 & 5.5 & 4.0 & 4.0 & 4.7 & 5.5 & 6.0 \\
\hline GDP deflator & 10.8 & 11.2 & 8.8 & 6.3 & 7.5 & 6.3 & 5.5 \\
\hline Nominal GDP & 17.9 & 17.4 & 13.1 & 10.5 & 12.5 & 12.1 & 11.9 \\
\hline Consumer prices (end of period) & 13.8 & 12.2 & 9.0 & 9.5 & 8.0 & 7.0 & 6.0 \\
\hline Consumer prices (annual average) & 11.7 & 14.8 & 10.6 & 9.5 & 8.8 & 7.5 & 6.5 \\
\hline \multicolumn{8}{|l|}{ Money and credit } \\
\hline Broad money & 25.9 & 26.4 & 16.9 & 12.9 & 12.5 & 12.1 & 10.5 \\
\hline Velocity (level) & 4.4 & 4.1 & 4.0 & 4.0 & 4.0 & 4.0 & 4.1 \\
\hline Domestic credit $^{2}$ & 27.2 & 65.5 & 26.2 & 29.0 & 20.6 & 6.6 & 6.0 \\
\hline Government $^{2}$ & 17.6 & 71.9 & 35.5 & 26.6 & 23.5 & 5.3 & 5.6 \\
\hline Private sector & 39.4 & 56.8 & 15.5 & 31.8 & 18.0 & 8.5 & 6.6 \\
\hline Reserve money & 26.0 & 10.2 & 13.2 & 7.8 & 12.5 & 10.4 & 8.8 \\
\hline Interest rate ${ }^{3}$ & 21.3 & 9.1 & $\ldots$ & $\ldots$ & $\ldots$ & $\ldots$ & $\ldots$ \\
\hline \multicolumn{8}{|l|}{ External sector } \\
\hline Exports (US\$) & 2.9 & -9.2 & -26.6 & -3.8 & 19.4 & 17.3 & 10.2 \\
\hline Imports (US\$) & 2.2 & 24.7 & -10.9 & -3.5 & 11.7 & 9.7 & 8.1 \\
\hline Export volumes & -6.0 & -23.2 & -8.6 & 13.1 & 8.6 & 13.1 & 6.9 \\
\hline Import volumes & 0.2 & 7.4 & 3.6 & 7.5 & 3.5 & 7.1 & 6.4 \\
\hline Terms of trade $(-=$ deterioration $)$ & -1.9 & -2.9 & -4.1 & -6.7 & 2.4 & 1.4 & 1.6 \\
\hline Real effective exchange rate $(-=$ depreciation; eop & -1.0 & 9.1 & $\ldots$ & $\ldots$ & $\ldots$ & $\ldots$ & $\ldots$ \\
\hline & \multicolumn{7}{|c|}{ (Percent of GDP) } \\
\hline Saving and Investment & & & & & & & \\
\hline Gross national saving & 9.7 & 4.1 & 6.2 & 5.7 & 7.8 & 10.1 & 10.6 \\
\hline Gross domestic saving & 6.1 & 1.7 & 3.2 & 1.8 & 2.7 & 4.5 & 5.8 \\
\hline Central government & 0.3 & -0.6 & -1.6 & -1.9 & -0.6 & -0.3 & 0.3 \\
\hline Private sector & 5.8 & 2.3 & 4.8 & 3.7 & 3.3 & 4.8 & 5.5 \\
\hline Gross domestic investment & 13.2 & 14.7 & 14.8 & 15.1 & 16.5 & 17.6 & 17.8 \\
\hline Government & 3.5 & 6.2 & 7.4 & 7.7 & 8.1 & 9.0 & 9.0 \\
\hline Private & 9.7 & 8.6 & 7.4 & 7.5 & 8.4 & 8.6 & 8.8 \\
\hline \multicolumn{8}{|l|}{ External sector } \\
\hline Current account, including official transfers & -3.4 & -10.6 & -8.5 & -9.4 & -8.7 & -7.5 & -7.2 \\
\hline Current account, excluding official transfers & -7.0 & -13.1 & -12.3 & -13.2 & -12.4 & -11.2 & -10.7 \\
\hline Overall balance of payments & 1.9 & -1.2 & -1.4 & 4.5 & -0.2 & 0.5 & 0.7 \\
\hline \multicolumn{8}{|l|}{ Central government } \\
\hline Domestic revenue & 10.8 & 11.4 & 11.0 & 11.3 & 11.6 & 12.0 & 12.3 \\
\hline Total expenditure and net lending & 17.7 & 20.7 & 22.2 & 23.0 & 22.8 & 23.6 & 23.2 \\
\hline Of which: current expenditure & 13.3 & 14.6 & 14.8 & 15.3 & 14.7 & 14.6 & 14.2 \\
\hline Overall fiscal balance & 25.2 & -4.8 & -4.0 & -4.4 & -4.2 & -5.0 & -4.4 \\
\hline (Excluding grants and MDRI relief) & -6.8 & -9.4 & -11.2 & -11.8 & -11.2 & -11.6 & -11.0 \\
\hline Domestic primary fiscal balance ${ }^{5}$ & -1.9 & -2.4 & -3.3 & -3.8 & -3.5 & -3.3 & -2.7 \\
\hline Domestic financing & 2.2 & 2.7 & 1.7 & 1.8 & 1.8 & 2.1 & 1.4 \\
\hline
\end{tabular}

(Percent of exports and nonfactor services, unless otherwise indicated)

\begin{tabular}{|c|c|c|c|c|c|c|c|}
\hline Debt service due after debt relief, incl. to $\mathrm{IMF}^{6}$ & 4.3 & 1.9 & 3.9 & 3.3 & 3.7 & 4.5 & 3.8 \\
\hline Present value of debt-to-exports ratio ${ }^{7}$ & 40.8 & 71.9 & 82.2 & 97.5 & 89.0 & 85.2 & 89.0 \\
\hline \multirow[t]{2}{*}{ Present value of debt-to-GDP ratio ${ }^{7}$} & 8.6 & 11.8 & 9.2 & 15.3 & 16.0 & 16.2 & 17.1 \\
\hline & \multicolumn{7}{|c|}{ (US\$ million, unless otherwise indicated) } \\
\hline $\begin{array}{l}\text { External current account, excluding } \\
\text { official transfers } \\
\text { Gross international reserves }\end{array}$ & $\begin{array}{r}-115.8 \\
215.5\end{array}$ & $\begin{array}{r}-255.9 \\
209.5\end{array}$ & $\begin{array}{r}-257.0 \\
218.0\end{array}$ & $\begin{array}{r}-255.5 \\
333.5\end{array}$ & $\begin{array}{r}-245.6 \\
338.5\end{array}$ & $\begin{array}{r}-242.6 \\
343.5\end{array}$ & $\begin{array}{r}-252.0 \\
353.5\end{array}$ \\
\hline (Months of imports) ${ }^{8}$ & 4.5 & 4.5 & 5.0 & 6.5 & 6.1 & 5.7 & 5.5 \\
\hline GDP & $1,663.7$ & $1,954.3$ & $2,091.1$ & $1,939.2$ & $1,982.8$ & $2,158.7$ & $2,344.2$ \\
\hline GDP (Le billions) & $4,966.5$ & $5,828.3$ & $6,586.9$ & $6,442.2$ & $7,247.1$ & $8,126.9$ & $9,090.0$ \\
\hline
\end{tabular}

Sources: Sierra Leonean authorities; and IMF staff estimates and projections.

${ }^{1}$ IMF Country Report No. 09/215.

${ }^{2}$ The numbers reflect the impact of the MDRI.

${ }^{3} 91$-day treasury bill rate (end of period).

${ }^{4}$ For 2007, MDRI relief from IDA and AfDF (both as stock of debt relief).

${ }^{5}$ Domestic revenue minus total expenditure and net lending, excluding interest payments, and externally financed capital expenditure.

${ }^{6}$ Percent of exports of goods and services.

'The figure for 2007 is taken from the June 2008 DSA. The estimate for 2008 and the projections for 2009-12 are derived from the 2009 DSA. The revisions to the debt ratios, compared to those projected in the June 2009 staff report, are mostly due to higher future debt service payments and a lower discount rate.

${ }^{8}$ Months of imports of goods and services of subsequent year. 
Table 2. Sierra Leone: Central Government Financial Operations, 2008-12 (Le Billions, unless otherwise indicated)

\begin{tabular}{|c|c|c|c|c|c|c|c|c|}
\hline & \multirow[t]{3}{*}{2008} & \multicolumn{4}{|c|}{2009} & \multirow[t]{3}{*}{2010} & \multirow{3}{*}{$\frac{2011}{\text { Proj. }}$} & \multirow[t]{3}{*}{2012} \\
\hline & & \multicolumn{2}{|c|}{ June } & \multicolumn{2}{|c|}{ Full year } & & & \\
\hline & & Prog. ${ }^{1}$ & Est. & Prog. ${ }^{1}$ & Rev. Prog. & & & \\
\hline Total revenue and grants & 927 & 574 & 536 & 1,199 & 1,197 & 1,348 & 1,515 & 1,709 \\
\hline Domestic revenue & 662 & 343 & 347 & 725 & 725 & 844 & 973 & 1,114 \\
\hline Tax on income and profits & 196 & 101 & 100 & 215 & 209 & 241 & 273 & 311 \\
\hline Domestic taxes on goods and services & 154 & 97 & 83 & 214 & 186 & 219 & 245 & 272 \\
\hline Of which: excise taxes on petroleum products & 70 & 51 & 42 & 112 & 91 & 115 & 122 & 133 \\
\hline Import duties & 279 & 130 & 153 & 265 & 296 & 342 & 413 & 484 \\
\hline Nontax revenue & 34 & 15 & 11 & 30 & 33 & 42 & 42 & 47 \\
\hline Grants & 265 & 231 & 189 & 475 & 472 & 505 & 542 & 595 \\
\hline Program & 144 & 119 & 87 & 250 & 243 & 268 & 270 & 290 \\
\hline Of which: HIPC assistance & 13 & 3 & 4 & 13 & 13 & 13 & 15 & 14 \\
\hline Peace Building Fund & 15 & 0 & 9 & 12 & 9 & $\ldots$ & $\ldots$ & $\ldots$ \\
\hline Projects & 121 & 112 & 102 & 225 & 229 & 236 & 272 & 305 \\
\hline MDRI assistance from IDA and AfDF & $\ldots$ & $\ldots$ & & & $\ldots$ & $\ldots$ & $\ldots$ & $\ldots$ \\
\hline Total expenditure and net lending & 1,207 & 722 & 624 & 1,463 & 1,483 & 1,655 & 1,918 & 2,110 \\
\hline Current expenditure & 848 & 478 & 445 & 977 & 988 & 1,067 & 1,187 & 1,292 \\
\hline Wages and salaries & 334 & 192 & 196 & 400 & 400 & 453 & 508 & 567 \\
\hline Current noninterest, nonwage expenditure & 393 & 239 & 208 & 461 & 487 & 477 & 543 & 580 \\
\hline Goods and services & 285 & 167 & 142 & 312 & 332 & 306 & 349 & 364 \\
\hline Of which : Emergency Power Project & 83 & 35 & 35 & 52 & 69 & $\ldots$ & $\ldots$ & $\ldots$ \\
\hline Transfers to local councils & 27 & 29 & 24 & 60 & 60 & 70 & 77 & 86 \\
\hline Grants to educational institutions & 29 & 15 & 16 & 32 & 39 & 40 & 50 & 56 \\
\hline Transfers to Road Fund & 46 & 27 & 25 & 56 & 54 & 57 & 65 & 74 \\
\hline Elections & 6 & 0 & 0 & 0 & 1 & 3 & 1 & 0 \\
\hline Interest payments & 120 & 47 & 41 & 116 & 101 & 136 & 136 & 144 \\
\hline Domestic & 109 & 42 & 36 & 104 & $87^{\top}$ & $120^{7}$ & 124 & 133 \\
\hline Foreign & 12 & 5 & 5 & 12 & 15 & 16 & 12 & 12 \\
\hline Capital expenditure and net lending & 359 & 245 & 179 & 486 & 495 & 588 & 731 & 818 \\
\hline Capital expenditure & 359 & 245 & 179 & 487 & 495 & 588 & 731 & 818 \\
\hline Externally financed & 284 & 211 & 155 & 406 & 410 & 422 & 544 & 609 \\
\hline Domestically financed & 75 & 34 & 24 & 81 & 85 & 166 & 187 & 209 \\
\hline Net lending & 0 & 0 & 0 & -1 & 0 & 0 & 0 & 0 \\
\hline \multicolumn{9}{|l|}{ Overall balance (commitment basis) } \\
\hline Excluding grants & -545 & -379 & -277 & -738 & -758 & -811 & -946 & -996 \\
\hline Including grants & -281 & -148 & -88 & -263 & -286 & -306 & -403 & -401 \\
\hline Total financing & 281 & 148 & 88 & 263 & 286 & 306 & 403 & 401 \\
\hline Foreign & 143 & 90 & 39 & 155 & 171 & 173 & 229 & 269 \\
\hline Borrowing & 163 & 100 & 53 & 181 & 214 & 222 & 310 & 343 \\
\hline Project & 163 & 100 & 53 & 181 & 181 & 185 & 272 & 305 \\
\hline Program & 0 & 0 & 0 & 0 & 33 & 37 & 38 & 39 \\
\hline Amortization & -20 & -10 & -14 & -27 & -43 & -49 & -81 & -74 \\
\hline Domestic & 158 & 71 & 40 & 109 & 115 & 134 & 175 & 132 \\
\hline Bank financing & 218 & 71 & 55 & 123 & 151 & 157 & 171 & 137 \\
\hline Central bank & 120 & 64 & 64 & 100 & 129 & 124 & 127 & 89 \\
\hline Of whic $\mathrm{h}$ : change in MDRI-related deposits & 118 & 64 & 23 & 100 & 99 & 124 & 127 & 89 \\
\hline Commercial banks & 98 & $8^{r}$ & -9 & 23 & 23 & 33 & 44 & 48 \\
\hline Nonbank financing & -60 & -1 & -15 & -14 & -36 & -23 & 4 & -6 \\
\hline Claims on gov. by nonbank sector & -34 & -5 & -10 & 7 & 8 & 11 & 34 & 25 \\
\hline Privatization and other receipts & 4 & 12 & 10 & 24 & 10 & 0 & 0 & 0 \\
\hline Change in arrears & -46 & -30 & -38 & -45 & -54 & -34 & -30 & -31 \\
\hline Float (checks payable and outstanding commitme & 16 & 22 & 23 & 0 & 0 & 0 & 0 & 0 \\
\hline Unaccounted ( $-=$ overfinancing of the budget) & -21 & -12 & 9 & 0 & 0 & 0 & 0 & 0 \\
\hline \multicolumn{9}{|l|}{ Memorandum items } \\
\hline Total poverty expenditures & 330 & 179 & 138 & 337 & 337 & 402 & 469 & 549 \\
\hline Domestic primary balance $^{2}$ & -141 & -121 & -81 & -219 & -247 & -253 & -265 & -242 \\
\hline Total wages and salaries (percent of domestic revenue) & 50 & 56 & 57 & 55 & 55 & 54 & 52 & 51 \\
\hline
\end{tabular}

Sources: Sierra Leonean authorities, and IMF staff estimates.

${ }^{1}$ IMF Country Report No. 09/215.

${ }^{2}$ Domestic revenue minus total expenditure and net lending, excluding interest payments and externally financed capital expenditures. 
Table 3. Sierra Leone: Central Government Financial Operations, 2008-12 (Percent of GDP)

\begin{tabular}{|c|c|c|c|c|c|c|c|c|}
\hline & \multirow[t]{3}{*}{2008} & \multicolumn{4}{|c|}{2009} & \multirow[t]{3}{*}{2010} & \multirow{3}{*}{$\begin{array}{c}2011 \\
\text { Proj. }\end{array}$} & \multirow[t]{3}{*}{2012} \\
\hline & & \multicolumn{2}{|c|}{ June } & \multicolumn{2}{|c|}{ Full year } & & & \\
\hline & & Prog. ${ }^{1}$ & Est. & Prog. ${ }^{1}$ & Rev. Prog. & & & \\
\hline Total revenue and grants & 15.9 & 8.7 & 8.3 & 18.2 & 18.6 & 18.6 & 18.6 & 18.8 \\
\hline Domestic revenue & 11.4 & 5.2 & 5.4 & 11.0 & 11.3 & 11.6 & 12.0 & 12.3 \\
\hline Tax on income and profits & 3.4 & 1.5 & 1.5 & 3.3 & 3.3 & 3.3 & 3.4 & 3.4 \\
\hline Domestic taxes on goods and services & 2.6 & 1.5 & 1.3 & 3.1 & 2.9 & 3.0 & 3.0 & 3.0 \\
\hline Of which : excise taxes on petroleum products & 1.2 & 0.8 & 0.6 & 1.7 & 1.4 & 1.6 & 1.5 & 1.5 \\
\hline Import duties & 4.8 & 2.0 & 2.4 & 4.1 & 4.6 & 4.7 & 5.1 & 5.3 \\
\hline Nontax revenue & 0.6 & 0.2 & 0.2 & 0.5 & 0.5 & 0.6 & 0.5 & 0.5 \\
\hline Grants & 4.5 & 3.5 & 2.9 & 7.2 & 7.3 & 7.0 & 6.7 & 6.5 \\
\hline Program & 2.5 & 1.8 & 1.4 & 3.8 & 3.8 & 3.7 & 3.3 & 3.2 \\
\hline Of which: HIPC assistance & 0.2 & 0.1 & 0.1 & 0.2 & 0.2 & 0.2 & 0.2 & 0.2 \\
\hline Peace Building Fund & 0.3 & 0.0 & 0.1 & 0.2 & 0.1 & 0.0 & 0.0 & 0.0 \\
\hline Projects & 2.1 & 1.7 & 1.6 & 3.4 & 3.6 & 3.3 & 3.4 & 3.4 \\
\hline MDRI assistance from IDA and AfDF & 0.0 & 0.0 & 0.0 & 0.0 & 0.0 & 0.0 & 0.0 & 0.0 \\
\hline Total expenditure and net lending & 20.7 & 11.0 & 9.7 & 22.2 & 23.0 & 22.8 & 23.6 & 23.2 \\
\hline Current expenditure & 14.6 & 7.2 & 6.9 & 14.8 & 15.3 & 14.7 & 14.6 & 14.2 \\
\hline Wages and salaries & 5.7 & 2.9 & 3.0 & 6.1 & 6.2 & 6.3 & 6.3 & 6.2 \\
\hline Current noninterest, nonwage expenditure & 6.8 & 3.6 & 3.2 & 7.1 & 7.6 & 6.6 & 6.7 & 6.4 \\
\hline Goods and services & 4.9 & 2.5 & 2.2 & 4.7 & 5.2 & 4.2 & 4.3 & 4.0 \\
\hline Of which : Emergency Power Project & 1.4 & 0.5 & 0.5 & 0.8 & 1.1 & 0.0 & 0.0 & 0.0 \\
\hline Transfers to local councils & 0.5 & 0.4 & 0.4 & 0.9 & 0.9 & 1.0 & 0.9 & 0.9 \\
\hline Grants to educational institutions & 0.5 & 0.2 & 0.2 & 0.5 & 0.6 & 0.5 & 0.6 & 0.6 \\
\hline Transfers to Road Fund & 0.8 & 0.4 & 0.4 & 0.9 & 0.8 & 0.8 & 0.8 & 0.8 \\
\hline Elections & 0.1 & 0.0 & 0.0 & 0.0 & 0.0 & 0.0 & 0.0 & 0.0 \\
\hline Interest payments & 2.1 & 0.7 & 0.6 & 1.8 & 1.6 & 1.9 & 1.7 & 1.6 \\
\hline Domestic & 1.9 & 0.6 & 0.6 & 1.6 & 1.3 & 1.7 & 1.5 & 1.5 \\
\hline Foreign & 0.2 & 0.1 & 0.1 & 0.2 & 0.2 & 0.2 & 0.1 & 0.1 \\
\hline Capital expenditure and net lending & 6.2 & 3.7 & 2.8 & 7.4 & 7.7 & 8.1 & 9.0 & 9.0 \\
\hline Capital expenditure & 6.2 & 3.7 & 2.8 & 7.4 & 7.7 & 8.1 & 9.0 & 9.0 \\
\hline Externally financed & 4.9 & 3.2 & 2.4 & 6.2 & 6.4 & 5.8 & 6.7 & 6.7 \\
\hline Domestically financed & 1.3 & 0.5 & 0.4 & 1.2 & 1.3 & 2.3 & 2.3 & 2.3 \\
\hline Net lending & 0.0 & 0.0 & 0.0 & 0.0 & 0.0 & 0.0 & 0.0 & 0.0 \\
\hline \multicolumn{9}{|l|}{ Overall balance (commitment basis) } \\
\hline Excluding grants & -9.4 & -5.8 & -4.3 & -11.2 & -11.8 & -11.2 & -11.6 & -11.0 \\
\hline Including grants & -4.8 & -2.2 & -1.4 & -4.0 & -4.4 & -4.2 & -5.0 & -4.4 \\
\hline Total financing & 4.8 & 2.2 & 1.4 & 4.0 & 4.4 & 4.2 & 5.0 & 4.4 \\
\hline Foreign & 2.5 & 1.4 & 0.6 & 2.3 & 2.7 & 2.4 & 2.8 & 3.0 \\
\hline Borrowing & 2.8 & 1.5 & 0.8 & 2.7 & 3.3 & 3.1 & 3.8 & 3.8 \\
\hline Project & 2.8 & 1.5 & 0.8 & 2.7 & 2.8 & 2.6 & 3.4 & 3.4 \\
\hline Program & 0.0 & 0.0 & 0.0 & 0.0 & 0.5 & 0.5 & 0.5 & 0.4 \\
\hline Amortization & -0.3 & -0.2 & -0.2 & -0.4 & -0.7 & -0.7 & -1.0 & -0.8 \\
\hline Domestic & 2.7 & 1.1 & 0.6 & 1.7 & 1.8 & 1.8 & 2.1 & 1.4 \\
\hline Bank financing & 3.7 & 1.1 & 0.9 & 1.9 & 2.3 & 2.2 & 2.1 & 1.5 \\
\hline Central bank & 2.1 & 1.0 & 1.0 & 1.5 & 2.0 & 1.7 & 1.6 & 1.0 \\
\hline Of whic h: change in MDRI-related deposits & 2.0 & 1.0 & 0.4 & 1.5 & 1.5 & 1.7 & 1.6 & 1.0 \\
\hline Commercial banks & 1.7 & 0.1 & -0.1 & 0.3 & 0.4 & 0.5 & 0.5 & 0.5 \\
\hline Nonbank financing & -1.0 & 0.0 & -0.2 & -0.2 & -0.6 & -0.3 & 0.0 & -0.1 \\
\hline Claims on gov. by nonbank sector & -0.6 & -0.1 & -0.2 & 0.1 & 0.1 & 0.2 & 0.4 & 0.3 \\
\hline Privatization and other receipts & 0.1 & 0.2 & 0.2 & 0.4 & 0.2 & 0.0 & 0.0 & 0.0 \\
\hline Change in arrears & -0.8 & -0.5 & -0.6 & -0.7 & -0.8 & -0.5 & -0.4 & -0.3 \\
\hline Float (checks payable and outstanding commitm€ & 0.3 & 0.3 & 0.4 & 0.0 & 0.0 & 0.0 & 0.0 & 0.0 \\
\hline Unaccounted (- = overfinancing of the budget) & -0.4 & -0.2 & 0.1 & 0.0 & 0.0 & 0.0 & 0.0 & 0.0 \\
\hline
\end{tabular}

Memorandum items

(In percent of GDP, unless otherwise indicated)

Total poverty expenditures

Domestic primary balance

Nominal GDP (Le billions)

5.7
-2.4
5,828

2.7
-1
-8

6,587

$\begin{array}{rr}2.1 & 5.1 \\ -1.3 & -3.3 \\ 6,442 & 6,521\end{array}$

5.2
-3.8
6,442

5.6
-3.5

7,247 $\begin{array}{rr}5.8 & 6.0 \\ -3.3 & -2.7 \\ 8,127 & 9,090\end{array}$

Sources: Sierra Leonean authorities, and IMF staff estimates.

1 IMF Country Report No. 09/215. 
Table 4. Sierra Leone: Monetary Accounts, 2007-10

(Le billions; at actual exchange rates unless otherwise indicated)

\begin{tabular}{|c|c|c|c|c|c|c|c|c|c|c|}
\hline & \multirow[t]{3}{*}{2007} & \multirow[t]{3}{*}{2008} & \multicolumn{7}{|c|}{2009} & \multirow{3}{*}{$\begin{array}{l}2010 \\
\text { Dec } \\
\text { Proj }\end{array}$} \\
\hline & & & \multirow{2}{*}{$\begin{array}{l}\text { Mar. } \\
\text { Act. }\end{array}$} & \multicolumn{2}{|c|}{ June } & \multicolumn{2}{|c|}{ Sept. } & \multicolumn{2}{|c|}{ Dec. } & \\
\hline & & & & Prog. ${ }^{1}$ & Act. & Prog. ${ }^{1}$ & Proj. & Prog. $^{1}$ & Rev. Prog. & \\
\hline & \multicolumn{10}{|c|}{ I. Monetary survey } \\
\hline Net foreign assets & 883 & 832 & 802 & 742 & 807 & 655 & 1,447 & 725 & 1,303 & 1,261 \\
\hline $\begin{array}{l}\text { Net domestic assets } \\
\text { Domestic credit }\end{array}$ & $\begin{array}{l}246 \\
579\end{array}$ & $\begin{array}{l}594 \\
959\end{array}$ & $\begin{array}{r}675 \\
1,020\end{array}$ & $\begin{array}{r}654 \\
1,020\end{array}$ & $\begin{array}{r}715 \\
1,093\end{array}$ & $\begin{array}{r}854 \\
1,219\end{array}$ & $\begin{array}{r}124 \\
1,098\end{array}$ & $\begin{array}{r}846 \\
1,210\end{array}$ & $\begin{array}{r}307 \\
1,237\end{array}$ & $\begin{array}{r}551 \\
1,492\end{array}$ \\
\hline Net credit to government & 307 & 528 & 517 & 649 & 572 & 777 & 538 & 716 & 668 & 825 \\
\hline Of which : For budget financing & -229 & -3 & -15 & 78 & 52 & 121 & 18 & 119 & 148 & 305 \\
\hline Claims on nonfinancial public enterprises & 8 & 17 & 22 & 17 & 24 & 17 & 24 & 17 & 24 & 24 \\
\hline Claims on private sector & 264 & 413 & 481 & 354 & 497 & 425 & 536 & 477 & 545 & 643 \\
\hline Other items (net) & -334 & -365 & -345 & -366 & -378 & -366 & -974 & -365 & -930 & -941 \\
\hline Broad money & 1,128 & 1,426 & 1,477 & 1,451 & 1,522 & 1,565 & 1,571 & 1,668 & 1,611 & 1,812 \\
\hline Money & 550 & 667 & 682 & 759 & 662 & 818 & 684 & 872 & 733 & 825 \\
\hline \multirow[t]{2}{*}{ Quasi money } & 579 & 759 & 795 & 692 & 860 & 746 & 887 & 795 & 878 & 987 \\
\hline & \multicolumn{10}{|c|}{ II. Bank of Sierra Leone } \\
\hline Net foreign assets & 536 & 479 & 475 & 408 & 439 & 327 & 966 & 384 & 884 & 907 \\
\hline Net domestic assets & -112 & -12 & -20 & 81 & 19 & 203 & -472 & 144 & -381 & -341 \\
\hline Net credit to government & 75 & 136 & 107 & 240 & 170 & 361 & 184 & 301 & 260 & 337 \\
\hline For budget financing & -448 & -320 & -343 & -257 & -257 & -220 & -297 & -220 & -192 & -68 \\
\hline For monetary operations & 523 & 457 & 450 & 497 & 426 & 582 & 481 & 521 & 452 & 405 \\
\hline Claims on non financial public enterprises & 0 & 0 & 0 & 0 & 0 & 0 & 0 & 0 & 0 & 0 \\
\hline Claims on private sector & 3 & 7 & 8 & 7 & 10 & 7 & 10 & 7 & 10 & 10 \\
\hline Claims on deposit money banks & 1 & 4 & 4 & 4 & 0 & 4 & 0 & 4 & 0 & 0 \\
\hline Other items (net) & -191 & -160 & -139 & -170 & -161 & -169 & -666 & -168 & -651 & -688 \\
\hline Reserve money & 424 & 467 & 455 & 489 & 458 & 531 & 494 & 529 & 503 & 566 \\
\hline Currency outside banks & 310 & 340 & 335 & 334 & 323 & 331 & 359 & 416 & 364 & 378 \\
\hline Reserves of deposit money banks & 93 & 102 & 96 & 92 & 110 & 99 & 105 & 106 & 120 & 141 \\
\hline Other deposits & 21 & 25 & 24 & 63 & 25 & 100 & 30 & 7 & 19 & 47 \\
\hline \multicolumn{11}{|l|}{ Memorandum items: } \\
\hline & \multicolumn{10}{|c|}{ (Annual percentage change) } \\
\hline Reserve money & 26.0 & 10.2 & 10.5 & 13.4 & 6.2 & 14.3 & 6.4 & 13.2 & 7.8 & 12.5 \\
\hline Broad money & 25.9 & 26.4 & 28.1 & 17.4 & 23.1 & 18.3 & 18.8 & 16.9 & 12.9 & 12.5 \\
\hline Net credit to the government & 17.6 & 71.9 & 43.8 & 56.5 & 38.0 & 65.8 & 14.8 & 35.5 & 26.6 & 23.5 \\
\hline Claims on private sector & 39.4 & 56.8 & 61.7 & 11.7 & 56.7 & 14.3 & 44.3 & 15.5 & 31.8 & 18.0 \\
\hline Velocity (GDP/M2) & 4.4 & 4.1 & 4.4 & 4.5 & 4.2 & 4.2 & 4.1 & 4.0 & 4.0 & 4.0 \\
\hline Money multiplier (M2/base money) & 2.7 & 3.1 & 3.2 & 3.0 & 3.3 & 2.9 & 3.2 & 3.2 & 3.2 & 3.2 \\
\hline Gross Reserves (in US $\$$ millions) & 215.5 & 209.5 & 202.5 & 187.3 & 207.7 & 189.3 & 341.8 & 218.0 & 333.5 & 338.5 \\
\hline Excluding new SDR allocations & 215.5 & 209.5 & 202.5 & 187.3 & 207.7 & 189.3 & 213.7 & 218.0 & 205.4 & 210.4 \\
\hline
\end{tabular}

Sources: Sierra Leonean authorities; and IMF staff estimates and projections.

IMF Country Report No. 09/215.

${ }^{2}$ Gross foreign assets have been increased by US $\$ 128$ million, representing the new SDR allocations received by the Bank of Sierra Leone (BSL) in August and September 2009.

In line with actual BSL's recording practices, the counterpart of the new SDR allocations is BSL's capital liabilities, which are accounted for in other items net of the monetary survey. 
Table 5. Sierra Leone: Balance of Payments, 2007-13

(US\$ millions, unless otherwise indicated)

\begin{tabular}{|c|c|c|c|c|c|c|c|c|}
\hline & 2007 & 2008 & & 2009 & 2010 & 2011 & 2012 & 2013 \\
\hline & & Est. & Prog. ${ }^{1}$ & Rev. Prog. & & Proj. & & \\
\hline Current account balance & -57.2 & -207.7 & -178.3 & -182.3 & -172.2 & -162.7 & -168.8 & -170.8 \\
\hline $\begin{array}{l}\text { Balance on goods } \\
\text { Exports, f.o.b. } \\
\text { Of which: rutile } \\
\text { bauxite }\end{array}$ & $\begin{array}{r}-94.2 \\
282.5 \\
39.4 \\
32.7\end{array}$ & $\begin{array}{r}-213.2 \\
256.6 \\
39.0 \\
28.1\end{array}$ & $\begin{array}{r}-199.9 \\
180.7 \\
28.7 \\
17.9\end{array}$ & $\begin{array}{r}-206.4 \\
246.7 \\
32.4 \\
14.7\end{array}$ & $\begin{array}{r}-211.6 \\
294.6 \\
31.9 \\
16.2\end{array}$ & $\begin{array}{r}-210.1 \\
345.4 \\
35.9 \\
18.9\end{array}$ & $\begin{array}{r}-220.1 \\
380.7 \\
37.1 \\
19.5\end{array}$ & $\begin{array}{r}-225.0 \\
414.3 \\
40.9 \\
21.3\end{array}$ \\
\hline $\begin{array}{l}\text { diamonds }^{2} \\
\text { coffee and cocoa }\end{array}$ & $\begin{array}{r}168.6 \\
13.3\end{array}$ & $\begin{array}{r}117.6 \\
16.5\end{array}$ & $\begin{array}{r}70.5 \\
7.1\end{array}$ & $\begin{array}{l}96.3 \\
44.0\end{array}$ & $\begin{array}{r}140.8 \\
58.5\end{array}$ & $\begin{array}{r}178.3 \\
62.6\end{array}$ & $\begin{array}{r}206.4 \\
66.4\end{array}$ & $\begin{array}{r}223.0 \\
74.8\end{array}$ \\
\hline Imports, f.o.b. & -376.6 & -469.7 & -380.6 & -453.1 & -506.2 & -555.6 & -600.8 & -639.3 \\
\hline Of which: petroleum & -105.7 & -151.5 & -92.4 & -100.1 & -117.3 & -129.8 & -137.6 & -147.3 \\
\hline rice & -20.9 & -52.1 & -48.0 & -47.0 & -46.1 & -45.1 & -44.2 & -43.3 \\
\hline $\begin{array}{l}\text { Balance on services } \\
\text { Credit } \\
\text { Debit }\end{array}$ & $\begin{array}{r}-22.6 \\
63.1 \\
-85.7\end{array}$ & $\begin{array}{r}-41.9 \\
62.8 \\
-104.7\end{array}$ & $\begin{array}{r}-42.1 \\
57.8 \\
-99.9\end{array}$ & $\begin{array}{r}-43.1 \\
57.8 \\
-100.9\end{array}$ & $\begin{array}{r}-47.2 \\
61.3 \\
-108.5\end{array}$ & $\begin{array}{r}-50.4 \\
65.0 \\
-115.4\end{array}$ & $\begin{array}{r}-54.4 \\
68.9 \\
-123.4\end{array}$ & $\begin{array}{r}-59.0 \\
73.0 \\
-132.0\end{array}$ \\
\hline $\begin{array}{l}\text { Income } \\
\text { Credit } \\
\text { Debit }\end{array}$ & $\begin{array}{r}-34.6 \\
7.8 \\
-42.5\end{array}$ & $\begin{array}{r}-39.2 \\
8.2 \\
-47.3\end{array}$ & $\begin{array}{r}-40.0 \\
8.5 \\
-48.5\end{array}$ & $\begin{array}{r}-40.7 \\
8.5 \\
-49.3\end{array}$ & $\begin{array}{r}-41.7 \\
8.9 \\
-50.6\end{array}$ & $\begin{array}{r}-41.7 \\
9.3 \\
-51.0\end{array}$ & $\begin{array}{r}-42.2 \\
9.7 \\
-51.9\end{array}$ & $\begin{array}{r}-42.9 \\
10.2 \\
-53.1\end{array}$ \\
\hline Current transfers & 94.2 & 86.5 & 103.7 & 107.9 & 128.3 & 139.5 & 147.9 & 156.2 \\
\hline Public (net) & 58.6 & 48.2 & 78.7 & 73.2 & 73.4 & 79.9 & 83.2 & 85.4 \\
\hline Of which: HIPC grants & 12.0 & 4.5 & 3.5 & 3.8 & 3.6 & 4.0 & 3.6 & 2.0 \\
\hline Private (net) ${ }^{4}$ & 35.6 & 38.3 & 24.9 & 34.7 & 54.9 & 59.6 & 64.7 & 70.8 \\
\hline Current account balance, excl. public transfers & -115.8 & -255.9 & -257.0 & -255.5 & -245.6 & -242.6 & -252.0 & -256.1 \\
\hline Capital and financial account & 88.5 & 183.6 & 149.8 & 269.1 & 168.5 & 172.7 & 186.0 & 189.9 \\
\hline Capital account & 472.1 & 42.7 & 75.0 & 71.3 & 67.3 & 75.2 & 81.7 & 87.5 \\
\hline Project grants & 23.3 & 40.5 & 71.4 & 68.9 & 64.7 & 72.3 & 78.5 & 84.0 \\
\hline Debt forgiveness (MDRI relief from IDA and AfDB) & 445.9 & 0.0 & 0.0 & 0.0 & 0.0 & 0.0 & 0.0 & 0.0 \\
\hline Private capital transfers & 3.0 & 2.1 & 3.6 & 2.4 & 2.6 & 2.9 & 3.1 & 3.5 \\
\hline Financial account & -383.6 & 141.0 & 74.8 & 197.8 & 101.2 & 97.6 & 104.3 & 102.5 \\
\hline Direct investment and portfolio investment & 69.7 & 53.0 & 22.1 & 33.4 & 35.8 & 38.4 & 39.8 & 37.3 \\
\hline $\begin{array}{l}\text { Other investment } \\
\text { Public sector (net) } \\
\text { Of which: disbursements }\end{array}$ & $\begin{array}{r}-453.4 \\
-447.1 \\
20.3\end{array}$ & $\begin{array}{l}87.9 \\
48.1 \\
54.7\end{array}$ & $\begin{array}{l}52.7 \\
49.1 \\
57.5\end{array}$ & $\begin{array}{r}164.4 \\
53.8 \\
64.5\end{array}$ & $\begin{array}{l}65.4 \\
51.4 \\
60.7\end{array}$ & $\begin{array}{l}59.1 \\
69.8 \\
82.3\end{array}$ & $\begin{array}{l}64.5 \\
80.2 \\
88.5\end{array}$ & $\begin{array}{l}65.2 \\
76.1 \\
84.0\end{array}$ \\
\hline project loans & 20.3 & 54.7 & 57.5 & 54.5 & 50.7 & 72.3 & 78.5 & 84.0 \\
\hline amortization due & -467.5 & -6.7 & -8.4 & -10.7 & -9.3 & -12.5 & -8.3 & -7.9 \\
\hline Monetary authorities & 0.0 & 0.0 & 0.0 & 128.1 & 0.0 & 0.0 & 0.0 & 0.0 \\
\hline Private sector (net) & 0.0 & 0.0 & 0.0 & 0.0 & 0.0 & 0.0 & 0.0 & 0.0 \\
\hline Change in net foreign assets of commercial banks & -45.8 & -2.2 & 3.9 & -19.8 & 18.0 & -10.2 & -12.2 & 2.0 \\
\hline Other, including errors and omissions & 39.6 & 42.1 & -0.3 & 2.3 & -4.0 & -0.5 & -3.5 & -13.0 \\
\hline Overall balance & 31.3 & -24.0 & -28.5 & 86.7 & -3.7 & 10.1 & 17.2 & 19.2 \\
\hline Change in net foreign reserve assets of the central bank $(-=$ inc & -31.3 & 24.0 & 12.7 & -86.7 & 3.7 & -10.1 & -17.2 & -19.2 \\
\hline Change in reserve assets $(-=$ increase $)$ & -31.3 & $\begin{array}{r}6.0 \\
18.0\end{array}$ & -8.6 & $\begin{array}{r}-124.1 \\
373\end{array}$ & $\begin{array}{r}-5.0 \\
8.7\end{array}$ & $\begin{array}{l}-5.0 \\
-51\end{array}$ & $\begin{array}{r}-10.0 \\
-72\end{array}$ & $\begin{array}{r}-12.0 \\
-72\end{array}$ \\
\hline $\begin{array}{l}\text { Net Fund credit } \\
\text { Fund PRGF disbursement }\end{array}$ & $\begin{array}{l}0.0 \\
0.0\end{array}$ & $\begin{array}{l}18.0 \\
18.0\end{array}$ & $\begin{array}{l}21.3 \\
21.3\end{array}$ & $\begin{array}{l}37.3 \\
37.3\end{array}$ & $\begin{array}{r}8.7 \\
10.9\end{array}$ & $\begin{array}{r}-5.1 \\
0.0\end{array}$ & $\begin{array}{r}-7.2 \\
0.0\end{array}$ & $\begin{array}{r}-7.2 \\
0.0\end{array}$ \\
\hline Repayments & 0.0 & 0.0 & 0.0 & 0.0 & -2.2 & -5.1 & -7.2 & -7.2 \\
\hline Financing gap & 0.0 & 0.0 & 15.8 & 0.0 & 0.0 & 0.0 & 0.0 & 0.0 \\
\hline Augmentation of PRGF access & 0.0 & 0.0 & 15.8 & 0.0 & 0.0 & 0.0 & 0.0 & 0.0 \\
\hline Remaining gap & 0.0 & 0.0 & 0.0 & 0.0 & 0.0 & 0.0 & 0.0 & 0.0 \\
\hline Memorandum items: & & & rcent of G & SDP unless ot & se indicat & & & \\
\hline Current account & -3.4 & -10.6 & -8.5 & -9.4 & -8.7 & -7.5 & -7.2 & -6.8 \\
\hline Current account, excluding public transfers & -7.0 & -13.1 & -12.3 & -13.2 & -12.4 & -11.2 & -10.7 & -10.2 \\
\hline Overall balance of payments & 1.9 & -1.2 & -1.4 & 4.5 & -0.2 & 0.5 & 0.7 & 0.8 \\
\hline MDRI debt service savings (US\$ millions) & 21.3 & 29.2 & 33.1 & 33.1 & 39.1 & 39.4 & 26.1 & 18.0 \\
\hline Of which: IMF & 16.2 & 24.1 & 28.0 & 28.0 & 33.9 & 33.7 & 18.9 & 10.1 \\
\hline AfDF & 1.8 & 1.9 & 1.9 & 1.9 & 1.9 & 2.2 & 2.5 & 2.7 \\
\hline IDA & 3.2 & 3.2 & 3.2 & 3.2 & 3.3 & 3.5 & 4.7 & 5.2 \\
\hline Gross official reserves (US\$ millions) & 215.5 & 209.5 & 218.0 & 333.5 & 338.5 & 343.5 & 353.5 & 365.5 \\
\hline Gross official reserves (months of imports) ${ }^{5}$ & 4.5 & 4.5 & 5.0 & 6.5 & 6.1 & 5.7 & 5.5 & 5.4 \\
\hline GDP (US\$ millions) & 1663.7 & 1954.3 & 2091.1 & 1939.2 & 1982.8 & 2158.7 & 2344.2 & 2507.6 \\
\hline
\end{tabular}

Sources: Sierra Leonean authorities; and IMF staff estimates and projections.

1 IMF Country Report No. 09/215.

${ }^{2}$ Includes unrecorded diamond exports estimated from partner-country data.

${ }^{3}$ Official interest payments due, including Fund charges.

${ }^{4}$ Includes worker remittances and transfers to NGOs.

${ }^{5}$ Months of imports of total goods and services of subsequent year. 
Table 6. Sierra Leone: Indicators of Capacity to Repay the Fund, 2007-16

(US\$ millions, unless otherwise indicated)

\begin{tabular}{|c|c|c|c|c|c|c|c|c|c|c|}
\hline & 2007 & 2008 & 2009 & 2010 & 2011 & 2012 & 2013 & 2014 & 2015 & 2016 \\
\hline & & & \multicolumn{8}{|c|}{ Proj. } \\
\hline \multicolumn{11}{|l|}{ Fund credit outstanding (end of period) } \\
\hline SDR millions & 23.1 & 34.5 & 58.9 & 64.5 & 61.2 & 56.6 & 52.0 & 43.9 & 32.8 & 22.9 \\
\hline US\$ millions & 35.4 & 54.6 & 90.2 & 100.4 & 95.1 & 87.9 & 80.6 & 67.9 & 50.4 & 34.9 \\
\hline Percent of quota & 22.3 & 33.3 & 56.8 & 62.2 & 59.0 & 54.6 & 50.1 & 42.3 & 31.6 & 22.0 \\
\hline Fund obligations based on existing and prospective credit & 0.2 & 0.2 & 0.2 & 2.7 & 5.6 & 7.6 & 7.6 & 13.0 & 17.4 & 15.4 \\
\hline Charges and interests & 0.2 & 0.2 & 0.2 & 0.5 & 0.5 & 0.5 & 0.4 & 0.4 & 0.3 & 0.2 \\
\hline Principal & 0.0 & 0.0 & 0.0 & 2.2 & 5.1 & 7.2 & 7.2 & 12.6 & 17.0 & 15.1 \\
\hline \multicolumn{11}{|l|}{ Fund credit outstanding as percent of: } \\
\hline Exports of goods and services & 10.2 & 17.1 & 29.6 & 28.2 & 23.2 & 19.5 & 16.5 & 13.0 & 8.8 & 5.4 \\
\hline Total external debt & 6.7 & 9.0 & 12.9 & 13.2 & 16.1 & 13.2 & 11.0 & 8.6 & 6.0 & 3.9 \\
\hline Gross official reserves & 16.4 & 26.0 & 26.6 & 28.4 & 26.0 & 23.7 & 21.5 & 17.8 & 13.1 & 8.9 \\
\hline \multicolumn{11}{|l|}{ Fund obligations as percent of: } \\
\hline Exports of goods and services & 0.1 & 0.1 & 0.1 & 0.8 & 1.4 & 1.7 & 1.6 & 2.5 & 3.0 & 2.4 \\
\hline Gross international reserves & 0.1 & 0.1 & 0.1 & 0.8 & 1.5 & 2.1 & 2.0 & 3.4 & 4.5 & 3.9 \\
\hline \multicolumn{11}{|l|}{ Memorandum items: } \\
\hline Exports of goods and services (US\$ millions) & 345.6 & 319.4 & 304.5 & 355.9 & 410.5 & 449.6 & 487.3 & 521.6 & 574.0 & 641.2 \\
\hline Gross international reserves (in months of imports) & 4.5 & 4.5 & 6.1 & 5.5 & 5.4 & 5.1 & 4.8 & 4.5 & 4.2 & 3.9 \\
\hline Debt service due after debt relief, incl. to IMF (in percent of exports) & 4.3 & 1.9 & 3.3 & 3.7 & 4.5 & 3.8 & 3.9 & 4.2 & 5.3 & 5.0 \\
\hline GDP (US\$ millions) & $1,663.7$ & $1,954.3$ & $1,939.2$ & $1,982.8$ & $2,158.7$ & $2,344.2$ & $2,507.6$ & $2,654.8$ & $2,816.2$ & $2,990.3$ \\
\hline
\end{tabular}

Sources: Sierra Leonean authorities; and IMF staff estimates and projections. 
Table 7. Sierra Leone: Schedule of Disbursements Under the PRGF Arrangement, 2006-10 (SDR millions)

\begin{tabular}{|c|c|c|}
\hline Date & Disbursements & Subject to \\
\hline May 2006 & 4.71 & Approval of arrangement \\
\hline December 2006 & 4.40 & $\begin{array}{l}\text { First review and performance criteria (PCs) for } \\
\text { end-June } 2006\end{array}$ \\
\hline July 2008 & 4.40 & $\begin{array}{l}\text { Completion of the second review and waivers } \\
\text { for the nonobservance of end-December } 2006 \\
\text { PCs }\end{array}$ \\
\hline December 2008 & 7.00 & $\begin{array}{l}\text { Third review and quantitative PCs for end- } \\
\text { June 2008, and structural PC for end- } \\
\text { September 2008, as described in the } \\
\text { June 5, } 2008 \text { MEFP, Tables } 1 \text { and } 2\end{array}$ \\
\hline June 2009 & 12.185 & $\begin{array}{l}\text { Fourth review and quantitative PCs for end- } \\
\text { December } 2008 \text { and structural PC as described } \\
\text { in the June 5, } 2008 \text { MEFP, Table } 2\end{array}$ \\
\hline December 2009 & 12.185 & $\begin{array}{l}\text { Fifth review and quantitative PCs for end- } \\
\text { June } 2009\end{array}$ \\
\hline May 2010 & 7.00 & $\begin{array}{l}\text { Sixth review and quantitative PCs for end- } \\
\text { December } 2009\end{array}$ \\
\hline Total disbursements & 51.88 & \\
\hline
\end{tabular}


Table 8. Sierra Leone: Millennium Development Goals

\begin{tabular}{|c|c|c|c|c|c|}
\hline & 1990 & 1995 & 2000 & 2005 & 2008 \\
\hline \multicolumn{6}{|l|}{ Goal 1: Eradicate extreme poverty and hunger } \\
\hline Employment to population ratio, $15+$, total $(\%)$ & 64 & 65 & 65 & 64 & 64 \\
\hline Employment to population ratio, ages $15-24$, total (\%) & 39 & 41 & 44 & 41 & 42 \\
\hline GDP per person employed (annual \% growth) & -20 & -9 & 1 & 4 & 4 \\
\hline Income share held by lowest $20 \%$ & 1.1 & .. & .. & 6.1 & .. \\
\hline Malnutrition prevalence, weight for age (\% of children under 5 ) & .. & .. & 24.7 & 28.3 & .. \\
\hline Poverty gap at $\$ 1.25$ a day (PPP) (\%) & 45 & .. & .. & 20 & .. \\
\hline Poverty headcount ratio at $\$ 1.25$ a day (PPP) ( $\%$ of population) & 63 & .. & .. & 53 & .. \\
\hline Prevalence of undernourishment (\% of population) & 45 & 43 & .. & 47 & .. \\
\hline Vulnerable employment, total (\% of total employment) & .. & .. & .. & .. & .. \\
\hline \multicolumn{6}{|l|}{ Goal 2: Achieve universal primary education } \\
\hline Literacy rate, youth female (\% of females ages $15-24$ ) & .. & .. &.. & 37 & 44 \\
\hline Literacy rate, youth male (\% of males ages $15-24)$ & .. & .. &.. & 60 & 64 \\
\hline Persistence to last grade of primary, total (\% of cohort) & .. & .. & .. & .. & .. \\
\hline Primary completion rate, total (\% of relevant age group) & .. & .. & .. & 81 & 81 \\
\hline Total enrollment, primary (\% net) & .. & .. & .. & .. & .. \\
\hline \multicolumn{6}{|l|}{ Goal 3: Promote gender equality and empower women } \\
\hline Proportion of seats held by women in national parliaments (\%) & .. & 6 & 9 & 15 & 13 \\
\hline Ratio of female to male enrollments in tertiary education & .. & .. & 40 & .. & .. \\
\hline Ratio of female to male primary enrollment & 69 & .. & 71 & 90 & 90 \\
\hline Ratio of female to male secondary enrollment & 57 & .. & 71 & 69 & 69 \\
\hline Share of women employed in the nonagricultural sector ( $\%$ of total nonagricultural employment) & .. & .. & .. & 23.2 & .. \\
\hline \multicolumn{6}{|l|}{ Goal 4: Reduce child mortality } \\
\hline Immunization, measles ( $\%$ of children ages $12-23$ months) & .. & .. & 37 & 67 & 67 \\
\hline Mortality rate, infant (per 1,000 live births) & 169 & 166 & 161 & 157 & 155 \\
\hline Mortality rate, under- 5 (per 1,000$)$ & 290 & 283 & 274 & 265 & 262 \\
\hline \multicolumn{6}{|l|}{ Goal 5: Improve maternal health } \\
\hline Adolescent fertility rate (births per 1,000 women ages $15-19$ ) & .. & 146 & 139 & 130 & 126 \\
\hline Births attended by skilled health staff ( $\%$ of total) & .. & .. & 42 & 43 & .. \\
\hline Contraceptive prevalence (\% of women ages $15-49$ ) & .. & .. & 4 & 5 & .. \\
\hline Maternal mortality ratio (modeled estimate, per 100,000 live births) & .. & .. & .. & 2,100 & .. \\
\hline Pregnant women receiving prenatal care (\%) & .. & .. & 68 & 81 & .. \\
\hline Unmet need for contraception (\% of married women ages $15-49$ ) & .. & .. &.. & .. & .. \\
\hline \multicolumn{6}{|l|}{ Goal 6: Combat HIVIAIDS, malaria, and other diseases } \\
\hline Children with fever receiving antimalarial drugs ( $\%$ of children under age 5 with fever) & .. & .. & 61 & 52 & .. \\
\hline Condom use, population ages $15-24$, female ( $\%$ of females ages $15-24)$ & .. & .. & .. & .. & .. \\
\hline Condom use, population ages $15-24$, male (\% of males ages $15-24)$ & .. & .. &.. & .. & .. \\
\hline Incidence of tuberculosis (per 100,000 people) & 207 & 279 & 377 & 509 & 574 \\
\hline Prevalence of HIV, female (\% ages $15-24)$ & .. & .. & .. & 1.3 & 1.3 \\
\hline Prevalence of HIV, male (\% ages $15-24)$ & .. & .. & .. & 0 & 0 \\
\hline Prevalence of HIV, total (\% of population ages 15-49) & 0.2 & 1.0 & 1.2 & 1.6 & 1.7 \\
\hline Tuberculosis cases detected under DOTS (\%) & .. & 29 & 33 & 36 & 37 \\
\hline \multicolumn{6}{|l|}{ Goal 7: Ensure environmental sustainability } \\
\hline $\mathrm{CO} 2$ emissions ( $\mathrm{kg}$ per PPP \$ of GDP) & 0.2 & 0.3 & 0.4 & 0.3 & .. \\
\hline CO2 emissions (metric tons per capita) & 0.1 & 0.1 & 0.1 & 0.2 & .. \\
\hline Forest area (\% of land area) & 43 & 41 & 40 & 38 & .. \\
\hline Improved sanitation facilities (\% of population with access) & .. & 12 & 12 & 11 & 11 \\
\hline Improved water source (\% of population with access) & .. & 57 & 57 & 53 & 53 \\
\hline Marine protected areas, ( $\%$ of surface area) & .. & .. & .. & .. & .. \\
\hline Nationally protected areas ( $\%$ of total land area) & .. & .. & .. & 4.1 & 4.1 \\
\hline \multicolumn{6}{|l|}{ Goal 8: Develop a global partnership for development } \\
\hline Aid per capita (current US\$) & 15 & 51 & 43 & 69 & 99 \\
\hline Debt service (PPG and IMF only, \% of exports, excluding workers' remittances) & 10 & 62 & 46 & 9 & 4 \\
\hline Internet users (per 100 people) & 0.0 & 0.0 & 0.1 & 0.2 & 0.3 \\
\hline Mobile cellular subscriptions (per 100 people) & 0 & 0 & 0 & 14 & 18 \\
\hline Telephone lines (per 100 people) & 0 & 0 & 0 & .. & .. \\
\hline \multicolumn{6}{|l|}{ Other } \\
\hline Fertility rate, total (births per woman) & 5.5 & 5.5 & 5.4 & 5.3 & 5.2 \\
\hline GNI per capita, Atlas method (current US\$) & 190 & 200 & 150 & 230 & 320 \\
\hline GNI, Atlas method (current US\$) (billions) & 0.8 & 0.8 & 0.6 & 1.2 & 1.8 \\
\hline Gross capital formation (\% of GDP) & 10.0 & 5.6 & 6.9 & 17.4 & 19.7 \\
\hline Life expectancy at birth, total (years) & 40 & 39 & 43 & 46 & 48 \\
\hline Literacy rate, adult total (\% of people ages 15 and above) & .. & .. & .. & 35 & 38 \\
\hline Population, total (millions) & 4.1 & 4.0 & 4.2 & 5.1 & 5.6 \\
\hline
\end{tabular}

Source: World Development Indicators database, 2009 
Table 9. Sierra Leone: Financial Soundness Indicators of the Banking System, 2005-09

\begin{tabular}{|c|c|c|c|c|c|}
\hline & 2005 & 2006 & 2007 & 2008 & $\begin{array}{r}2009 \\
\text { June } \\
\end{array}$ \\
\hline & \multicolumn{5}{|c|}{ (Percent, end of period, unless otherwise indicated) } \\
\hline \multicolumn{6}{|l|}{ Capital adequacy } \\
\hline Regulatory capital ratio $^{1}$ & 35.7 & 33.3 & 35.0 & 43.5 & 36.2 \\
\hline Regulatory tier 1 capital ratio ${ }^{2}$ & 10.3 & 17.0 & 16.7 & 18.7 & 17.5 \\
\hline \multicolumn{6}{|l|}{ Asset quality } \\
\hline Nonperforming loans to total gross loans & 26.8 & 27.8 & 31.7 & 23.3 & 17.8 \\
\hline Nonperforming loans (net of provisions) to regulatory capital & 26.8 & 24.1 & 37.8 & 25.4 & 24.4 \\
\hline Loan loss provisions to NPLs (net of accrued interest) & 10.3 & 59.7 & 44.5 & 54.4 & 35.8 \\
\hline \multicolumn{6}{|l|}{ Earnings and profitability } \\
\hline Return on assets & 8.1 & 5.8 & 3.1 & 2.2 & 1.0 \\
\hline Return on equity & 28.0 & 17.0 & 10.3 & 7.2 & 2.9 \\
\hline Interest spread $^{3}$ & 13.4 & 13.4 & 15.2 & --- & --- \\
\hline \multicolumn{6}{|l|}{ Liquidity } \\
\hline Ratio of net loans to total deposits & 27.5 & 26.5 & 27.7 & 33.9 & 40.0 \\
\hline Liquidity ratio $^{4}$ & 53.0 & 58.9 & 53.3 & 62.0 & 57.2 \\
\hline Statutory minimum liquidity ratio ${ }^{4} 5$ & 34.0 & 28.5 & 25.7 & 25.0 & 23.3 \\
\hline Share of foreign exchange deposits in total deposits & 30.7 & 32.4 & 36.5 & 32.2 & 30.8 \\
\hline & \multicolumn{5}{|c|}{ (Number of banks not complying) } \\
\hline \multicolumn{6}{|l|}{ Prudential ratios at year-end } \\
\hline Capital adequacy & 0 & 0 & 0 & 0 & 0 \\
\hline Minimum liquidity ratio & 0 & 0 & 0 & 0 & 1 \\
\hline Minimum capital & 0 & 1 & 1 & 1 & 0 \\
\hline Limit of single large exposure ${ }^{6}$ & 0 & 2 & 1 & 2 & 3 \\
\hline \multicolumn{6}{|l|}{ Memorandum Item: } \\
\hline Number of banks & 7 & 8 & 10 & 13 & 13 \\
\hline
\end{tabular}

Source: Bank of Sierra Leone.

${ }^{1}$ Capital requirement over risk-weighted assets (solvency ratio).

${ }^{2}$ Core capital (Tier I) over total assets.

${ }^{3}$ Average lending rate minus average saving deposit rate.

${ }^{4}$ Calculated taking into account both domestic currency and foreign currency deposits. Liquid assets include domestic currency cash in vault, claims on the BSL, claims on discount houses, and government securities.

${ }^{5}$ Effective November 2007, minimum liquidity includes 40 percent of demand deposits and 20 percent of quasi money to be held in either cash or treasury bills.

${ }^{6}$ A single large exposure of an institution is any exposure that is 2 percent or more of its capital base. 


\section{APPENDIX I-LETTER OF INTENT: SIERRA LEONE}

December 7, 2009

Mr. Dominique Strauss-Kahn

Managing Director

International Monetary Fund

Washington, D.C. 20431

U.S.A.

Dear Mr. Strauss-Kahn:

The attached Memorandum of Economic and Financial Policies (MEFP) supplements the one attached to my letter to you dated June 4, 2009. It describes recent economic developments and progress in the implementation of the PRGF-supported program during the first half of 2009 and presents policies planned for the remainder of 2009 and 2010.

Program implementation during the first half of this year was satisfactory. All end-June 2009 quantitative performance criteria were met. The structural benchmark for adoption by the Cabinet of the implementation decree for the new customs law and regulations by end-June was also met, although with delays caused by further technical clarifications required by the Cabinet on the new law and regulations.

The standard continuous performance criterion on nonintroduction and modification of multiple currency practices (MCPs) was not observed due to the introduction of a special rice window in August 2009 in the weekly foreign exchange auction conducted by the Bank of Sierra Leone (BSL); this followed the introduction of a special oil window in the foreign exchange auction in November 2008. Having been informed by staff that these windows resulted in MCPs, the BSL moved speedily to unify the auction into a single competitive window by end-September 2009. On the basis of this corrective action, the Government requests a waiver for the nonobservance of the continuous performance criteria on nonintroduction and modification of MCPs for the August 2009 introduction of the special rice window.

In light of the above, the Government of Sierra Leone (GoSL) requests that the sixth disbursement be made available upon completion of the fifth review under the PRGF arrangement.

Since the fourth review of the PRGF arrangement was approved by the IMF Executive Board in June, we have been faced with unanticipated spending pressures, including costs related to the completion of the Bumbuna hydropower project, which has prompted an extension of the Emergency Power Project by another three months, and the deployment of our military force to the Dafour UN military force. Part of the new spending needs will be covered by expected 
additional external budgetary assistance, but additional domestic financing will also be necessary. The global economic downturn has significantly reduced foreign exchange inflows into Sierra Leone and the leone has depreciated by 20 percent against the US dollar since the beginning of the year. The BSL has intervened moderately in the foreign exchange market. For these reasons, we request that the targets for end-December 2009 on net domestic credit to government, net domestic assets, domestic primary fiscal balance, and gross foreign exchange reserves be modified.

The GoSL believes that the policies set forth in the attached MEFP and Technical Memorandum of Understanding (TMU) are adequate to achieve the objectives of its program for 2009, but stands ready to take any further measures that become necessary for this purpose, in consultation with the Fund. The GoSL will also continue to provide the staff of the IMF the information required to accurately assess Sierra Leone's progress in executing the policies contained in the attached MEFP. The sixth and final review shall take place in April 2010; at that time we anticipate that preliminary discussions on a successor Fundsupported program could take place. Furthermore, the GoSL will continue to consult with the IMF on its economic and financial policies, in accordance with the IMF's policies on such consultations.

The GoSL agrees, in line with its commitment to transparency and accountability, to the publication of this letter, its attachments, and the related staff report in accordance with the procedures for publication.

Very truly yours,

Samura M. W. Kamara
Minister of Finance and Economic Development

Attachments 


\section{Attachment I. Sierra Leone: Memorandum of EConomic and Financial Policies}

\section{INTRODUCTION}

1. This memorandum updates the policies presented in our Memorandum of Economic and Financial Policies (MEFP) of June 4, 2009 (IMF Country Report $N^{\circ}$ 09/215). It reviews recent performance under the program through June 2009 and outlines the macroeconomic policies and structural reforms that the Government of Sierra Leone (GoSL) will pursue during the remainder of 2009 and in 2010.

\section{RECENT ECONOMIC DEVELOPMENTS}

2. The economic slowdown continued in 2009. Since the last quarter of 2008, the Sierra Leonean economy has been negatively affected by slowing global demand, falling export prices, particularly for diamond and bauxite, and declining inward remittances and other foreign inflows. The slowdown in economic activity reduced real GDP growth to 5.5 percent in 2008, compared with a projection of 6.4 percent. While economic activity continued to weaken during the first half of 2009, particularly in the mining sector, in recent months there have been signs of a pick up in exports of diamonds and agriculture products. Real GDP growth is expected to reach 4 percent in 2009, helped by higher agricultural productivity and increased public investment in basic infrastructure. Lower fuel prices and buoyant domestic food production have eased inflation, which declined from 12.2 percent in December 2008 to 8.3 percent in August 2009. However, upside risks to inflation remain, following the depreciation of the leone against the US dollar by about 20 percent since the beginning of this year.

3. Budget execution during the first half of 2009 was prudent. Domestic revenue collections exceeded the target and, despite delays in external budgetary support, prudent cash-budget controls helped to contain the fiscal deficit and domestic financing. The domestic primary deficit was 1.3 percent of GDP — about 0.5 percent of GDP below the program target for the first half of the year.

\section{Monetary policy remained tight to contain inflation and maintain financial} stability. However, the conduct of monetary policy has been challenged by the depreciation of the leone and delays in donor disbursements. Reserve money growth remained below the program target, helped by the issuance of Le 20 billions of treasury bills, which were converted from BSL's holding of noninterest-bearing government securities.

5. The BSL has strengthened its capacity to improve its liquidity management. The conversion of noninterest-bearing government securities into marketable securities and an increase in the amount of foreign exchange auction helped to mop up excess liquidity. In order to help develop interbank market, the BSL phased out the discount window with the commencement of repos and reverse repos operations in June 2009. 
6. Bank credit to the private sector continued to grow strongly. The increase in competition among banks is the main reason for this growth. The entry of three new banks in 2008 also contributed to maintain a high demand for already oversubscribed government securities, which helped to maintain low interest rates. The average effective yield on Treasury bills fell from 21.3 percent at end-2007 to about 9.1 at end-2008, but since then it has risen again to about 10.8 percent by end-September 2009 .

7. The quality of the loan portfolio remains a concern. Although nonperforming loans (NPLs) relative to gross loans halved between March 2008 and June 2009, they remain high at 17.8 percent. Using a less restrictive measure, which is more in line with international standards, the ratio of NPLs relative to gross loans still stands at 10.5 percent.

8. After remaining fairly stable over the past three years, the leone depreciated by 20 percent against the U.S dollar during the first seven months of 2009 . This reflected a drop in the availability of foreign exchange as export receipts and remittances continued to decline. In response to an accelerated depreciation in July and August, the BSL intervened moderately in the foreign exchange market. Gross international reserves amounted to about US\$208 million (about 5 months of imports) at end-June 2009. Sierra Leone's share of the recent SDR allocations, equivalent to about US\$128 million, was allocated to the BSL in late August and early September, bringing gross international reserves to US\$336 million at endSeptember.

9. Program implementation in the first half of $\mathbf{2 0 0 9}$ was satisfactory. All end-June quantitative performance criteria were met. On the structural targets, the continuous structural benchmark on the monthly meeting of the Monetary Policy Committee was met. Although, the structural benchmark for adoption by the Cabinet of the implementation decree for the new customs law and regulations was not observed as envisaged in June, the measure was implemented in early September 2009. Of the two structural benchmarks for September 2009 , only one has been met. The benchmark on the BSL adoption of new off-site surveillance guidelines was not observed because revisions of the Banking Act and Other Financial Services Act have not yet been completed (Table 2).

\section{MACROECONOMiC FrameWork}

10. The macroeconomic outlook for 2009-10 has been revised to take into account the impact of recent domestic and external developments. Projected real GDP growth remains at 4 percent in 2009, while annual inflation is projected to rise slightly to 9.5 percent at year-end, compared with the 9 percent previously envisaged. Inflation is targeted to decline to 8 percent in 2010. The recent rebound in mining production and higher exports of agriculture products (in particular cocoa and coffee) combined with the anticipated slow growth in imports would contribute to reducing the external current account deficit from 10.7 percent in 2008 to about 9.4 percent in 2009 . Gross official reserves, including the 
recent SDR allocation, are projected to stay at about 6.5 months of import coverage by end2009.

\section{The policies for 2009-10 will be anchored by the Agenda for Change, as} presented in PRSP-II completed in June this year. The four key priority areas identified in the PRSP are: (i) provision of a reliable power supply in the country; (ii) support for agriculture, agri-business, and fisheries with an emphasis on increasing the productivity of the poor; (iii) rehabilitation of the transportation network to facilitate the movement of goods and people and thereby promote economic activity; and (iv) improved coverage of basic social services through decentralization and more support for the social protection needs of the poor and vulnerable. The program is aimed at enhancing fiscal efforts to mobilize revenues, which would help expanding investment spending to support the implementation of the PRS. In addition, it supports the efforts to (i) promoting efficiency, transparency and accountability in the use of public resources through enhanced public financial management and governance; and (ii) improving the investment climate by ensuring the provision of electricity in a fiscally sustainable manner.

\section{Policies FOR THe ReMAinder OF 2009}

\section{While domestic revenue remains on track, unanticipated expenditure needs in} priority areas will raise the fiscal deficit above the target for 2009. Domestic revenue is projected to increase slightly to 11.3 percent of GDP. This reflects higher import duties on account of an increase in the tax base but lower fuel excise collections. Also, as programmed, steps are underway to ensure an effective transfer of all off-budget revenue collected by Ministries, Departments, and Agencies (MDAs) to the Consolidated Revenue Fund, in line with the Finance Act passed by the Parliament in August 2009. On the expenditure side, delays in the completion of the Bumbuna hydroelectric project has necessitated an extension of the Emergency Power Project by three months for a total cost of about US\$5 million ( 0.3 percent of GDP). In addition, unanticipated spending amounting to about 0.3 percent of GDP will be required to cover our contribution to the participation of Sierra Leonean military forces to the UN mission in Darfur, additional transfers to universities, payments of outstanding commitments related to the Bumbuna project, and counterpart funds to key road projects. The payment by the National Power Authority (NPA) of US\$4 million to an external power company, with whom a recent settlement on an outstanding claim was reached, is expected to lower NPA's contribution to cover past fuel purchases for emergency power generation (about 0.2 percent of GDP). The resulting financing gap from these unanticipated costs is 0.8 percent of GDP. The gap will be closed by additional budgetary assistance from the European Commission ( 0.3 percent of GDP) and additional bank financing.

\section{The government will step up efforts to improve tax administration and broaden}

the tax base. Measures include (i) establishing the domestic tax department (structural benchmark for end-December 2009); (ii) integrating the Good and Service Tax (GST) 
administration within the Large Taxpayer Office (structural benchmark for end-December 2009), (iii) enforcing the provisions in the existing tax legislation with the aim of eliminating tax evasion and ad-hoc tax exemptions; (iv) intensifying field audits and enforcement of the payment of tax arrears (with interest and penalties on under/late payment of tax); and (v) eliminating discretionary duty waivers and tax exemptions.

\section{Monetary policy will remain tight to contain inflation expectations and pressure} on the exchange rate. The aim is to maintain single-digit inflation in 2009. To this end, reserve money is targeted to grow at about 8 percent. The BSL will actively conduct open market operations to control the growth of reserve money - and this may require an increase in the interest rates on treasury bills and repos. Given the current foreign exchange shortages, the original targeted increase in foreign exchange reserves during the second half of 2009 is no longer attainable. Gross foreign reserves, excluding the new SDR allocation, are now targeted at US\$205 million by end-December 2009 (about US\$13 million below the original target). Including the SDR allocation, gross foreign reserves will be targeted at US $\$ 333$ million. The Monetary Policy Committee (MPC) will continue to meet on a monthly basis and coordination between the MoFED and the BSL will be strengthened.

15. Exchange rate flexibility will be maintained. BSL will limit its interventions in the foreign exchange market to reducing severe short term exchange rate fluctuations. To eliminate any discrimination between market participants, the BSL replaced the temporary three auction windows in its weekly foreign exchange auction with a single auction window in September 2009.

\section{POLICIES FOR 2010}

16. The government is determined to maintain macroeconomic stability and accelerate structural reforms to set the stage for high and sustainable economic growth. To this end, the government will pursue a sustainable fiscal policy and implement reforms to improve the delivery of public services, reduce the cost of doing business, and raise productivity and employment throughout the economy.

17. A more favorable global economic environment is expected to contribute positively to Sierra Leone's macroeconomic developments in 2010. Real GDP growth is projected to increase to 4.7 percent and inflation is targeted to decline to 8 percent. Economic growth will benefit from improved supply of energy, ongoing public and private initiatives to increase agriculture productivity and intensification, and higher public investment in basic infrastructure. International reserves are expected to remain above 6 months of import coverage.

\section{Fiscal policy}

18. Fiscal policy in $\mathbf{2 0 1 0}$ will aim at consolidating macroeconomic stability and laying the basis for sustained economic growth. The proposed 2010 budget envisages 
improved revenue performance, a significant shift from current to capital spending, and a moderate reduction in the fiscal deficit.

19. On the revenue side, domestic revenue is projected to rise from 11.3 percent of GDP in 2009 to 11.6 percent of GDP. The projection reflects unchanged tax policies, including full application of policy measures adopted in the 2009 Finance Act, and efficiency gains from the modernization of the NRA, particularly the completion of ASYCUDA in customs clearing, and the introduction of the GST. The GST, which will be introduced on January 1, 2010 (structural benchmark), is expected to be revenue neutral during the first six months of the year, but to generate at least 0.1 percent of GDP during the second half. To ensure that any new revenue measures are properly incorporated into the budget prior to adoption, the 2010 Finance Act will be presented to Parliament at the same time as the 2010 budget.

Government is committed to ensuring that any new investment incentives will not negatively affect tax collections in 2010. Grants are currently projected to decline from 7.3 percent of GDP in 2009 to 7 percent of GDP. Budget support grants are projected to decline slightly to 3.7 percent of GDP.

20. On the expenditure side, the wage bill is projected to remain close to 6 percent of GDP. The projection assumes a 20 percent increase in basic pay for civil servants and the hiring of 2000 new teachers and 1000 police officers. Current nonwage, noninterest expenditures will remain at about the same relative to GDP as in 2009, excluding exceptional expenditure in 2009 for the Emergency Power Project and the government contribution to the deployment of military forces in the Dafour region. Domestically financed capital spending will be increased by about 1 percent of GDP, in line with the objective of the PRS to improve the delivery of basic social services and infrastructure, particularly roads and water distribution.

21. The domestic primary deficit is envisaged to decline from 3.8 percent of GDP in 2009 to 3.5 percent of GDP. Domestic financing will remain at 1.8 percent of GDP, including about 0.5 percent of GDP to reduce domestic arrears.

\section{Monetary and exchange rate policies}

22. Reserve money is targeted to grow by about 13 percent in $\mathbf{2 0 1 0}$. Given the government's domestic financing requirement, this would allow for adequate expansion in private sector credit and a small buildup of gross international reserves. Exchange rate flexibility will be maintained in 2010. The BSL will continue to balance treasury bill sales and foreign exchange auctions for an optimal monetary policy mix.

\section{Structural Reforms}

23. The current multiple-price Dutch foreign exchange auction, operated weekly by the BSL, was put in place in 2000 with the assistance of the IMF. The fifth review discussions brought to our attention that the Dutch auction system gives rise to multiple 
currency practices (MCPs) because there is no mechanism in place to prevent potential spreads of effective rates between the winning bids from exceeding two percent. The BSL will consult with the IMF on the best way to introduce mechanisms that would eliminate the MCP by end-June 2010.

24. The structural reform program will continue to support economic growth and improve delivery of public services. To this end, Government is committed to complete the implementation of the structural reforms envisaged in 2009. Looking further ahead, the support of our development partners will be critical to implementing the comprehensive structural reform program presented in the PRSP-II.

\section{An integrated public financial management reforms program, supported by} several development partners, is under implementation. The program seeks to consolidate the progress made so far in public expenditure management, with the aim to ensure sustainable improvement in the credibility, predictability, control and transparency of the budget. To strengthen the role of line ministries in the budget execution process and to make budget committees in these public entities fully operational, budget officers will be recruited by December 2009 and assigned to MDAs that have rolled out IFMIS (structural benchmark). The MTEF process will be deepened to ensure that future annual budget allocations are consistent with the medium-term objectives of the poverty reduction strategy.

26. Reforms in the financial sector will be actively pursued to deepen financial intermediation and ensure a strong and competitive financial sector. To this end, BSL has developed a Financial Sector Development Plan (FSDP), which has been approved by the Cabinet. The objective of the FSDP is to provide a framework for creating a sound, diversified, responsive and well-functioning financial system that would provide appropriate support to productive activities, thereby contributing to economic growth and poverty alleviation. The BSL has undertaken actions to strengthen banking supervision, particularly by increasing and training staff in the Banking Supervision Department. The BSL is seeking assistance from the IMF to revise the Banking Act and Other Financial Services Act to allow for a migration to risk-based supervision. Following the enactment of the revised acts, which is expected next year, new off-site surveillance guidelines, in line with a risk-based approach, will be issued to banks. Preparations are advancing for establishing a credit reference bureau (CRB) as well as an efficient payments system, including a Real Time Gross Settlement System (RTGS). To reduce the vulnerability from NPLs, the BSL will enforce the minimum capital requirement for all banks of Le 15 billion by end- 2009 and of Le 30 billion by end2014. The BSL Act of 2000 is under review with the intention of strengthening central bank independence.

27. Steps are being taken to accelerate the restructuring of the National Power Authority (NPA) and improve its finances. A detailed tariff study is nearly completed and it will provide inputs for a comprehensive electricity tariff policy, which will be adopted by end-December 2009 (structural benchmark). A financial controller and a billing/commercial 
manager have been recruited to improve oversight of the financial operations of the NPA. The memorandum signed between the Ministry of Finance and Economic Development and NPA for the settlement of cross debt will be reassessed to take into account the extension of the contract with the private power provider by three months and incorporate unpaid balances by the two parties.

28. Tackling corruption will remain a top priority for the government. In 2008, the government launched the national Anti-Corruption Strategy (ACS) for 2008-13 and enacted into law a revised Anti-Corruption Act that strengthens the powers of the Anti-Corruption Commission (ACC). The ACC will focus on public education and outreach, review of public service delivery in critical area, assets declarations by civil servants, and prosecution of cases of corruption. The ACC will also conduct semi-annual assessments of the national ACS to monitor progress in its implementation and take remedial actions as needed.

\section{Establishing a transparent and automatic pricing framework for petroleum} products is a priority. The current formula for petroleum pricing is frequently adjusted based on a targeted pump price and tax revenue concerns. Looking forward, domestic petroleum product prices should generally be determined on the basis of full pass-through of international prices. The government also intends to bring the Strategic Petroleum Fund (SPF) under the budget and clarify its purpose, including rules for SPF inflows and outflows.

28. The government will continue to implement reforms to improve the investment climate in order to foster sustainable economic growth. Going forward, the focus will be on increasing access to finance, improving business regulatory environment especially in areas where the country is ranked poorly in the Doing Business Report, and improving physical infrastructure.

\section{Program Monitoring}

29. The program will be monitored based on quantitative PCs for end-December 2009 (Table 1) and structural benchmarks for 2009 (Table 2). 
Table 1. Sierra Leone: Quantitative Performance Criteria and Indicative Targets for 2009

(Cumulative change from beginning of calendar year to end of month indicated; Le millions, unless otherwise indicated)

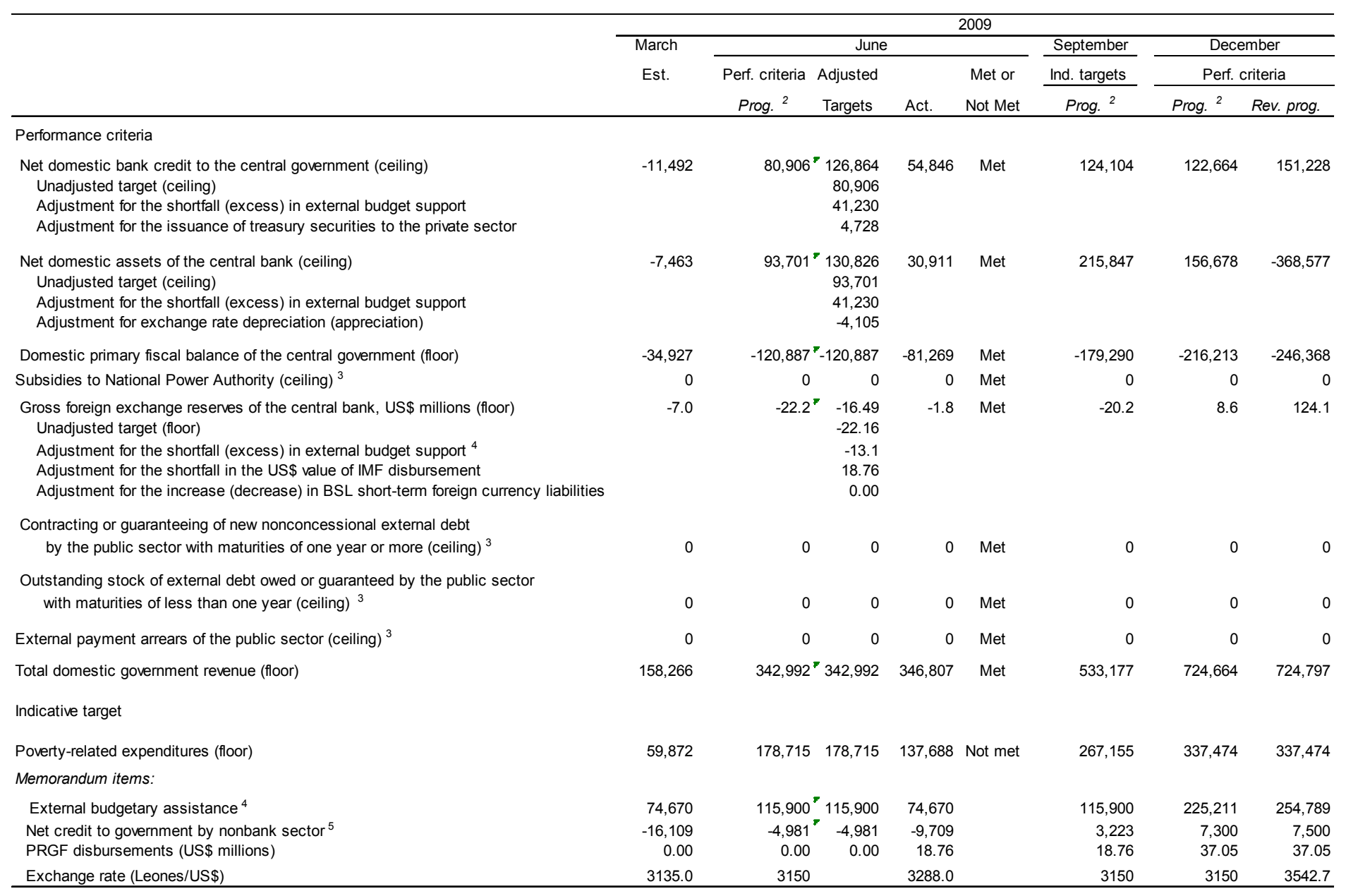

${ }^{1}$ The performance criteria and indicative targets shown in this table are defined in the Technical Memorandum of Understanding (TMU).

${ }^{2}$ IMF Country Report No. 09/215.

${ }^{3}$ These apply on a continuous basis.

${ }^{4}$ Including program grants and program loans.

${ }^{5}$ Comprises treasury bills purchased by the National Social Security and Insurance Trust (NASSIT) and the nonfinancial private sector. 
Table 2. Sierra Leone: Structural Benchmarks for 2009

Measures
Macroeconomic policy coordination
Provide to Fund staff the Monetary and
Policy Committee (MPC) monthly minutes
that include the monthly projections for
government revenue and expenditures made
available by the Ministry of Finance to the
Bank of Sierra Leone to produce a monthly
liquidity forecast.

Tax administration and policy

- Adoption by the Cabinet of the implementation decree for new and modernized customs law and regulations that reflects the WTO agreement and protects government customs revenue.

- Adoption by the Cabinet of a simplified and fully designed small taxpayer regime with supporting draft legislation.

- $\quad$ Establish a Domestic Tax Department (DTD) as the vehicle for achieving integration of domestic tax collection and make it functional.

- $\quad$ Integrate the Goods and Services Tax (GST) administration within the Large Taxpayer Office (LTO).

- Introduce the Goods and Services Tax (GST).

Public financial management

- $\quad$ Recruit and assign budget officers to MDAs that have IFMIS 'rolled out' and ensure their budget committees are fully operational.

Financial supervision

- $\quad$ Adoption by the BSL of new off-site surveillance guidelines for banks consistent with the requirements of the revised Banking and Other Financial Services Act, and introduction of new reporting requirements based on these guidelines.

Public enterprise reform

- $\quad$ Adopt a comprehensive tariff policy for the electricity sector that will strengthen the financial position of the National Power Authority.

\section{Timing Macro Rationale}

On a

continuous

basis

End-June

To enhance the collection of import duties and thus create more fiscal space to preserve priority spending.

End-

September

To improve revenue collection by extending the tax base.

\section{End-}

December

To improve efficiency of revenue collection efforts.

End-

December

To ensure full and efficient implementation of the GST to achieve domestic revenue target.

January 1 , 2010

EndDecember

Improve spending efficiency by enhancing public financial management and budget execution.

EndSeptember

Financial deepening which is vital to achieve the growth objective of the program.

EndDecember

To improve financial viability of the public electricity. This will ensure an efficient and sustainable power supply, critical for the growth objectives of the program.
Met with delay. Cabinet adopted the draft law and regulations in September.

Met. A small and medium taxpayer regime is in place. However, no new legislation nor Cabinet approval was required to implement this measure.

New measure

Not met. The new off-site guidelines could not be issued since the Banking and Other Financial Services Acts have not yet been enacted. The BSL is seeking assistance from the IMF to revise the acts. 


\section{AtTaChment II. SierRa LeONe: TeChNiCal Memorandum of Understanding}

December 7, 2009

\section{INTRODUCTION}

1. This memorandum sets out the understandings between the Sierra Leonean authorities and the International Monetary Fund (IMF) regarding the definitions of the quantitative and structural performance criteria (PCs) and benchmarks for the reminder of the program supported by the Poverty Reduction and Growth Facility (PRGF) arrangement, as well as the related reporting requirements. Unless otherwise specified, all quantitative PCs and indicative targets will be evaluated in terms of cumulative flows from the beginning of the period, as specified in Table 2 of the Memorandum of Economic and Financial Policies (MEFP) of the Government of Sierra Leone (GoSL) for 2009.

2. Program exchange rates. For the purpose of the program, foreign currency denominated transactions in the fourth quarter of 2009 will be converted from their U.S. dollar denominated value into Sierra Leonean currency (leones) using a program exchange rate of Le 3542.7/US\$.

\section{Quantitative Performance Criteria}

\section{A. Gross Foreign Exchange Reserves of the Bank of Sierra Leone (BSL)}

3. Definition. Unless otherwise noted, gross foreign exchange reserves of the Bank of Sierra Leone (BSL) are defined as reserve assets of the BSL. Reserve assets are defined in the IMF's Balance of Payments Manual ( $5^{\text {th }}$ ed.) and elaborated in the reserve template of the Fund's International Reserves and Foreign Currency Liquidity: Guidelines for a Data Template. They exclude foreign assets not readily available to, or controlled by, the monetary authorities.

\section{Adjustment clauses.}

4. The floor on the change in gross foreign exchange reserves will be adjusted (a) downward (or upward) by the amount in U.S. dollars of the shortfall (excess) in programmed external budgetary assistance - the downward adjustment will be capped at the equivalent of US\$20 million while the upward adjustment will be equal to the amount - if any, exceeding US\$20 million;' (b) downward (upward) for any shortfall (excess) in the U.S. dollar value of disbursements from the IMF under the PRGF arrangement; and (c) upward (or downward) for any increase (or decrease) in BSL short-term (one year or less in original maturity) foreign currency-denominated liabilities (to residents and nonresidents).

\footnotetext{
${ }^{1}$ External budgetary assistance is defined as program grants and program loans, excluding HIPC assistance.
} 


\section{B. Net Domestic Assets of the BSL}

5. Definition. Net domestic assets (NDA) of the BSL are defined as the end-period (based on daily data) stocks, during the month of the test dates, of the reserve money less net foreign assets calculated at the program exchange rates. Reserve money includes currency in circulation and deposits of commercial banks with the BSL. Net foreign assets of the BSL are defined as gross foreign exchange reserves (defined above) minus foreign liabilities. Foreign liabilities are defined as foreign currency-denominated liabilities of the BSL to nonresidents and the outstanding use of Fund credit. For program purposes, foreign liabilities exclude SDR allocation.

6. Adjustment clauses. The ceiling on the NDA of the BSL will be adjusted upward by the leone value of the shortfall in the external budgetary assistance at the test dates, up to a maximum of US $\$ 20$ million. In the event of an excess in the external budgetary assistance greater than US $\$ 20$ million, the NDA ceiling will be adjusted downward by the amount exceeding US\$20 million.

\section{Net Domestic Bank Credit to the Central Government (NCG)}

7. Definition. NCG refers to the net banking system's claims on the central government as calculated by the BSL. It is defined as follows:

- the net position of the government with commercial banks, including: (a) treasury bills, excluding holdings of treasury bills for monetary operations; (b) treasury bearer bonds; and (c) loan and advances of commercial banks to the government; less government deposits in commercial banks;

- $\quad$ the net position of the government with the BSL, including: (a) treasury bills, excluding holdings of treasury bills for monetary operations; (b) treasury bearer bonds; and (c) ways and means; less (a) central government deposits; and (b) HIPC and MDRI relief deposits.

8. Adjustment clauses. The ceiling on the increase in NCG will be adjusted upward (downward) by up to the leone value of the shortfall (excess) in external budgetary assistance. The upward adjustment will be capped at the equivalent of US\$20 million while the downward adjustment will be equal to the amount - if any, exceeding US\$20 million. The ceiling will also be adjusted downward (upward) by the excess (shortfall) in the leone value of net issues of government securities to the nonbank private sector vis-à-vis the program target (specified in the memorandum items in Table 2 of the MEFP).

9. Data source. The data source for the above will be the series "Claims on Government (Net)" submitted to the IMF staff and reconciled with the monthly monetary survey prepared by the BSL. These data will be reconciled with monthly reports with the monetary data (Treasury bill transactions, ways-and-means account, and Treasury bearer bond transactions).

10. Definition of Central government. Central government is defined for the purposes of this memorandum to comprise the central government and those special accounts that are classified as central government in the BSL statement of accounts. The National Social Security and Insurance Trust and public enterprises are excluded from this definition of central government. 


\section{Domestic Revenue of Central Government}

11. Definition. The floor on total domestic central government revenue is defined as total central government revenue, as presented in the central government financial operations table, excluding external grants.

\section{E. Domestic Primary Fiscal Balance of Central Government}

12. The floor on the domestic primary fiscal balance of the central government is defined as domestic revenue minus total expenditure and net lending, excluding interest payments, and externally-financed capital expenditure.

\section{F. Subsidies to the National Power Authority (NPA)}

13. Definition. The term "subsidy" refers to any government financial support (i.e., unrequited transfers) to the NPA. It does not include the government's on-lending of external loans for capital expenditure of the enterprise. The subsidy is to be reduced by the amount of arrears accumulating in regard to the charges for government's electricity consumption. This PC will apply on a continuous basis.

\section{G. External Payment Arrears of the Public Sector}

14. Definition. External payment arrears of the public sector are defined as the stock of new external overdue debt-service payments by the public sector. For the purposes of this PC, the public sector comprises the central government, regional government, all public enterprises and the BSL. The nonaccumulation of external arrears is a performance criterion during the program period. Excluded from this PC are those debts subject to rescheduling. This PC will apply on a continuous basis.

\section{H. New Nonconcessional External Debt Contracted or Guaranteed by the Public Sector with an Original Maturity of One Year or More}

15. Definition. Those are defined as all forms of new debt with original maturity of one year or more contracted or guaranteed by the public sector. ${ }^{2}$ This PC applies not only to debt as defined in point 9 of the Guidelines on Performance Criteria with Respect to Foreign Debt (Decision No. 12274 (00/85), August 24, 2000) but also to commitments contracted or guaranteed for which value has not been received. Excluded from this PC are disbursements from the IMF and those debts subject to rescheduling. For the purposes of this PC, the "public sector" is as defined in $\$ 14$ above. This $\mathrm{PC}$ will apply on a continuous basis.

\section{External Short-Term Debt Contracted or Guaranteed by the Public Sector}

16. Definition. External short-term debt is defined as external debt stock with a maturity of less than one year contracted or guaranteed by the public sector. Debt is defined in

\footnotetext{
${ }^{2}$ Debt is considered concessional if it has a grant element equivalent to 35 percent or more. Calculation of the degree of concessionality of new external borrowing is based on the last 10-year average commercial interest reference rate (CIRR) of the Organization for Economic Cooperation and Development (OECD) for loans with maturities of at least 15 years and on the last six-month average CIRR for loans maturing in less than 15 years.
} 
Annex I of this TMU. For this purpose, short-term debt will exclude normal trade credit for imports. For the purposes of this PC, the public sector is as defined in $\$ 14$ above. This PC will apply on a continuous basis.

\section{QUANTITATIVE INDICATIVE TARGET Poverty-Related Expenditures}

17. Definition. Poverty-related expenditures refer to those expenditures in the areas identified in Table 2 of the Sierra Leone HIPC Decision Point Document. These budgetary expenditures include but are not limited to those sub-components that are financed by drawdown from the MDRI Relief Account at the BSL.

\section{Program Monitoring}

18. The Sierra Leonean authorities shall maintain a program-monitoring committee composed of senior officials from the MoFED, the BSL, and other relevant agencies. The committee shall be responsible for monitoring performance under the program, recommending policy responses, informing the IMF regularly about the progress of the program, and transmitting the supporting materials necessary for the evaluation of PCs and benchmarks. In addition, the Net Domestic Financing Technical Committee shall provide the IMF with weekly minutes of its meetings complemented with the minutes of the monthly meetings of the Inter-Agency Committee for National Statistics progress report on the program on a monthly basis within four weeks of the end of each month, using the latest available data. 


\section{AnNeX 1: Implementation OF The Revised Guidelines on PeRformanCe}

\section{Criteria with Respect to Foreign Debt}

The term "debt" has the meaning set forth in point No. 9 of the Guidelines on Performance Criteria with Respect to Foreign Debt adopted on August 24, 2000, which reads as follows: "(a) For the purpose of this guideline, the term "debt" will be understood to mean a current, i.e., not contingent, liability, created under a contractual arrangement through the provision of value in the form of assets (including currency) or services, and which requires the obligor to make one or more payments in the form of assets (including currency) or services, at some future point(s) in time; these payments will discharge the principal and/or interest liabilities incurred under the contract. Debts can take a number of forms, the primary ones being as follows: (i) loans, i.e., advances of money to obligor by the lender made on the basis of an undertaking that the obligor will repay the funds in the future (including deposits, bonds, debentures, commercial loans, and buyers' credits) and temporary exchanges of assets that are equivalent to fully collateralized loans under which the obligor is required to repay the funds, and usually pay interest, by repurchasing the collateral from the buyer in the future (such as repurchase agreements and official swap arrangements); (ii) suppliers' credits, i.e., contracts where the supplier permits the obligor to defer payments until sometime after the date on which the goods are delivered or services are provided; and (iii) leases, i.e., arrangements under which property is provided which the lessee has the right to use for one or more specified period(s) of time that are usually shorter than the total expected service life of the property, while the lessor retains the title to the property. For the purpose of the guideline, the debt is the present value (at the inception of the lease) of all lease payments expected to be made during the period of the agreement excluding those payments that cover the operation, repair, or maintenance of the property. (b) Under the definition of debt set out above, arrears, penalties, and judicially awarded damages arising from the failure to make payment under a contractual obligation that constitutes debt are debt. Failure to make payment on an obligation that is not considered debt under this definition (e.g., payment on delivery) will not give rise to debt". (c) Excluded from this performance criterion are normal import-related credits, disbursements from the IMF, and those debts subject to rescheduling arrangements." 


\section{Sierra Leone: Summary of Data to be Reported to IMF Staff}

\begin{tabular}{|c|c|c|c|}
\hline Type of Data & Tables & Frequency & Reporting Deadline \\
\hline \multirow[t]{3}{*}{ Real sector } & National accounts & Annual & End of year +9 months \\
\hline & Revisions of national accounts & Variable & End of revision +2 months \\
\hline & Disaggregated consumer price index & Monthly & End of month +2 weeks \\
\hline \multirow[t]{5}{*}{ Public finance } & $\begin{array}{l}\text { Net government position and details of } \\
\text { nonbank financing, including the stock of } \\
\text { the float, treasury bills, and bonds, as well } \\
\text { as privatization receipts }\end{array}$ & Monthly & End of month +6 weeks \\
\hline & $\begin{array}{l}\text { Government flow-of-funds table } \\
\text { (Government Financial Operations Table) } \\
\text { with supporting documentation (final) and } \\
\text { presented on commitment and cash bases }\end{array}$ & Monthly & End of month +6 weeks \\
\hline & $\begin{array}{l}\text { Petroleum product pricing formula, tax } \\
\text { receipts by categories of petroleum } \\
\text { products }\end{array}$ & Monthly & End of month +6 weeks \\
\hline & $\begin{array}{l}\text { Stock of outstanding payment commitments } \\
\text { with a breakdown between current and } \\
\text { capital expenditures }\end{array}$ & Monthly & End of month +6 weeks \\
\hline & $\begin{array}{l}\text { Import duty exemptions by end-users and } \\
\text { tariff regimes and estimates of } \\
\text { corresponding revenue losses }\end{array}$ & Quarterly & End of quarter +6 weeks \\
\hline \multirow[t]{9}{*}{$\begin{array}{l}\text { Monetary and financial } \\
\text { data }\end{array}$} & Monetary survey & Monthly & End of month +6 weeks \\
\hline & Balance sheet of the BSL & Monthly & End of month +6 weeks \\
\hline & $\begin{array}{l}\text { Consolidated balance sheets of commercial } \\
\text { banks }\end{array}$ & Monthly & End of month +6 weeks \\
\hline & $\begin{array}{l}\text { BSL monitoring sheet of net financing of the } \\
\text { financial sector to the government }\end{array}$ & Monthly & End of month +6 weeks \\
\hline & $\begin{array}{l}\text { BSL monitoring sheet of treasury bills and } \\
\text { bonds holdings }\end{array}$ & Monthly & End of month +6 weeks \\
\hline & Borrowing and lending interest rates & Monthly & End of month +6 weeks \\
\hline & $\begin{array}{l}\text { Results of foreign exchange and Treasury } \\
\text { Bills auctions }\end{array}$ & Weekly & End of week +3 days \\
\hline & Stocks of government securities & Monthly & End of month +6 weeks \\
\hline & Banking supervision ratios & Quarterly & End of quarter +8 weeks \\
\hline
\end{tabular}


Sierra Leone: Summary of Data to Be Reported to IMF Staff (concluded)

\begin{tabular}{|c|c|c|c|}
\hline Type of Data & Tables & Frequency & Reporting Deadline \\
\hline \multicolumn{4}{|l|}{$\begin{array}{l}\text { Monetary and financial } \\
\text { data }\end{array}$} \\
\hline & Gross official foreign reserves & Weekly & End of week + 1 week \\
\hline & Foreign exchange cashflow table & Quarterly & End of quarter +4 weeks \\
\hline & Revised balance of payments data & Variable & When revisions occur \\
\hline & $\begin{array}{l}\text { Exports and imports of goods (including the } \\
\text { volume of key minerals and fuels) }\end{array}$ & Monthly & End of month +3 months \\
\hline \multirow[t]{3}{*}{ External debt } & $\begin{array}{l}\text { Outstanding external arrears and } \\
\text { repayments (if applicable) }\end{array}$ & Monthly & End of month +4 weeks \\
\hline & $\begin{array}{l}\text { Details of all new external borrowing and } \\
\text { guarantees provided by government on } \\
\text { new borrowing }\end{array}$ & Monthly & End of month +4 weeks \\
\hline & $\begin{array}{l}\text { External debt service payments (separately } \\
\text { on principal and interest payment) to each } \\
\text { creditor }\end{array}$ & Monthly & End of month +4 weeks \\
\hline \multirow[t]{4}{*}{$\begin{array}{l}\text { HIPC initiative and MDRI } \\
\text { monitoring }\end{array}$} & $\begin{array}{l}\text { Statement of special account at the BSL, } \\
\text { that receives resources generated by the } \\
\text { HIPC Initiative and tracks their use }\end{array}$ & Monthly & End of month +4 weeks \\
\hline & $\begin{array}{l}\text { Statement of special MDRI account at the } \\
\text { BSL and the corresponding poverty- } \\
\text { reducing spending financed }\end{array}$ & Monthly & End of month +4 weeks \\
\hline & $\begin{array}{l}\text { Minutes of the meeting of the Net Domestic } \\
\text { Financing (NDF) Technical Committee }\end{array}$ & Weekly & End of meeting +2 weeks \\
\hline & $\begin{array}{l}\text { Minutes of the meeting of the Monetary } \\
\text { Policy Committee }\end{array}$ & Monthly & Date of meeting +2 weeks \\
\hline
\end{tabular}




\title{
INTERNATIONAL MONETARY FUND AND \\ INTERNATIONAL DEVELOPMENT ASSOCIATION
}

\author{
SIERRA LEONE \\ Joint IMF/World Bank Debt Sustainability Analysis 2009 \\ Prepared by the staffs of the International Monetary Fund and \\ the International Development Association
}

Approved by Seán Nolan and Dominique Desruelle (IMF)

and Carlos Primo Braga and Sudhir Shetty (World Bank)

December 7, 2009

This debt sustainability analysis for low-income countries (LIC-DSA) is based on end-2008 data for external and domestic debt provided by the Sierra Leonean authorities. Staff's assessment is that Sierra Leone's risk of debt distress remains moderate. Under baseline projections, all external debt indicators are below their indicative thresholds throughout the projection period (2009-29). However, external debt ratios move toward the thresholds or breach them under less favorable scenarios and stress tests. Public sector debt dynamics remain on a stable path under the baseline scenario. The analysis highlights the continued need for improved domestic revenue mobilization, the containment of low priority current expenditures, and growth boosting and export-enhancing policies and programs as stress tests suggest that potential threats to sustainability remain. Moreover, Sierra Leone should continue to contract new external financing only in the form of grants and highly concessional loans and promote the development of a domestic debt market.

\section{BACKGROUND}

1. This debt sustainability analysis (DSA) updates the DSA presented in June 2008 (IMF Country Report, No. 08/249). This Low Income Country (LIC)-DSA update, jointly conducted by the Fund and World Bank staffs in collaboration with the authorities, is based on estimated outstanding stock of external and domestic debt at end-2008 and projected debt service due in 2009-55. Projected debt service to the World Bank, the African Development Bank and the IMF was estimated by staff of these institutions. Projected debt service due to other external creditors was obtained from the authorities. Debt service due on domestic debt was provided by the authorities for the period $2009-10$ and projected by the staffs for the subsequent years. The macroeconomic framework underlying this DSA was prepared by the staffs, based on the outcome of the fifth review under the PRGF-supported program.

2. Sierra Leone reached the completion point under the enhanced HIPC Initiative and qualified for debt relief under the MDRI on December 15, 2006. In January 2007, Paris Club creditors agreed to cancel outstanding claims (US\$240 million in end-2000 PV terms) on 
Sierra Leone. ${ }^{7}$ Debt relief from the international community helped decrease Sierra Leone's public sector nominal external debt from about 142 percent of GDP at end-2005 to about 32 percent of GDP at end-2007.

3. At end-2008, Sierra Leone's nominal public and publicly guaranteed external debt, including arrears, was estimated at US\$620.2 million (33 percent of GDP). Half of this debt was due to multilaterals, 39 percent to commercial creditors, and the remainder to official bilateral creditors. ${ }^{8}$ The largest multilateral creditors were the World Bank Group (US\$109 million), the IMF (US\$53.5 million), the Islamic Development Bank (US\$43.8 million) and the African Development Bank (US\$43.3 million). All external debts to commercial creditors were mostly arrears accumulated before and during the civil war, which ended in 2002. The Sierra Leone government continues to make goodwill payments to some of the commercial creditors to avoid litigation. ${ }^{9}$ A debt-buy-back operation is under preparation, with World Bank assistance, to cancel all eligible commercial debt by end-2011. ${ }^{10}$

\section{Domestic debt, including verified domestic arrears, amounted to 21 percent of GDP} at end-2008. Around 41 percent of this debt was with the central bank of Sierra Leone, including a large stock of non-interest bearing securities. Commercial banks and other financial institutions accounted for another 52 percent. Arrears to the non-financial private sector constituted the remaining 7 percent of domestic debt. Interest payments on domestic debt in 2008 represented 16 percent of domestic revenue and 13 percent of current expenditure.

\section{UNDERLYING DSA ASSUMPTIONS}

5. The macroeconomic framework underlying this DSA differs from the one presented in the June 2008 DSA on the starting point as well as on certain critical assumptions. Since the completion of the previous DSA, the Sierra Leonean economy has been adversely affected by the global economic downturn, which resulted in a slower economic growth and a steep decline in exports. In addition, unanticipated technical difficulties have crippled two major mining companies and anticipated foreign direct investment in the mining sector is likely to be postponed. As a result, actual exports in 2008 are significantly lower than projected in the previous DSA. Although a pick-up in exports is expected, the revised macroeconomic framework remains cautious with the ratio of exports-to-GDP staying below the ratio projected

\footnotetext{
${ }^{7}$ Sierra Leone has received debt relief under HIPC and MDRI Initiatives from the IMF, IDA, AfDB, EIB, IFAD, BADEA, IDB, and OPEC Fund. Bilateral agreements have been signed with all Paris Club creditors. Agreements on the delivery of the HIPC relief are still pending with China, Kuwait, and Saudi Arabia.

${ }^{8}$ At present, all external loans contracted by state-owned enterprises are guaranteed by the government.

${ }^{9}$ A lawsuit against Sierra Leone by one commercial creditor is still ongoing.

${ }^{10}$ Unreconciled records suggest that the stock of external arrears to commercial creditors could be as high as US\$243 million at end-2008. In February 2009, the World Bank's Board approved a grant to fund the preparatory work for the operation, including for legal and financial advisors and audits. The preparation and implementation are estimated to take about one year each.
} 
in the previous DSA. Data provided by the authorities indicates that the stock of actual external debt in 2008 is higher than projected in the previous DSA, in part due to higher than anticipated new borrowing. A large part of the additional borrowing in 2008, as well as over 2009-10, comes from the increase in access to Fund financing, which was obtained in December 2008 and June 2009 to mitigate the impact of the global downturn on Sierra Leone's international reserves. The global economic downturn is also expected to lead to a lower domestic government revenue to GDP ratio than projected in the previous DSA. The baseline macroeconomic assumptions underlying this DSA are summarized in Box 1.

\begin{tabular}{|c|c|c|c|c|}
\hline \multicolumn{5}{|c|}{ Comparison of Key Macrovariables in the New and the Previous DSA } \\
\hline & \multicolumn{2}{|c|}{2008} & \multicolumn{2}{|c|}{$\begin{array}{c}\text { Annual Average over 2009-13 } \\
\text { (unless otherwise indicated) }\end{array}$} \\
\hline & $\begin{array}{l}2008 \text { DSA } \\
\text { Projections }\end{array}$ & $\begin{array}{c}\text { New DSA } \\
\text { Actual }\end{array}$ & $\begin{array}{l}2008 \text { DSA } \\
\text { Projections }\end{array}$ & $\begin{array}{l}\text { New DSA } \\
\text { Projections }\end{array}$ \\
\hline Stock of external debt, end-of-period (US\$ million) & 538.6 & 608.4 & 657.8 & 727.3 \\
\hline Total new external borrowing (US\$ million) & 46.8 & 72.8 & 77.3 & 84.3 \\
\hline Debt service on external debt (US\$ million) & 11.9 & 10.6 & 15.2 & 21.3 \\
\hline Real GDP growth (percent) & 6.0 & 5.5 & 5.5 & 5.3 \\
\hline Exports of Goods and nonfactor services (percent of GDP) & 20.6 & 16.3 & 27.1 & 18.2 \\
\hline Current account deficit (percent of GDP) & 6.5 & 10.7 & 5.6 & 7.9 \\
\hline Domestic government revenue (percent of GDP) & 11.9 & 11.4 & 13.4 & 12.5 \\
\hline Domestic debt (percent of GDP) & 22.1 & 22.0 & 18.1 & 15.0 \\
\hline
\end{tabular}




\section{Box 1: Baseline Macroeconomic Assumptions Underlying the DSA}

- $\quad$ Real GDP growth reached 5.5 percent in 2008 but is projected to slow to 4 percent in 2009 . With a strengthening of the external environment since the mid-2009, including for diamond prices, the outlook for the Sierra Leonean economy is beginning to improve. Economic activity is projected to gradually recover to 6.5 in 2013 and grow at a steady rate of 5 percent after 2015. Medium to long-term growth is predicated on government's ongoing policies to consolidate macroeconomic stabilization, expand basic public infrastructure, and improve the business environment for private sector development.

- $\quad$ Average annual inflation, as measured by the CPI, is expected to decline from its peak in 2008 of about 15 percent to slightly below 10 percent in 2009 and then gradually to 5 percent by 2015 and 4 percent by 2029. The projection reflects the WEO assumptions on the prices of the main commodities, as well as the authorities' commitment to refrain from central bank financing and to strengthen central bank capacity in conducting monetary policy.

- $\quad$ Exports are projected to benefit from a projected increase in commodity prices, expansion in mining capacity, and increased investment in agriculture. The new DSA assumes a steeper upward trend in exports than the previous DSA but without attaining the export-to-GDP ratio that was previously anticipated. Total exports are expected to gradually increase from about 16 percent of GDP in 2009 to 31 percent by 2029. Imports of goods and services are projected to gradually increase from about 29 percent in 2009 to 41 percent in 2029.

- $\quad$ On the fiscal side, the planned introduction of a value added tax in January 2010, together with ongoing strengthening and modernization of customs and tax administration is expected to gradually broaden the tax base, raising domestic revenue from 11.4 percent of GDP in 2008 to 13.4 percent in 2013 and gradually to 16 percent by 2029 . Current expenditures are projected to remain around 14 percent, while public capital expenditures are expected to increase from 6 percent of GDP in 2008 to 9 percent by 2012 and 11 percent by 2029 in order to address the substantial infrastructure needs of the country. The overall fiscal deficit, including grants, is projected to gradually decrease from 4.8 percent of GDP in 2008 to 2.5 percent by 2029 .

- Donor assistance, including grants and concessional loans, is expected to average 10 percent of GDP in 2009-13 and to decline to an annual average of 9 percent in 2014-20 and 8 percent in 2021-29.

- No new debt operation is assumed beyond the buy-back of the external commercial debts that is expected to take place in 2011. It is assumed that about US\$184 million of commercial debt would be eligible for the debt buy-back operation ( 80 percent of the estimated total) and that 90 percent of that amount would be cancelled. Out of the 10 percent remaining, 75 percent will be covered by external grants and the remaining 25 percent will have to be financed by the budget.

- Domestic debt is expected to decline gradually from 22 percent of GDP in 2008 to 8 percent in 2029 as the government refrains from central bank borrowing and limits issuance of new securities. It is assumed that domestic accumulated arrears to local suppliers and parastatals will be cleared by 2013. The remaining stock of non-interest bearing securities held by the central bank will be converted into interest bearing securities over the next ten years. A more aggressive schedule would be costly and could induce the need for more borrowing. 


\section{EXTERnAL DEBT SuStainabiLITY}

\section{A. Baseline}

6. Under the baseline scenario, the debt indicators are projected to remain below the corresponding thresholds throughout the entire projection period. ${ }^{11}$ The $\mathrm{PV}$ of debt-toexport ratio is projected to be at its highest level in 2009 as the export sector has been affected by a fall in export prices, particularly for diamond and bauxite and technical difficulties in the production of diamond and rutile. In recent months, however, there have been signs of a pick-up in exports of certain products, including agricultural and diamonds. This starting trend is expected to continue as the global economy recovers, foreign direct investments rebound, and ongoing initiatives to improve agriculture productivity take hold. The PV of external debt to exports is projected to decline from 97.6 percent in 2009 to 77.2 percent in 2019 and 59.8 percent in 2029, thus remaining below the threshold of 100 percent. The PV of debt-to-GDP ratio would remain in the range of 15-19 percent during the entire projection period, thus below the threshold of 30 percent. In addition, the debt service-to exports ratio would stay under 6 percent of exports under the baseline scenario, well below the 15 percent threshold.

\section{B. Alternative Scenarios and Stress Tests}

\section{The alternative scenarios highlight the need for maintaining prudent external debt} management and refraining from non-concessional borrowing. Under the alternative scenario that assumes external new borrowing on less concessional terms (A.2), the PV of debtto-exports ratio exceeds the indicative thresholds during $2012-23 .{ }^{12}$ It would therefore be important to pursue a prudent external debt management policy relying mostly on grants and highly concessional loans.

\section{The use of the new SDR allocation would have an impact on Sierra Leone's debt} burden, increasing the PV of external debt to exports above the threshold until 2016. The combined new allocation for Sierra Leone was SDR82.1 million, equivalent to about 6.6 percent of GDP and 42 percent of exports; therefore the interest obligations associated with the full use of the new allocation could have an important bearing on the country's debt sustainability. ${ }^{13}$ The baseline scenario assumes no use of the new SDR allocation since the authorities have not yet decided how and when they will use it. Under an alternative extreme scenario that assumes the

\footnotetext{
${ }^{11}$ Sierra Leone remains rated as a poor performer with regard to its policies and institutions with an average 2006-08 Country Policy and Institutional Assessment (CPIA) rating of 3.10. As a poor performer, the debt and debt service thresholds under the joint IMF-WB DSA framework for LICs applied to Sierra Leone are: (i) 100 percent for Present Value (PV) of debt-to-exports, (ii) 30 percent for PV of debt-to-GDP, and (iii) 200 percent for PV of debt-to-revenue. The relevant debt service thresholds are (i) 15 percent of exports, and (ii) 25 percent of revenues.

${ }^{12}$ This scenario assumes that the interest rate on new borrowing is 200 basis points higher than in the baseline scenario, while grace and maturity are the same as in the baseline.

${ }^{13}$ The LIC-DSA projects SDR interest rate to gradually increase from 0.46 percent in 2009 to 3.72 percent in 2013 and to remain at that level thereafter.
} 
total amount of new SDR allocation is used over 2011-15 in equal annual amounts, the PV of external debt to exports would remain above the 100 percent indicative threshold during 2013-16.

9. The analysis also shows that the debt-to-export ratio is very sensitive to export shocks. Under the most extreme bound test where the value of exports grows at historical average minus one standard deviation in 2010-11 (B2), the PV of debt-to-exports ratio would reach 174 percent of exports in 2014 and gradually decline to 100 percent in $2028 .{ }^{14}$ The large average deviation to the threshold amounting to about 36 percentage points underlines the vulnerability of the economy to adverse external developments. This calls for strengthening the environment for growth boosting and export expanding policies and programs, including continuing infrastructure investment and financial deepening.

\section{Fiscal DebT Sustainability}

\section{A. Baseline}

10. Under the baseline, Sierra Leone's total public debt burden (including domestic debt) is expected to stabilize over the projection period. The baseline macroeconomic scenario assumes a gradual reduction in domestic bank financing, as a result of the projected fiscal consolidation. With moderate domestic financing, domestic debt is expected to decline from 21 percent of GDP in 2008 to 8 percent by 2029. This trend is offsetting the mild increase in external debt, so that the PV of total public debt-to-GDP ratio would decline from 34 percent of GDP in 2008 to 27 percent in 2029 (Table 3a). While a lower accumulation of domestic debt is a positive outcome, there is still a need to develop a more competitive domestic debt market that could result in lower interest rates and longer maturities.

\section{B. Alternative Scenarios and Stress Tests}

\section{A high primary fiscal deficit is the most unfavorable stress test for public debt} dynamics. Under a scenario that assumes the primary fiscal deficit to remain at its 2009 level throughout the projection period, the PV of debt-to-GDP ratio would be the highest among those generated in the three alternative scenarios considered. This underscores the importance of improving domestic revenue mobilization and containing non-priority current expenditures.

\section{DebT Distress Classification and Conclusions}

\section{Based on the LIC-DSA framework, Sierra Leone remains at a moderate risk of} external debt distress. Under the baseline scenario, all debt indicators are below the indicative country-specific policy dependent thresholds. However, stress tests reveal that Sierra Leone's external debt trajectory is still vulnerable to shocks affecting its external sector. Debt burden

\footnotetext{
${ }^{14}$ Due to large fluctuations in the economic data following the end of the civil war in 2002, stress tests have been calibrated to use a 5-year historical period.
} 
indicators rise rapidly above the indicative thresholds in case the growth of exports is below its five year historical average. This illustrates the extent to which the evolution of external debt critically hinges on policies aimed at boosting growth and diversifying the export base, while continuing to access grants and highly concessional loans.

13. With the public domestic debt projected to decline, the overall assessment does not change once domestic debt is included in the analysis. A slowdown in domestic debt accumulation would, however, lessen liquidity and the rollover risks associated with the short maturities of domestic debt. Policies should aim, therefore, at promoting the development of the domestic debt market. In addition, the stress test based on a larger primary deficit underlines the importance of improving domestic revenue mobilization and containing non-priority expenditures. 
Table 1a.: External Debt Sustainability Framework, Baseline Scenario, 2006-2029 1/ (In percent of GDP, unless otherwise indicated)

\begin{tabular}{|c|c|c|c|c|c|c|c|c|c|c|c|c|c|c|c|}
\hline & \multicolumn{3}{|c|}{ Actual } & \multirow{2}{*}{$\begin{array}{l}\text { Historical } \\
\text { Average }\end{array}$} & \multirow{2}{*}{$\begin{array}{l}\text { Standard } \\
\text { Deviation }\end{array}$} & \multicolumn{10}{|c|}{ Projections } \\
\hline & 2006 & 2007 & 2008 & & & 2009 & 2010 & 2011 & 2012 & 2013 & 2014 & $\begin{array}{c}\begin{array}{c}2009-2014 \\
\text { Average }\end{array} \\
\end{array}$ & 2019 & 2029 & $\begin{array}{c}\text { 2015-2029 } \\
\text { Average }\end{array}$ \\
\hline External debt (nominal) 1/ & 110.4 & 31.7 & 32.9 & & & 39.3 & 39.4 & 28.0 & 28.8 & 29.5 & 29.8 & & 28.9 & 27.3 & \\
\hline o/w public and publicly guaranteed (PPG) & 110.4 & 31.7 & 32.9 & & & 39.3 & 39.4 & 28.0 & 28.8 & 29.5 & 29.8 & & 28.9 & 27.3 & \\
\hline Change in external debt & -33.2 & -78.7 & 1.2 & & & 6.4 & 0.1 & -11.4 & 0.8 & 0.7 & 0.3 & & -0.2 & -0.3 & \\
\hline Identified net debt-creating flows & -16.7 & -14.5 & 4.0 & & & 6.9 & 5.8 & 4.4 & 4.4 & 3.9 & 3.8 & & 2.9 & 2.9 & \\
\hline Non-interest current account deficit & 1.7 & 3.1 & 10.4 & 4.8 & 3.4 & 9.2 & 8.4 & 7.3 & 7.0 & 6.6 & 6.2 & & 5.1 & 4.9 & 5.0 \\
\hline Deficit in balance of goods and services & 7.6 & 7.0 & 13.0 & & & 12.8 & 13.0 & 12.1 & 11.7 & 11.3 & 10.7 & & 9.5 & 9.6 & \\
\hline Exports & 24.9 & 20.8 & 16.3 & & & 15.7 & 17.9 & 19.0 & 19.2 & 19.4 & 19.6 & & 23.4 & 31.0 & \\
\hline Imports & 32.5 & 27.8 & 29.4 & & & 28.5 & 31.0 & 31.0 & 30.9 & 30.7 & 30.3 & & 32.8 & 40.6 & \\
\hline Net current transfers (negative $=$ inflow) & -8.2 & -5.7 & -4.4 & -8.0 & 3.0 & -5.6 & -6.5 & -6.5 & -6.3 & -6.2 & -5.9 & & -5.5 & -5.5 & -5.6 \\
\hline $\mathrm{o} / \mathrm{w}$ official & -5.3 & -3.5 & -2.5 & -0.0 & 3.0 & -3.8 & -3.7 & -3.7 & -3.5 & -3.4 & -2.9 & & -2.4 & -1.8 & -5.0 \\
\hline Other current account flows (negative $=$ net inflow) & 2.3 & 1.8 & 1.8 & & & 1.9 & 1.8 & 1.7 & 1.6 & 1.5 & 1.4 & & 1.1 & 0.8 & \\
\hline Net FDI (negative $=$ inflow) & -2.0 & -2.0 & -2.1 & -2.1 & 0.1 & -1.3 & -1.3 & -1.2 & -1.2 & -1.2 & -1.2 & & -1.1 & -0.9 & -1.0 \\
\hline Endogenous debt dynamics $2 /$ & -16.4 & -15.7 & -4.4 & & & -1.0 & -1.3 & -1.7 & -1.3 & -1.5 & -1.3 & & -1.1 & -1.1 & \\
\hline Contribution from nominal interest rate & 2.0 & 0.4 & 0.4 & & & 0.3 & 0.4 & 0.3 & 0.2 & 0.2 & 0.3 & & 0.3 & 0.2 & \\
\hline Contribution from real GDP growth & -9.1 & -6.1 & -1.5 & & & -1.3 & -1.8 & -2.0 & -1.5 & -1.8 & -1.5 & & -1.4 & -1.3 & \\
\hline Contribution from price and exchange rate changes & -9.3 & -10.0 & -3.2 & & & & & & & & & & & & \\
\hline Residual (3-4) 3/4/ & -16.5 & -64.2 & -2.8 & & & -0.6 & -5.7 & -15.8 & -3.6 & -3.2 & -3.5 & & -3.1 & -3.2 & \\
\hline $\mathrm{o} / \mathrm{w}$ exceptional financing & -10.2 & -1.9 & 1.2 & & & -4.5 & 0.2 & -0.5 & -0.7 & -0.8 & -0.7 & & -0.3 & -0.4 & \\
\hline PV of external debt $5 /$ & $\ldots$ & $\ldots$ & 11.8 & & & 15.3 & 16.0 & 16.1 & 16.9 & 17.6 & 18.0 & & 18.1 & 18.5 & \\
\hline In percent of exports & ... & $\ldots$ & 71.9 & & & 97.6 & 89.0 & 84.9 & 88.4 & 90.7 & 91.6 & & 77.2 & 59.8 & \\
\hline PV of PPG external debt & ... & ... & 11.8 & & & 15.3 & 16.0 & 16.1 & 16.9 & 17.6 & 18.0 & & 18.1 & 18.5 & \\
\hline In percent of exports & $\ldots$ & $\ldots$ & 71.9 & & & 97.6 & 89.0 & 84.9 & 88.4 & 90.7 & 91.6 & & 77.2 & 59.8 & \\
\hline In percent of government revenues & $\ldots$ & $\ldots$ & 102.8 & & & 134.2 & 137.1 & 124.7 & 127.6 & 130.1 & 129.4 & & 121.1 & 112.6 & \\
\hline Debt service-to-exports ratio (in percent) & 18.3 & 7.8 & 3.3 & & & 4.6 & 4.7 & 5.4 & 4.6 & 4.3 & 4.7 & & 4.7 & 3.5 & \\
\hline PPG debt service-to-exports ratio (in percent) & 18.3 & 7.8 & 3.3 & & & 4.6 & 4.7 & 5.4 & 4.6 & 4.3 & 4.7 & & 4.7 & 3.5 & \\
\hline PPG debt service-to-revenue ratio (in percent) & 38.5 & 14.8 & 4.8 & & & 6.3 & 7.2 & 8.0 & 6.7 & 6.2 & 6.6 & & 7.4 & 6.6 & \\
\hline Total gross financing need (Billions of U.S. dollars) & 0.3 & 0.3 & 0.4 & & & 0.4 & 0.4 & 0.4 & 0.2 & 0.2 & 0.2 & & 0.2 & 0.3 & \\
\hline Non-interest current account deficit that stabilizes debt ratio & 34.9 & 81.8 & 9.2 & & & 2.8 & 8.3 & 18.7 & 6.2 & 5.8 & 5.9 & & 5.3 & 5.2 & \\
\hline \multicolumn{16}{|l|}{ Key macroeconomic assumptions } \\
\hline Real GDP growth (in percent) & 7.3 & 6.4 & 5.5 & 6.8 & 0.8 & 4.0 & 4.7 & 5.5 & 6.0 & 6.5 & 5.5 & 5.4 & 5.0 & 5.0 & 5.0 \\
\hline GDP deflator in US dollar terms (change in percent) & 6.9 & 9.9 & 11.3 & 7.3 & 3.3 & -4.5 & -2.3 & 3.2 & 2.4 & 0.4 & 0.4 & -0.1 & 1.7 & 1.2 & 1.4 \\
\hline Effective interest rate (percent) $6 /$ & 1.6 & 0.4 & 1.3 & 1.3 & 0.5 & 0.9 & 1.2 & 0.9 & 0.9 & 0.9 & 0.9 & 0.9 & 1.0 & 1.0 & 1.0 \\
\hline Growth of exports of G\&S (US dollar terms, in percent) & 21.4 & -2.6 & -7.6 & 7.4 & 12.6 & -4.7 & 16.9 & 15.3 & 9.5 & 8.4 & 7.0 & 8.8 & 10.5 & 9.0 & 9.8 \\
\hline Growth of imports of G\&S (US dollar terms, in percent) & 2.3 & -0.1 & 24.2 & 8.1 & 15.0 & -3.5 & 11.0 & 9.2 & 7.9 & 6.5 & 4.4 & 5.9 & 8.7 & 8.5 & 8.6 \\
\hline Grant element of new public sector borrowing (in percent) & & & & & $\ldots$ & 35.3 & 41.0 & 42.1 & 42.8 & 42.1 & 42.9 & 41.0 & 41.1 & 38.9 & 40.0 \\
\hline Government revenues (excluding grants, in percent of GDP) & 11.8 & 10.9 & 11.4 & & & 11.4 & 11.6 & 12.9 & 13.3 & 13.5 & 13.9 & 41.0 & 14.9 & 16.4 & 15.4 \\
\hline Aid flows (in Billions of US dollars) $8 /$ & 0.1 & 0.1 & 0.1 & & & 0.2 & 0.2 & 0.2 & 0.2 & 0.2 & 0.3 & & 0.3 & 0.6 & \\
\hline $\mathrm{o} / \mathrm{w}$ Grants & 0.1 & 0.1 & 0.1 & & & 0.1 & 0.1 & 0.1 & 0.2 & 0.2 & 0.2 & & 0.2 & 0.4 & \\
\hline $\mathrm{o} / \mathrm{w}$ Concessional loans & 0.0 & 0.0 & 0.1 & & & 0.1 & 0.1 & 0.1 & 0.1 & 0.1 & 0.1 & & 0.1 & 0.1 & \\
\hline Grant-equivalent financing (in percent of GDP) 9/ & & & $\ldots$ & & & 9.2 & 8.4 & 8.1 & 8.0 & 7.7 & 7.9 & & 7.2 & 6.9 & 7.0 \\
\hline Grant-equivalent financing (in percent of external financing) $9 /$ & & & & & & 73.0 & 79.8 & 79.1 & 79.2 & 80.3 & 84.3 & & 83.1 & 84.0 & 82.9 \\
\hline \multicolumn{16}{|l|}{ Memorandum items: } \\
\hline Nominal GDP (Billions of US dollars) & 1.4 & 1.7 & 2.0 & & & 1.9 & 2.0 & 2.2 & 2.3 & 2.5 & 2.7 & & 3.6 & 6.8 & \\
\hline Nominal dollar GDP growth & 14.7 & 17.0 & 17.5 & & & -0.6 & 2.2 & 8.9 & 8.6 & 7.0 & 5.9 & 5.3 & 6.8 & 6.3 & 6.5 \\
\hline PV of PPG external debt (in Billions of US dollars) & & & 0.2 & & & 0.3 & 0.3 & 0.3 & 0.4 & 0.4 & 0.5 & & 0.7 & 1.3 & \\
\hline (PVt-PVt-1)/GDPt-1 (in percent) & & & & & & 2.7 & 1.7 & 1.6 & 2.1 & 1.9 & 1.4 & 1.9 & 1.2 & 1.0 & 1.2 \\
\hline
\end{tabular}

Sources: Country authorities; and staff estimates and projections.

$1 /$ Includes both public and private sector external debt.

2/ Derived as $[r-g-\rho(1+g)](1+g+\rho+g \rho)$ times previous period debt ratio, with $r=$ nominal interest rate; $g=$ real GDP growth rate, and $\rho=$ growth rate of GDP deflator in U.S. dollar terms.

The large residid

5/ Assumes that PV of private sector debt is equivalent to its face value.

7/ Historical averages and standard deviations are derived over the past 5 years. A longer period is not considered due to large fluctuations in the post-conflict economic data.

$8 /$ Defined as grants, concessional loans, and debt relief.

9/ Grant-equivalent financing includes grants provided directly to the government and through new borrowing (difference between the face value and the PV of new debt). 
Table 2.a. Sierra Leone: Sensitivity Analysis for Key Indicators of Public and Publicly Guaranteed External Debt, 2009-2029 (In percent)

\begin{tabular}{|c|c|c|c|c|c|c|c|c|}
\hline & \multicolumn{8}{|c|}{ Projections } \\
\hline & 2009 & 2010 & 2011 & 2012 & 2013 & 2014 & 2019 & 2029 \\
\hline Baseline & 15.3 & 16.0 & 16.1 & 16.9 & 17.6 & 18.0 & 18.1 & 18.5 \\
\hline \multicolumn{9}{|l|}{ A. Alternative Scenarios } \\
\hline A1. Key variables at their historical averages in 2009-2029 1/ & 15.3 & 11.3 & 8.1 & 6.9 & 5.9 & 4.7 & 2.1 & 2.3 \\
\hline A2. New public sector loans on less favorable terms in 2009-2029 2/ & 15.3 & 16.7 & 17.8 & 19.5 & 20.9 & 21.9 & 24.8 & 28.6 \\
\hline A3. Total new SDR allocation is used over 2011-15 in an equal annual amount & 15.3 & 16.0 & 17.2 & 19.0 & 20.5 & 21.6 & 21.3 & 20.3 \\
\hline \multicolumn{9}{|l|}{ B. Bound Tests } \\
\hline B1. Real GDP growth at historical average minus one standard deviation in 2010-2011 & 15.3 & 15.4 & 15.4 & 16.2 & 16.8 & 17.2 & 17.5 & 18.1 \\
\hline B2. Export value growth at historical average minus one standard deviation in 2010-2011 3/ & 15.3 & 17.7 & 21.5 & 22.0 & 22.5 & 22.8 & 21.6 & 19.8 \\
\hline B3. US dollar GDP deflator at historical average minus one standard deviation in 2010-2011 & 15.3 & 14.7 & 14.6 & 15.3 & 16.0 & 16.3 & 16.6 & 17.2 \\
\hline B5. Combination of B1-B4 using one-half standard deviation shocks & 15.3 & 14.6 & 15.6 & 16.1 & 16.7 & 17.0 & 16.8 & 16.7 \\
\hline B6. One-time 30 percent nominal depreciation relative to the baseline in 20105 / & 15.3 & 22.5 & 22.7 & 23.7 & 24.7 & 25.3 & 25.7 & 26.6 \\
\hline
\end{tabular}

PV of debt-to-exports ratio

Baseline

A. Alternative Scenarios

A1. Key variables at their historical averages in 2009-2029 1/

A2. New public sector loans on less favorable terms in 2009-2029 2/

A3. Total new SDR allocation is used over 2011-15 in an equal annual amount

\section{B. Bound Tests}

B1. Real GDP growth at historical average minus one standard deviation in 2010-2011

B2. Export value growth at historical average minus one standard deviation in 2010-2011 3

B3. US dollar GDP deflator at historical average minus one standard deviation in 2010-2011

B4. Net non-debt creating flows at historical average minus one standard deviation in 2010-2011 4/

B5. Combination of B1-B4 using one-half standard deviation shocks

B6. One-time 30 percent nominal depreciation relative to the baseline in 20105 /
97.6

89.0

97.6

97.6

97.6

62.9

93.3

89.0
0.0

$97.6 \quad 87.1$

$97.6 \quad 121.7$

$97.6 \quad 87.1$

$97.6 \quad 89.6$

$97.6 \quad 103.6$

$97.6 \quad 87.1$
84.9

88.4

42.5

$\begin{array}{rr}93.9 & 101.6\end{array}$

$90.8 \quad 99.2$

$\begin{array}{ll}-5.9 & -10.8\end{array}$
82.7

170.4

82.7

87.1

122.9

82.7

PV of debt-to-revenue ratio

Baseline

A. Alternative Scenarios

A1. Key variables at their historical averages in 2009-2029 1/

A2. New public sector loans on less favorable terms in 2009-2029 2/

A3. Total new SDR allocation is used over 2011-15 in an equal annual amount

\section{B. Bound Tests}

B1. Real GDP growth at historical average minus one standard deviation in 2010-2011

B2. Export value growth at historical average minus one standard deviation in 2010-2011 3/

B3. US dollar GDP deflator at historical average minus one standard deviation in 2010-2011

B4. Net non-debt creating flows at historical average minus one standard deviation in 2010-2011 4/

B5. Combination of B1-B4 using one-half standard deviation shocks

B6. One-time 30 percent nominal depreciation relative to the baseline in 20105 /
$134.2 \quad 137.1$

124.7

127.6

130

129.4

121.1

112.6

$134.2 \quad 96.8$

$134.2 \quad 143.6$

$134.2 \quad 137.1$

62.4
137.9

133.4

52.1

146.8

$\begin{array}{ll}43.4 & 34.0\end{array}$

$154.6 \quad 158.0$

$151.5 \quad 155.7$

14.0

$166.5 \quad 174.2$

$143.1 \quad 123.3$ 
Table 2 b.Sierra Leone: Sensitivity Analysis for Key Indicators of Public and Publicly Guaranteed External Debt, $2009-2029$ (continued)

(In percent)

\section{Debt service-to-exports ratio}

Baseline

A. Alternative Scenarios

A1. Key variables at their historical averages in 2009-2029 1/

A2. New public sector loans on less favorable terms in 2009-2029 2/

A3. Total new SDR allocation is used over 2011-15 in an equal annual amount

\section{B. Bound Tests}

B1. Real GDP growth at historical average minus one standard deviation in 2010-201

B2. Export value growth at historical average minus one standard deviation in 2010-2011 3

B3. US dollar GDP deflator at historical average minus one standard deviation in 2010-2011

B4. Net non-debt creating flows at historical average minus one standard deviation in 2010-2011 4/

B5. Combination of B1-B4 using one-half standard deviation shocks

B6. One-time 30 percent nominal depreciation relative to the baseline in $20105 /$
4.6

4.7

$3.6 \quad 3.3$

$4.6 \quad 3.6$

3.6
4.7

$\begin{array}{llllll}3.6 & 2.7 & 2.5 & 2.4 & 0.9 & 0.3 \\ 4.6 & 4.3 & 4.6 & 5.2 & 5.0 & 5.6 \\ 5.4 & 4.9 & 4.7 & 5.2 & 5.3 & 3.7\end{array}$

$\begin{array}{llllllll} & & & & & & & \\ 4.6 & 3.6 & 4.4 & 3.7 & 3.8 & 4.1 & 4.2 & 3.3 \\ 4.6 & 4.5 & 6.8 & 6.1 & 6.2 & 6.6 & 8.0 & 5.7 \\ 4.6 & 3.6 & 4.4 & 3.7 & 3.8 & 4.1 & 4.2 & 3.3 \\ 4.6 & 3.6 & 4.4 & 3.8 & 3.8 & 4.1 & 4.3 & 3.4 \\ 4.6 & 4.2 & 5.8 & 5.1 & 5.2 & 5.5 & 6.0 & 4.6 \\ 4.6 & 3.6 & 4.4 & 3.7 & 3.8 & 4.1 & 4.2 & 3.3\end{array}$

\section{Debt service-to-revenue ratio}

Baseline

6.3

7.2

8.0

6.7

6.2

6.6

7.4

A. Alternative Scenarios

A1. Key variables at their historical averages in 2009-2029 1/

A2. New public sector loans on less favorable terms in 2009-2029 2/

A3. Total new SDR allocation is used over 2011-15 in an equal annual amount

$6.3 \quad 5.0$

$6.3 \quad 5.6$

$6.3 \quad 7.2$

$\begin{array}{rrrrrr}5.2 & 3.9 & 3.6 & 3.4 & 1.4 & 0.6 \\ 6.7 & 6.1 & 6.6 & 7.3 & 7.9 & 10.6 \\ 8.0 & 7.0 & 6.8 & 7.4 & 8.3 & 7.0\end{array}$

B. Bound Tests

B1. Real GDP growth at historical average minus one standard deviation in 2010-201

B2. Export value growth at historical average minus one standard deviation in 2010-2011 3 /

B3. US dollar GDP deflator at historical average minus one standard deviation in 2010-2011

B4. Net non-debt creating flows at historical average minus one standard deviation in 2010-2011 4/

B5. Combination of B1-B4 using one-half standard deviation shocks

B6. One-time 30 percent nominal depreciation relative to the baseline in 20105 /

$\begin{array}{llllllll}6.3 & 5.5 & 6.3 & 5.3 & 5.3 & 5.6 & 6.4 & 6.1 \\ 6.3 & 5.6 & 6.6 & 5.9 & 5.9 & 6.2 & 8.4 & 7.1 \\ 6.3 & 5.3 & 6.0 & 5.0 & 5.1 & 5.3 & 6.1 & 5.8 \\ 6.3 & 5.6 & 6.5 & 5.5 & 5.5 & 5.8 & 6.8 & 6.3 \\ 6.3 & 5.1 & 5.7 & 4.9 & 4.9 & 5.2 & 6.3 & 5.7 \\ 6.3 & 8.1 & 9.3 & 7.8 & 7.8 & 8.3 & 9.5 & 9.0 \\ & & & & & & & \\ 9.1 & 39.1 & 39.1 & 39.1 & 39.1 & 39.1 & 39.1 & 39.1\end{array}$

Grant element assumed on residual financing (i.e., financing required above baseline) 6

39.1

1/ Variables include real GDP growth, growth of GDP deflator (in U.S. dollar terms), non-interest current account in percent of GDP, and non-debt creating flows.

$2 /$ Assumes that the interest rate on new borrowing is by 2 percentage points higher than in the baseline., while grace and maturity periods are the same as in the baseline

3/ Exports values are assumed to remain permanently at the lower level, but the current account as a share of GDP is assumed to return to its baseline level after the shock (implicitly assuming

an offsetting adjustment in import levels).

4/ Includes official and private transfers and FDI.

5/ Depreciation is defined as percentage decline in dollar/local currency rate, such that it never exceeds 100 percent.

6/ Applies to all stress scenarios except for A2 (less favorable financing) in which the terms on all new financing are as specified in footnote 2. 
Figure 1. Sierra Leone: Indicators of Public and Publicly Guaranteed External Debt under Alternatives Scenarios, 2009-2029 1/
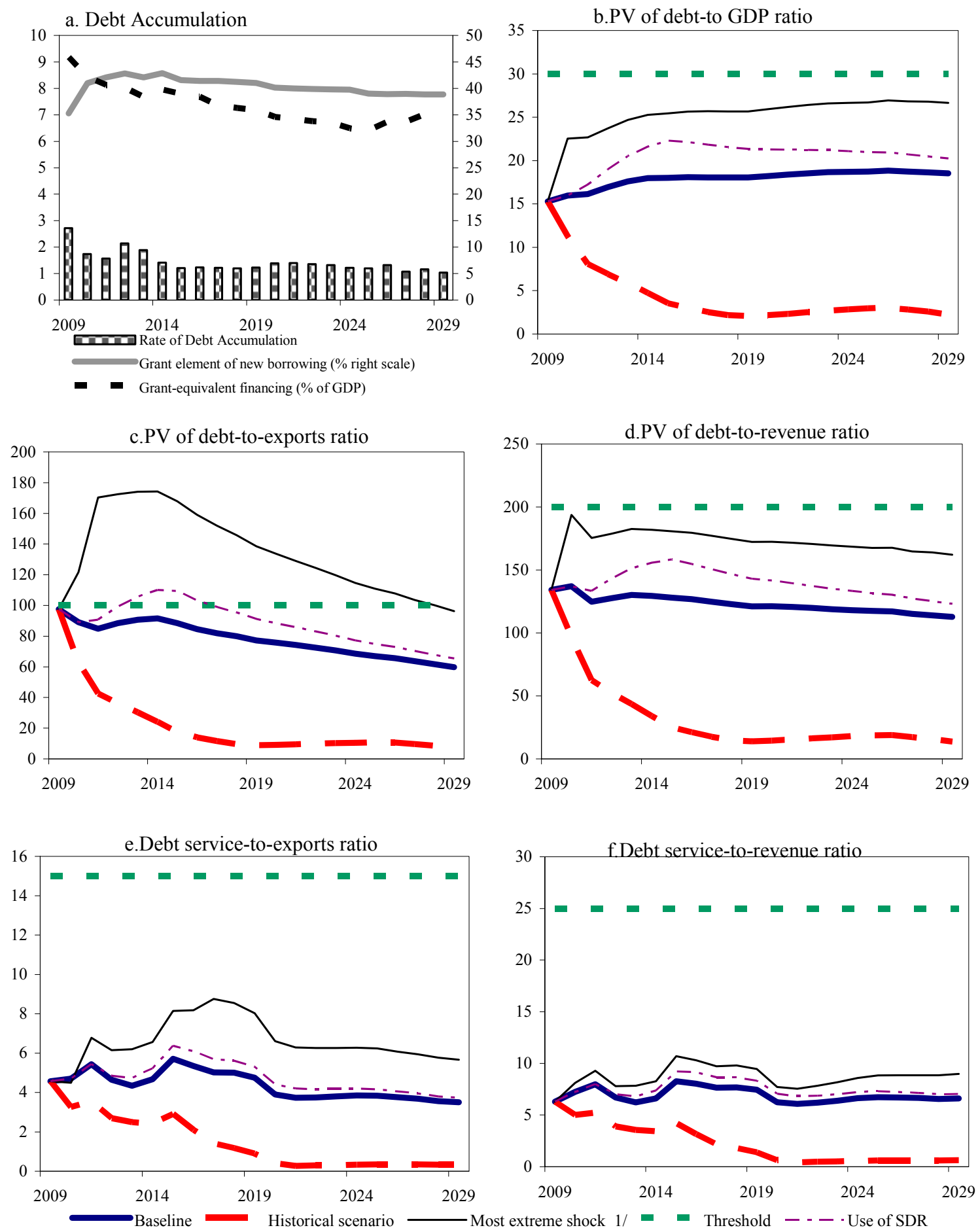

Sources: Country authorities; and staff estimates and projections.

1/ The most extreme stress test is the test that yields the highest ratio in 2019. In figure $b$. it corresponds to a One-time depreciation shock; in c. to a Exports shock; in d. to a One-time depreciation shock; in e. to a Exports shock and in figure f. to a One-time depreciation shock 
Table 3. a. Sierra Leone: Public Sector Debt Sustainability Framework, Baseline Scenario, 2006-2029

(In percent of GDP, unless otherwise indicated)

\begin{tabular}{|c|c|c|c|c|c|c|c|c|c|c|c|c|c|c|c|}
\hline & \multicolumn{3}{|c|}{ Actual } & \multirow[b]{2}{*}{ Average } & \multirow[b]{2}{*}{$\begin{array}{c}\text { Standard } \\
\text { Deviation }\end{array}$} & \multicolumn{5}{|l|}{ Estimate } & \multicolumn{5}{|c|}{ Projections } \\
\hline & 2006 & 2007 & 2008 & & & 2009 & 2010 & 2011 & 2012 & 2013 & 2014 & $\begin{array}{l}2009-14 \\
\text { Average }\end{array}$ & 2019 & 2029 & $\begin{array}{l}2015-29 \\
\text { Average }\end{array}$ \\
\hline $\begin{array}{l}\text { Public sector debt } 1 / \\
\text { o/w foreign-currency denominated }\end{array}$ & $\begin{array}{l}136.8 \\
110.4\end{array}$ & $\begin{array}{l}55.2 \\
31.7\end{array}$ & $\begin{array}{l}53.7 \\
32.9\end{array}$ & & & $\begin{array}{l}57.8 \\
39.3\end{array}$ & $\begin{array}{l}56.3 \\
39.4\end{array}$ & $\begin{array}{l}42.3 \\
28.0\end{array}$ & $\begin{array}{l}41.0 \\
28.8\end{array}$ & $\begin{array}{l}40.4 \\
29.5\end{array}$ & $\begin{array}{l}39.9 \\
29.8\end{array}$ & & $\begin{array}{l}38.8 \\
28.9\end{array}$ & $\begin{array}{l}35.5 \\
27.3\end{array}$ & \\
\hline Change in public sector debt & -37.5 & -81.7 & -1.5 & & & 4.1 & -1.5 & -13.9 & -1.3 & -0.6 & -0.5 & & -0.7 & -0.2 & \\
\hline Identified debt-creating flows & -22.3 & -20.4 & -3.2 & & & 3.8 & -0.5 & -1.1 & -0.3 & -0.1 & -0.1 & & 0.0 & 0.9 & \\
\hline Primary deficit & -1.2 & -1.1 & 2.7 & -0.7 & 1.9 & 2.7 & 2.3 & 2.4 & 1.9 & 1.7 & 1.3 & 2.1 & 1.3 & 1.9 & 1.6 \\
\hline Revenue and grants & 20.1 & 15.7 & 16.0 & & & 18.7 & 18.6 & 19.5 & 19.7 & 19.9 & 20.5 & & 21.1 & 22.2 & \\
\hline of which: grants & 8.2 & 4.8 & 4.5 & & & 7.3 & 7.0 & 6.6 & 6.4 & 6.3 & 6.6 & & 6.1 & 5.7 & \\
\hline Primary (noninterest) expenditure & 18.9 & 14.6 & 18.6 & & & 21.4 & 20.9 & 21.9 & 21.6 & 21.6 & 21.8 & & 22.4 & 24.1 & \\
\hline Automatic debt dynamics & -20.1 & -18.5 & -5.6 & & & 1.4 & -2.7 & -3.3 & -2.1 & -1.7 & -1.3 & & -1.2 & -1.0 & \\
\hline Contribution from interest rate/growth differential & -18.7 & -17.9 & -6.2 & & & -0.1 & -0.8 & -3.3 & -2.3 & -1.7 & -1.3 & & -1.2 & -1.0 & \\
\hline of which: contribution from average real interest rate & -6.9 & -9.6 & -3.3 & & & 2.0 & 1.7 & -0.4 & 0.1 & 0.8 & 0.8 & & 0.7 & 0.7 & \\
\hline of which: contribution from real GDP growth & -11.8 & -8.3 & -2.9 & & & -2.1 & -2.6 & -2.9 & -2.4 & -2.5 & -2.1 & & -1.9 & -1.7 & \\
\hline Contribution from real exchange rate depreciation & -1.4 & -0.6 & 0.6 & & & 1.5 & -1.9 & 0.0 & 0.1 & 0.0 & 0.0 & & & & \\
\hline Other identified debt-creating flows & -1.1 & -0.8 & -0.3 & & & -0.4 & -0.2 & -0.2 & -0.2 & -0.1 & -0.1 & & -0.1 & 0.0 & \\
\hline Privatization receipts (negative) & -0.1 & -0.1 & -0.1 & & & -0.2 & 0.0 & 0.0 & 0.0 & 0.0 & 0.0 & & 0.0 & 0.0 & \\
\hline Recognition of implicit or contingent liabilities & 0.0 & 0.0 & 0.0 & & & 0.0 & 0.0 & 0.0 & 0.0 & 0.0 & 0.0 & & 0.0 & 0.0 & \\
\hline Debt relief (HIPC and other) & -1.0 & -0.7 & -0.2 & & & -0.2 & -0.2 & -0.2 & -0.2 & -0.1 & -0.1 & & -0.1 & 0.0 & \\
\hline Other (specify, e.g. bank recapitalization) & 0.0 & 0.0 & 0.0 & & & 0.0 & 0.0 & 0.0 & 0.0 & 0.0 & 0.0 & & 0.0 & 0.0 & \\
\hline Residual, including asset changes & -15.1 & -61.2 & 1.7 & & & 0.3 & -1.0 & -12.9 & -0.9 & -0.5 & -0.4 & & -0.7 & -1.1 & \\
\hline \multicolumn{16}{|l|}{ Other Sustainability Indicators } \\
\hline PV of public sector debt & 26.4 & 23.5 & 33.7 & & & 34.6 & 33.3 & 30.7 & 29.2 & 28.5 & 28.1 & & 27.9 & 26.7 & \\
\hline $\mathrm{o} / \mathrm{w}$ foreign-currency denominated & 0.0 & 0.0 & 12.9 & & & 16.1 & 16.5 & 16.4 & 17.0 & 17.6 & 18.0 & & 18.0 & 18.5 & \\
\hline $\mathrm{o} / \mathrm{w}$ external & $\ldots$ & $\ldots$ & 11.8 & & & 15.3 & 16.0 & 16.1 & 16.9 & 17.6 & 18.0 & & 18.0 & 18.5 & \\
\hline $\mathrm{PV}$ of contingent liabilities (not included in public sector debt) & $\ldots$ & $\ldots$ & & & & $\ldots$ & $\ldots$ & & & & $\ldots$ & & $\ldots$ & $\ldots$ & \\
\hline Gross financing need $2 /$ & 5.5 & 2.4 & 5.1 & & & 4.8 & 4.8 & 4.8 & 4.0 & 3.6 & 3.1 & & 3.4 & 3.7 & \\
\hline $\mathrm{PV}$ of public sector debt-to-revenue and grants ratio (in percent) & 131.5 & 149.2 & 211.1 & & & 184.7 & 179.2 & 157.6 & 148.3 & 143.6 & 136.9 & & 132.6 & 120.4 & \\
\hline PV of public sector debt-to-revenue ratio (in percent) & 222.9 & 215.0 & 294.9 & & & 303.5 & 286.4 & 237.7 & 220.4 & 210.6 & 202.3 & & 187.2 & 162.4 & \\
\hline o/w external 3/ & & & 102.8 & & & 134.2 & 137.2 & 124.8 & 127.7 & 130.1 & 129.5 & & 120.7 & 112.2 & \\
\hline Debt service-to-revenue and grants ratio (in percent) $4 /$ & 33.3 & 22.6 & 15.1 & & & 11.3 & 13.2 & 12.2 & 10.3 & 9.4 & 9.1 & & 9.8 & 8.2 & \\
\hline Debt service-to-revenue ratio (in percent) $4 /$ & 56.4 & 32.6 & 21.1 & & & 18.6 & 21.1 & 18.5 & 15.4 & 13.8 & 13.4 & & 13.8 & 11.1 & \\
\hline Primary deficit that stabilizes the debt-to-GDP ratio & 36.3 & 80.5 & 4.2 & & & -1.4 & 3.9 & 16.4 & 3.2 & 2.3 & 1.8 & & 2.0 & 2.1 & \\
\hline \multicolumn{16}{|l|}{ Key macroeconomic and fiscal assumptions } \\
\hline Real GDP growth (in percent) & 7.3 & 6.4 & 5.5 & 6.8 & 0.8 & 4.0 & 4.7 & 5.5 & 6.0 & 6.5 & 5.5 & 5.4 & 5.0 & 5.0 & 5.0 \\
\hline Average nominal interest rate on forex debt (in percent) & 1.6 & 0.4 & 1.3 & 1.3 & 0.5 & 0.8 & 1.1 & 0.9 & 0.9 & 0.9 & 0.9 & 0.9 & 1.0 & 1.0 & 1.0 \\
\hline Average real interest rate on domestic debt (in percent) & -0.7 & -2.4 & -2.7 & -3.4 & 3.5 & 0.8 & 2.4 & 3.0 & 4.0 & 5.8 & 6.5 & 3.8 & 8.6 & 10.3 & 9.2 \\
\hline Real exchange rate depreciation (in percent, + indicates depreciation) & -1.1 & -0.6 & 2.3 & -1.3 & 2.5 & 4.5 & ... & & & & $\ldots$ & $\ldots$ & & & \\
\hline Inflation rate (GDP deflator, in percent) & 9.6 & 10.8 & 11.2 & 12.6 & 3.5 & 6.3 & 7.5 & 6.3 & 5.5 & 4.4 & 4.2 & 5.7 & 3.3 & 2.2 & 3.0 \\
\hline Growth of real primary spending (deflated by GDP deflator, in percent) & 0.0 & -0.2 & 0.3 & 0.0 & 0.2 & 0.2 & 0.0 & 0.1 & 0.0 & 0.1 & 0.1 & 0.1 & 0.1 & 0.1 & 0.1 \\
\hline Grant element of new external borrowing (in percent) & $\ldots$ & $\ldots$ & & $\ldots$ & & 35.3 & 41.0 & 42.1 & 42.8 & 42.1 & 42.8 & 41.0 & 41.0 & 38.8 & ... \\
\hline
\end{tabular}

Sources: Country authorities; and

[Indicate coverage of public sector, e.g., general government or nonfinancial public sector. Also whether net or gross debt is used.]

2/ Gross financing need is defined as the primary deficit plus debt service plus the stock of short-term debt at the end of the last period

$5 /$ Historical averages and standard deviations are derived over the past 5 years. A longer period is not considered due to large fluctuations in the post-conflict economic data. 
Table 3.b.Sierra Leone: Sensitivity Analysis for Key Indicators of Public Debt 2009-2029

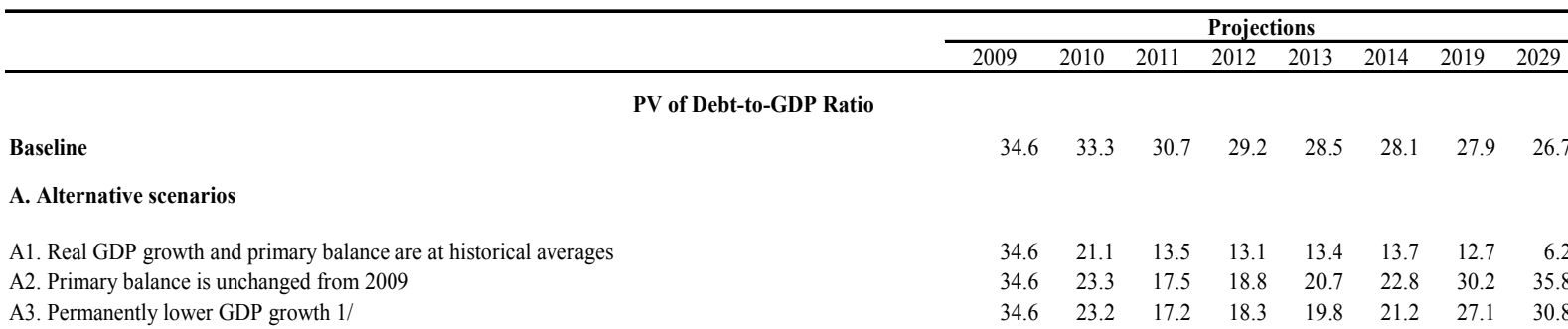

\section{B. Bound tests}

B1. Real GDP growth is at historical average minus one standard deviations in 2010-2011

B2. Primary balance is at historical average minus one standard deviations in 2010-2011

B3. Combination of B1-B2 using one half standard deviation shocks

B4. One-time 30 percent real depreciation in 2010

B5. 10 percent of GDP increase in other debt-creating flows in 2010

PV of Debt-to-Revenue Ratio 2/

$\begin{array}{llllllll}34.6 & 22.8 & 16.8 & 17.6 & 18.9 & 20.2 & 24.8 & 25.4 \\ 34.6 & 22.5 & 15.9 & 16.9 & 18.4 & 19.8 & 25.1 & 26.7 \\ 34.6 & 21.7 & 14.6 & 15.5 & 16.8 & 18.1 & 22.6 & 23.3 \\ 34.6 & 26.0 & 24.4 & 24.5 & 25.4 & 26.4 & 29.7 & 29.0 \\ 34.6 & 28.5 & 22.2 & 22.9 & 24.2 & 25.4 & 29.9 & 29.7\end{array}$

Baseline

$\begin{array}{lllllll}179.8 & 179.2 & 157.6 & 148.3 & 143.6 & 136.9 & 132.6\end{array}$

120.4

\section{A. Alternative scenarios}

A1. Real GDP growth and primary balance are at historical averages

A2. Primary balance is unchanged from 2009

A3. Permanently lower GDP growth $1 /$

$\begin{array}{rrrrrrrr}184.7 & 114.1 & 70.0 & 67.4 & 68.1 & 67.5 & 62.3 & 29.9 \\ 184.7 & 125.2 & 89.6 & 95.5 & 104.4 & 111.3 & 143.6 & 161.3 \\ 184.7 & 124.4 & 88.3 & 92.5 & 99.3 & 103.2 & 128.0 & 137.6\end{array}$

\section{B. Bound tests}

B1. Real GDP growth is at historical average minus one standard deviations in 2010-2011

B2. Primary balance is at historical average minus one standard deviations in 2010-2011

B3. Combination of B1-B2 using one half standard deviation shocks

B4. One-time 30 percent real depreciation in 2010

B5. 10 percent of GDP increase in other debt-creating flows in 2010

$\begin{array}{rrrrrrrr}184.7 & 123.0 & 86.3 & 89.7 & 95.6 & 98.7 & 118.1 & 114.8 \\ 184.7 & 121.0 & 81.7 & 86.0 & 92.7 & 96.5 & 119.0 & 120.7 \\ 184.7 & 117.2 & 75.5 & 79.2 & 85.3 & 88.6 & 108.2 & 105.9 \\ 184.7 & 139.9 & 125.2 & 124.5 & 127.8 & 128.8 & 141.3 & 130.8 \\ 184.7 & 153.0 & 114.0 & 116.3 & 121.8 & 124.0 & 141.9 & 134.2\end{array}$

Debt Service-to-Revenue Ratio 2/

Baseline

$\begin{array}{llllllll}11.0 & 13.2 & 12.2 & 10.3 & 9.4 & 9.1 & 9.8 & 8.2\end{array}$

\section{A. Alternative scenarios}

A1. Real GDP growth and primary balance are at historical averages

A2. Primary balance is unchanged from 2009

A3. Permanently lower GDP growth $1 /$

$\begin{array}{rrrrrrrr}11.0 & 13.9 & 13.8 & 12.1 & 11.1 & 10.2 & 10.0 & 7.2 \\ 11.0 & 14.1 & 14.2 & 12.9 & 12.0 & 11.2 & 12.2 & 12.8 \\ 11.0 & 14.1 & 14.2 & 12.9 & 12.0 & 11.2 & 12.0 & 12.2\end{array}$

\section{B. Bound tests}

B1. Real GDP growth is at historical average minus one standard deviations in 2010-2011

B2. Primary balance is at historical average minus one standard deviations in 2010-2011

B3. Combination of B1-B2 using one half standard deviation shocks

B4. One-time 30 percent real depreciation in 2010

B5. 10 percent of GDP increase in other debt-creating flows in 2010

$\begin{array}{llllllll}11.0 & 14.0 & 14.1 & 12.7 & 11.8 & 11.0 & 11.7 & 11.3 \\ 11.0 & 14.1 & 14.1 & 12.7 & 11.8 & 11.0 & 11.8 & 11.5 \\ 11.0 & 14.0 & 13.9 & 12.4 & 11.4 & 10.7 & 11.5 & 10.9 \\ 11.0 & 15.3 & 17.0 & 15.3 & 14.2 & 13.5 & 14.7 & 14.9 \\ 11.0 & 14.1 & 14.7 & 13.7 & 12.3 & 11.5 & 12.1 & 12.3\end{array}$

Sources: Country authorities; and staff estimates and projections.

1/ Assumes that real GDP growth is at baseline minus one standard deviation divided by the square root of the length of the projection period.

2 / Revenues are defined inclusive of grants. 
Figure 2.Sierra Leone: Indicators of Public Debt Under Alternative Scenarios, 2009-2029 1/
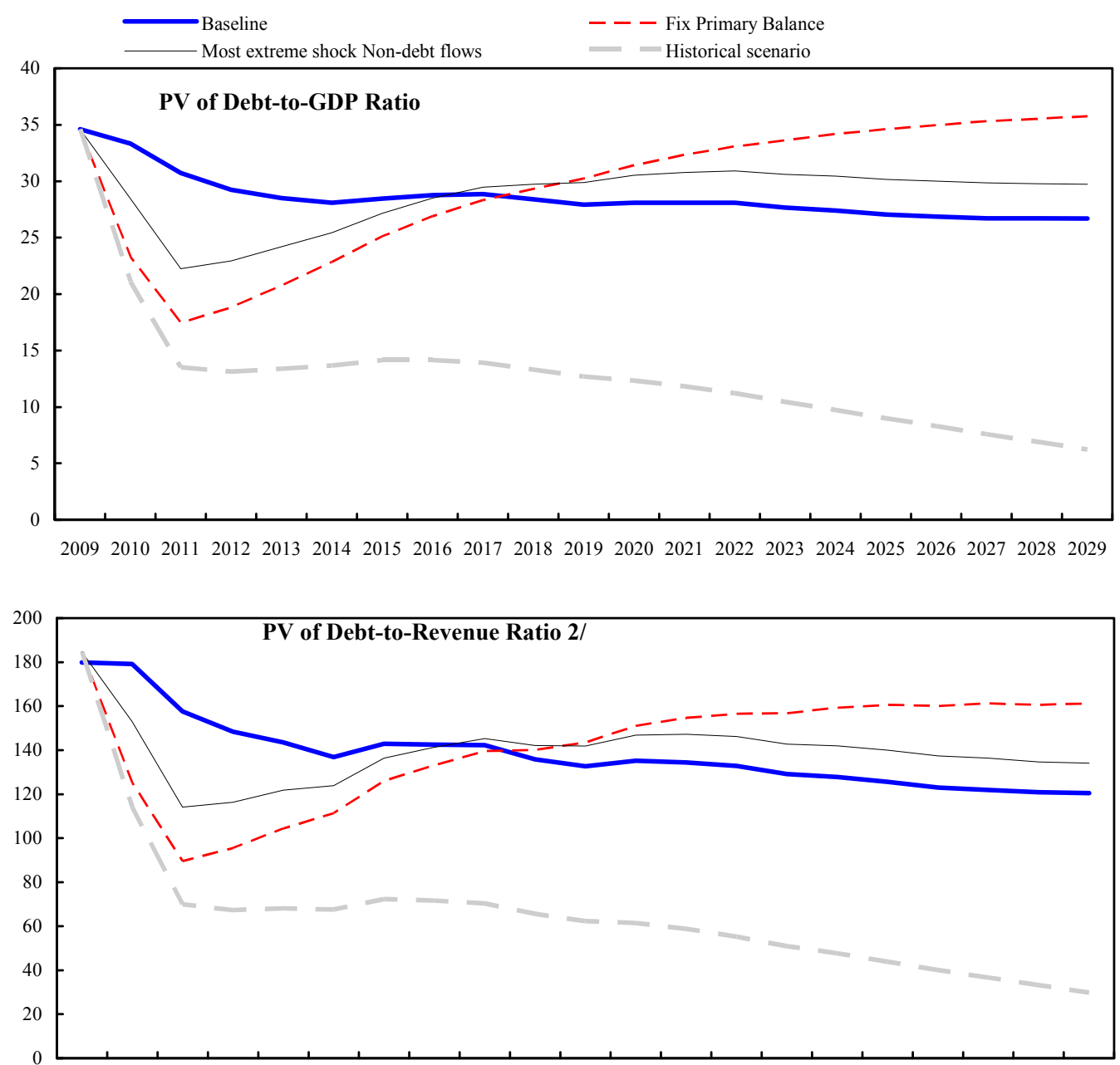

$20092010201120122013201420152016201720182019202020212022 \quad 2023202420252026202720282029$

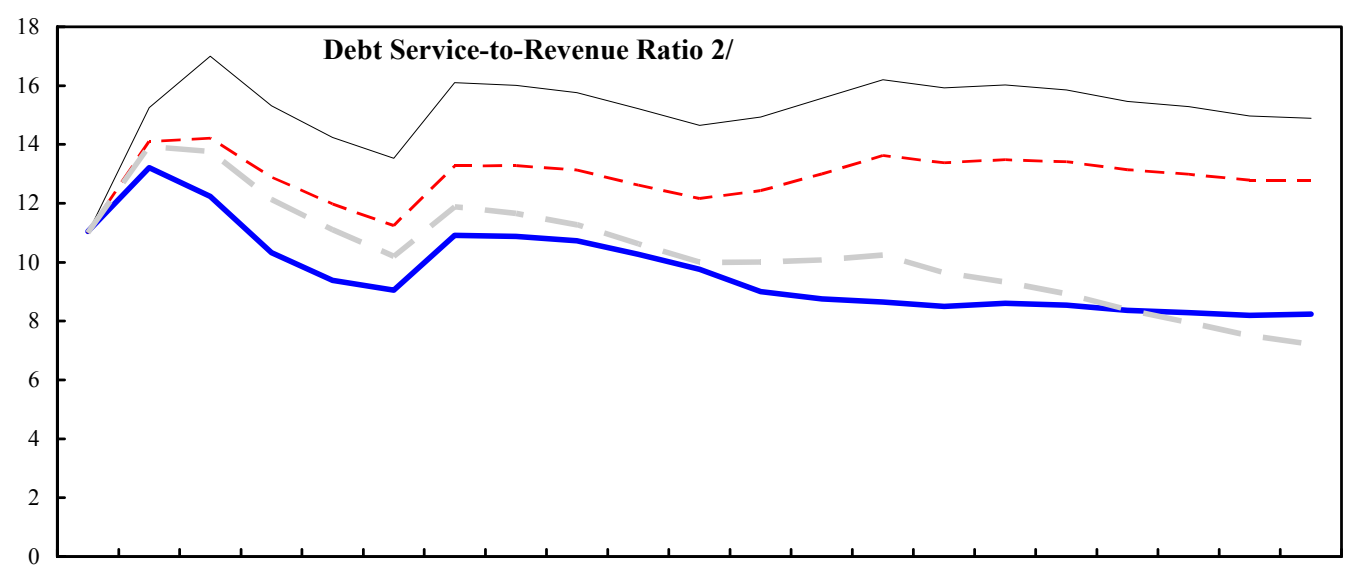

200920102011201220132014201520162017201820192020202120222023202420252026202720282029

Sources: Country authorities; and staff estimates and projections.

$1 /$ The most extreme stress test is the test that yields the highest ratio in 2019.

$2 /$ Revenues are defined inclusive of grants. 


\section{INTERNATIONAL MONETARY FUND}

SIERRA LEONE

Fifth Review Under the Arrangement Under the Poverty Reduction and Growth Facility, Request for Waiver of Nonobservance of Performance Criterion, Request for Modification of Performance Criteria, and Financing Assurances Review-Informational Annex

Prepared by the African Department (In collaboration with other departments)

December 7, 2009

Contents

I. Relations with the Fund

II. Joint World Bank-IMF Work Program, 2009-10 ........................................................

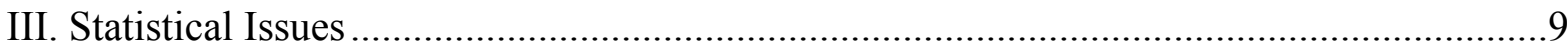




\section{Appendix I. Relations with the Fund}

(As of September 30, 2009)

I. Membership Status: Joined 9/10/62; Article VIII

II. General Resources Account:

Quota

Fund holdings of currency

Reserve position

III. SDR Department:

Net cumulative allocation

Holdings

IV. Outstanding Purchases and Loans:

PRGF Arrangements
SDR Million

103.70

103.69

0.02

SDR Million

99.51

121.04

SDR Million

46.70

Amount

Approved

(SDR Million)

51.88

130.84

101.90
$\%$ Quota

100.00

99.99

0.02

\% Allocation

100.00

121.65

$\%$ Quota

45.03

V. Latest Financial Arrangements:

\begin{tabular}{|c|c|c|c|c|}
\hline Type & Date & Date & (SDR Million) & (SDR Million) \\
\hline PRGF & $5 / 10 / 06$ & $5 / 9 / 10$ & 51.88 & 32.70 \\
\hline PRGF & $9 / 26 / 01$ & $6 / 25 / 05$ & 130.84 & 130.84 \\
\hline PRGF & $3 / 28 / 94$ & $5 / 04 / 98$ & 101.90 & 96.85 \\
\hline
\end{tabular}

\section{Projected Payments to Fund ${ }^{1}$}

(SDR million; based on current use of resources and present holdings of SDRs):

Principal

Charges/interest

\begin{tabular}{lllll}
\multicolumn{5}{c}{ Forthcoming } \\
\hline$\underline{2009}$ & $\frac{2010}{1.40}$ & $\frac{2011}{3.27}$ & $\frac{2012}{4.62}$ & $\frac{2013}{4.62}$ \\
$\underline{0.12}$ & $\underline{0.24}$ & $\underline{0.23}$ & $\underline{0.21}$ & $\underline{0.19}$ \\
$\underline{0.12}$ & 1.64 & $\underline{3.50}$ & $\underline{4.83}$ & $\underline{4.81}$
\end{tabular}

VII. Implementation of HIPC Initiative

Commitment of HIPC Initiative assistance

\section{Enhanced} Framework

Decision point date

March 2002

Assistance committed (NPV terms)

By all creditors (US\$ million) ${ }^{2}$

\footnotetext{
${ }^{1}$ When a member has overdue financial obligations outstanding for more than three months, the amount will be be shown in this section.

${ }^{2}$ Assistance committed under the original framework is expressed in net present value (NPV) terms at the
} 
Of which: IMF assistance (US\$ million)

125.21

(SDR equivalent in millions)

100.00

Completion point date

December 2006

Disbursement of IMF assistance (SDR million)

Amount disbursed

100.00

Interim assistance

Completion point balance

Additional disbursement of interest income ${ }^{3}$

6.58

Total disbursements

106.58

VIII. Implementation of Multilateral Debt Relief Initiative (MDRI):

I. MDRI-eligible debt (SDR Million) ${ }^{4}$

117.34

Financed by: MDRI Trust

76.75

Remaining HIPC resources

40.59

II. Debt Relief by facility (SDR Million)

\begin{tabular}{lrrr} 
& \multicolumn{3}{c}{ Eligible Debt } \\
\cline { 2 - 3 } Delivery Date & $\underline{\text { GRA }}$ & $\underline{\text { PRGF }}$ & Total \\
December 2006 & N/A & 117.34 & 117.34
\end{tabular}

\section{Safeguards Assessment}

Pursuant to IMF policy, the Bank of Sierra Leone was subject to a safeguards assessment with respect to the PRGF arrangement approved on May 10, 2006. The assessment, which was completed on June 12, 2006, proposed recommendations to address new and continuing vulnerabilities in financial reporting, internal audits, and internal controls. An update of the 2006 safeguards assessment report was completed in October 2009.

completion point, and assistance committed under the enhanced framework is expressed in NPV terms at the decision point. Hence these two amounts cannot be added.

${ }^{3}$ Under the enhanced framework, an additional disbursement is made at the completion point corresponding to interest income earned on the amount committed at the decision point but not disbursed during the interim.

4. The MDRI provides 100 percent debt relief to eligible member countries that qualified for the assistance. Grant assistance from the MDRI Trust and HIPC resources provide debt relief to cover the full stock of debt owed to the Fund as of 2004 that remains outstanding at the time the member qualifies for debt relief. 


\section{Exchange Rate Arrangement}

For customs valuation purposes and for official transactions, the Bank of Sierra Leone (BSL) calculates an official exchange rate every Friday morning as the weighted average of the auction rate, the commercial bank mid-rate, and the bureau mid-rate in the previous week. Commercial banks may buy foreign exchange from and sell it to individual customers and may trade among themselves or with the BSL on a freely negotiable basis. As of October 08, 2009, the BSL mid-rate was Le 3,645.58=US\$1.

In March 2008 the exchange rate regime was reclassified as "a conventional peg" to reflect the nominal stability of exchange rate developments under de facto management through the auction by the BSL as the only seller of foreign exchange. Effective February 2, 2009, the de facto exchange rate arrangement was reclassified from a conventional pegged arrangement to "stabilized" retroactively to April 30, 2008, due to a revision of the classification methodology.

With effect December 14, 1995, Sierra Leone has accepted the obligations of Article VIII, Sections 2, 3, and 4. On June 29, 2001, Sierra Leone removed the remaining exchange restriction, in the form of a tax clearance certificate required for payments and transfers of certain types of current international transactions. Sierra Leone continues to maintain an exchange system free of restrictions on the making of payments and transfers for current international transactions.

\section{Article IV Consultation}

The Executive Board concluded the 2008 Article IV consultation on December 22, 2008. The next Article IV consultation will be held in accordance with the decision on consultation cycles approved on July 15, 2002.

\section{Technical Assistance}

\begin{tabular}{|l|l|l|}
\hline Department & Purpose & Date \\
\hline FAD & Evaluation of progress in PFM reforms & March 2009 \\
\hline & $\begin{array}{l}\text { Assist in designing a simplified regime for } \\
\text { small taxpayers }\end{array}$ & January 2009 \\
\hline & $\begin{array}{l}\text { Evaluation of progress on tax } \\
\text { administration reform and VAT } \\
\text { preparation }\end{array}$ & September 2008 \\
\hline & $\begin{array}{l}\text { Evaluation of progress on PFM reforms } \\
\text { Implementation of public financial }\end{array}$ & July 2008 \\
\hline management reforms & March 2008 \\
\hline
\end{tabular}




\begin{tabular}{|c|c|c|}
\hline Department & Purpose & Date \\
\hline & $\begin{array}{l}\text { Tax administration reforms for successful } \\
\text { VAT implementation }\end{array}$ & February 2008 \\
\hline & $\begin{array}{l}\text { Evaluation of progress of revenue } \\
\text { administration modernization }\end{array}$ & November 2007 \\
\hline & $\begin{array}{l}\text { Assist the authorities in developing a } \\
\text { comprehensive revenue administration } \\
\text { reform strategy }\end{array}$ & February 2006 \\
\hline & $\begin{array}{l}\text { Review the system of fiscal incentives, } \\
\text { especially the special tax regimes applied } \\
\text { to the mining and petroleum sectors }\end{array}$ & March/April 2004 \\
\hline & $\begin{array}{l}\text { Review the tariff and indirect tax system, } \\
\text { National Revenue Authority, and tax and } \\
\text { customs administration }\end{array}$ & February 2004 \\
\hline LEG & $\begin{array}{l}\text { TA on a legal review of the Bank of Sierra } \\
\text { Leone Act of } 2000\end{array}$ & March/April 2007 \\
\hline \multirow[t]{10}{*}{$\mathrm{MCM}$} & Follow-up assessment on TA needs & June 2009 \\
\hline & $\begin{array}{l}\text { Follow-up assessment of banking } \\
\text { supervision processes at BSL }\end{array}$ & March 2009 \\
\hline & $\begin{array}{l}\text { Follow-up assessment on progress in } \\
\text { strengthening central bank internal } \\
\text { auditing }\end{array}$ & March 2009 \\
\hline & $\begin{array}{l}\text { BSL: Internal audit and management of } \\
\text { the central bank }\end{array}$ & October 2008 \\
\hline & $\begin{array}{l}\text { Monetary policy, banking supervision, } \\
\text { and recapitalization of the BSL }\end{array}$ & April/May 2008 \\
\hline & Recapitalization of the BSL & February 2008 \\
\hline & $\begin{array}{l}\text { Assist the } \mathrm{BSL} \text { in bringing its accounting } \\
\text { practices up to international best practice }\end{array}$ & $\begin{array}{l}\text { November/December } \\
2007\end{array}$ \\
\hline & $\begin{array}{l}\text { Foreign exchange, monetary operations, } \\
\text { and central bank recapitalization }\end{array}$ & November 2007 \\
\hline & $\begin{array}{l}\text { Assist the BSL in setting up an action plan } \\
\text { for implementing FSAP } \\
\text { recommendations/financial sector reforms }\end{array}$ & June 2007 \\
\hline & $\begin{array}{l}\text { Foreign exchange, monetary operations, } \\
\text { and central bank recapitalization }\end{array}$ & January/February 2007 \\
\hline
\end{tabular}




\begin{tabular}{|l|l|l|}
\hline Department & Purpose & Date \\
\hline MFD & Multitopic technical assistance & November 2005 \\
\hline STA & $\begin{array}{l}\text { Multitopic technical assistance } \\
\text { Assist the authorities in implementing the } \\
\text { international standards recommended in } \\
\text { the } \text { Monetary and Financial Statistics } \\
\text { Manual }\end{array}$ & $\begin{array}{l}\text { November 2004 } \\
\text { April 2009 }\end{array}$ \\
\hline & $\begin{array}{l}\text { GDDS mission on national accounts } \\
\text { statistics }\end{array}$ & March 2009 \\
\hline & $\begin{array}{l}\text { GDDS mission on national accounts } \\
\text { statistics }\end{array}$ & September/October 2008 \\
\hline & $\begin{array}{l}\text { GDDS mission to review the } \\
\text { methodological basis for compiling } \\
\text { national accounts aggregates }\end{array}$ & January 2008 \\
\hline & $\begin{array}{l}\text { GDDS project on implementation of a } \\
\text { Standardized Report Form (SRF) }\end{array}$ & June 2007 \\
\hline & $\begin{array}{l}\text { GDDS project on reconciling monetary } \\
\text { and fiscal accounts }\end{array}$ & August 2006 \\
\hline & GDDS project on national accounts & January 2006 \\
\hline & GDDS project on national accounts & February 2005 \\
\hline & $\begin{array}{l}\text { GDDS project on reconciling monetary } \\
\text { and fiscal accounts }\end{array}$ & February 2005 \\
\hline & $\begin{array}{l}\text { GDDS project on reconciling monetary } \\
\text { and fiscal accounts. }\end{array}$ & October 2004 \\
& $\begin{array}{l}\text { GDDS project on balance of payments } \\
\text { issues }\end{array}$ & September/October 2004 \\
\hline & $\begin{array}{l}\text { GDDS project on national accounts } \\
\text { planning }\end{array}$ & September 2004 \\
\hline & GDDS project on national accounts & April 2004 \\
\hline & GDDS project on strategic planning & March 2004 \\
\hline & august 2004 \\
\hline & & \\
\hline & &
\end{tabular}

\section{Resident Representative}

Mr. Meshack Tjirongo will assume responsibility for the Fund office in November 2009 as a new resident representative. Mr. Tjirongo will cover both The Gambia and Sierra Leone and will be stationed in Freetown. 
Appendix II. Sierra Leone Joint World Bank-IMF Work Program, 2009-10

\begin{tabular}{|c|c|c|c|}
\hline Title & Activities & $\begin{array}{l}\text { Provisional Timing } \\
\text { of Mission }\end{array}$ & $\begin{array}{l}\text { Expected Delivery } \\
\text { Date }\end{array}$ \\
\hline
\end{tabular}

\section{A. Mutual Information on Relevant Work Programs}

\begin{tabular}{|c|c|}
\hline $\begin{array}{l}\text { World Bank } \\
\text { work program }\end{array}$ & Public Expenditure Review \\
\hline & Country Assistance Strategy \\
\hline & $\begin{array}{l}\text { Third Governance Reform and Growth } \\
\text { Credit (GRGC 3) }\end{array}$ \\
\hline & Mineral Sector TA \\
\hline & Financial Sector TA \\
\hline & $\begin{array}{l}\text { First Programmatic Development Policy } \\
\text { Credit }\end{array}$ \\
\hline & IRCBP Supervision \\
\hline & IPFMRP (PFM Project) Supervision \\
\hline & Decentralized Service Delivery Spn. \\
\hline $\begin{array}{l}\text { IMF work } \\
\text { program }\end{array}$ & $\begin{array}{l}\text { Sixth PRGF review and negotiation of a } \\
\text { new program }\end{array}$ \\
\hline & $\begin{array}{l}2010 \text { Article IV consultation and first } \\
\text { PRGF review }\end{array}$ \\
\hline
\end{tabular}

\section{Technical Assistance}

FAD:

- Revenue administration and tax reform (GST implementation)

- Public financial management (streamlining budget execution)

- Framework for retail fuel price setting

MCM

- Central bank audit

- Bank supervision

- Payment systems

- Multi-topic (bank supervision, monetary
February 2009

ongoing dialogue

June 2009

May 2009

November 2009

January 2010

December 2009

Multiple 2010

Multiple 2010

February 2010

September 2010

December 2010

December 2009

December 2009

November 2009

December 2009

June 2010

September 2010

N/A

N/A

N/A

April 2010

February 2010

March 2010

November 2009

December 2009

February 2010

March 2010

October 2009

November 2009

Fourth quarter 2009

December 2009

December 2009

December 2009

March 2010

April 2010 


\begin{tabular}{|c|c|c|c|}
\hline Title & Activities & $\begin{array}{l}\text { Provisional Timing } \\
\text { of Mission }\end{array}$ & $\begin{array}{l}\text { Expected Deliver } \\
\text { Date }\end{array}$ \\
\hline & operations, audit) & & \\
\hline & STA & & \\
\hline & - Monetary and financial statistics & First quarter 2010 & First quarter 2010 \\
\hline \multicolumn{4}{|c|}{ B. Requests for Work Program Inputs } \\
\hline $\begin{array}{l}\text { Fund request to } \\
\text { Bank }\end{array}$ & Public expenditure review & February 2009 & December 2009 \\
\hline \multirow[t]{2}{*}{$\begin{array}{l}\text { Bank request to } \\
\text { Fund }\end{array}$} & Medium-term macroeconomic framework & & Continuous \\
\hline & C. Agreement on Joint Produ & and Missions & \\
\hline \multirow{3}{*}{$\begin{array}{l}\text { Joint products in } \\
\text { FY } 2010\end{array}$} & DSA & & December 2009 \\
\hline & DSA & & December 2010 \\
\hline & $\begin{array}{l}\text { Assisting the authorities with the } \\
\text { implementation of their financial sector } \\
\text { reform strategy }\end{array}$ & & Continuous \\
\hline
\end{tabular}




\section{Appendix III. Statistical Issues}

1. Data provided to the Fund are affected by serious shortcomings that significantly hamper surveillance. The prolonged civil war resulted in a virtual collapse of statistical systems in the late 1990s. Serious deficiencies still affect balance of payments statistics, national accounts, and social indicators. The authorities are cooperating fully in providing data to the Fund and regularly disseminating economic and financial data to the public. The country participates in the GDDS, but its metadata need to be updated.

2. A major and sustained improvement in the coverage and timeliness of economic data will require greater interagency coordination and restructuring the institutional framework. Statistics Sierra Leone (SSL) continues to face a shortage of budgetary resources which adversely affects compilation of national accounts and price statistics. Since March 2004, and with support from the GDDS Anglophone Africa Project, fourteen national accounts TA missions visited Freetown, the most recent in March 2009. These missions reviewed source data, methodologies, compilation, and dissemination issues and assisted with data development and improvements in methodology.

\section{National accounts}

3. The coverage of private sector activities in the national accounts is hampered by an outdated business directory and low response rates to surveys. In particular, small businesses, which represent a very high proportion of business activity, are not adequately captured in national account statistics. The consumer price index (CPI) is used extensively to derive estimates of GDP at constant prices. AFR missions continue to make their own estimates and projections of key national accounts aggregates, as well as making adjustments to historical data using new information.

4. Efforts to reconstruct the national accounts estimates commenced during 2003/04, with the main effort being to prepare new estimates for the period starting in 2001. Because resource constraints significantly the production of final estimates, only preliminary data for 2001-04 were made available. Efforts are underway to prepare revised national accounts based on a 2005 benchmark and using 2005 prices to compile constant price estimates. However, shortages of resources and staff continue to pose major constraints to the statistical development undertaken by SSL.

\section{Prices}

5. The SSL compiles the CPI monthly and publishes it with a lag of about three weeks. It has been rebased to 2003 using the 2003 Sierra Leone integrated household survey and continues to cover the capital city and three towns. While a national CPI is being compiled, the authorities continue to publish the old CPI $(1992=100)$. To avoid confusion, the authorities should be encouraged to cease publication of the old series, although they may 
wish to publish a separate report on the changes between base periods. The production of producer price indices remains a medium-term goal planned to start in 2009.

\section{Government finance statistics}

6. The budget reporting system was established with assistance from the Fund/UNDP technical assistance project. Monthly data on central government revenue, current expenditure, and financing are provided with appropriate detail. The European Commission is providing technical assistance to the Accountant General's Office to improve the timeliness and quality of fiscal data. Fiscal data are reported to AFR but annual data for publication in the GFS Yearbook are no longer being submitted (the most recent data refer to 2004). Fiscal data only cover the central government, not extra budgetary agencies and local governments.

7. There is an urgent need for more timely and accurate data on foreign-financed development projects. Reports on implementation of the development budget and its financing are currently not produced in a format that is suitable for budget analysis because the necessary data are not available. There is also need for quality control of the final data. The authorities are currently considering introduction of a flash reporting system for government expenditure in general and for foreign aid-financed projects. As part of the GDDS regional project for Anglophone African countries, work has been undertaken to reconcile fiscal and monetary data and to improve the coverage and classification of the two data sets.

\section{Monetary statistics}

8. The main components of the central bank balance sheet are available daily and weekly; this system provides an early warning system on key financial targets. The full monetary survey is compiled by the BSL with a lag of about six weeks; it has comprehensive coverage of commercial banks.

9. There has been some progress in the compilation of data in line with the 2000 Monetary and Financial Statistics Manual. The BSL, with assistance from STA, has completed the preparation of a standardized report form (SRF) for the central bank, but it has not yet started regular reporting to the IMF. Work on preparation of the SRF for other depository corporations continues.

10. Reconciliation of fiscal and monetary statistics remains a challenge. In the past, compilation of fiscal data solely on a cash basis and differences in the coverage of the central government explained most of the discrepancies between the datasets. In 2007, a TA mission found limited progress on previous recommendations on the fiscal reporting system for extra budgetary agencies and projects. The coverage of reporting agencies had improved, but there were many obvious errors and omissions in the data and in the BSL database. However, in 
June 2008 the authorities notified the IMF that the fiscal data have been reconciled with the expanded BSL monetary data.

\section{Balance of payments}

11. The BSL is responsible for compiling balance of payments (BOP) statistics. It obtains source data from the SSL, government ministries, the Customs and Excise Department (Customs), and the "Financial Survey of Major Limited Companies," for data on foreign direct investment. Imports and exports of goods estimates are based on data compiled by Customs and are adjusted for coverage, valuation, and timing to accord with BOP definitions. The BSL does not adjust BOP data using supplementary information to take account of unreported data.

12. External transactions are characterized by a large volume of activity in the informal sector, principally diamond smuggling. A considerable portion of imports is financed by these unrecorded exports. As a result, official BOP statistics tend to substantially understate transactions. Staff has been addressing this problem through the use of third-country (principally EU member) import data. STA has been providing technical assistance on BOP issues through the GDDS project to help the authorities implement the Balance of Payments Manual, Fifth Edition (BPM5).

13. Data problems also exist for trade in services, income statistics, current transfers, and the capital and financial accounts. Regarding the last, there are substantial difficulties in tracking financial transactions of the public and private sectors that are routed through commercial banks. While the authorities are producing data on the international investment position, improvements are required in coverage and in valuation adjustments. These difficulties are manifested in reconciling flow data in the BOP and stocks in the international investment position. Estimates of smuggled imports and exports, in particular diamonds, are also not available.

14. Information on official grant and loan receipts is relatively good and is prepared by AFR on the basis of contact with the authorities and donor agencies, but data on private capital flows are very poor. Some information on private banking flows can be derived from the monetary survey. Other private flows, especially those linked to the informal diamond trade, are implicitly included in "errors and omissions." 


\section{Sierra Leone: Table of Common Indicators Required for Surveillance} October 2009

\begin{tabular}{|c|c|c|c|c|c|}
\hline & $\begin{array}{l}\text { Date of latest } \\
\text { observation }\end{array}$ & Date received & $\begin{array}{l}\text { Frequency of } \\
\text { Data }^{4}\end{array}$ & $\begin{array}{l}\text { Frequency of } \\
\text { Reporting }^{4}\end{array}$ & $\begin{array}{l}\text { Frequency of } \\
\text { publication }^{4}\end{array}$ \\
\hline Exchange Rates & 10/08/09 & $10 / 15 / 09$ & $\mathrm{D}$ & W & W \\
\hline Reserve/Base Money & 09/09 & $10 / 15 / 09$ & M & M & M \\
\hline Consolidated Balance Sheet of the Banking System & $08 / 09$ & $10 / 05 / 09$ & M & M & M \\
\hline Interest Rates ${ }^{2}$ & 09/09 & $10 / 15 / 09$ & W & M & W \\
\hline Consumer Price Index & $08 / 09$ & 09/30/09 & M & M & M \\
\hline $\begin{array}{l}\text { Revenue, Expenditure, Balance and Composition of } \\
\text { Financing - Central Government }\end{array}$ & 06/09 & 08/31/09 & M & M & N/A \\
\hline $\begin{array}{l}\text { Stocks of Central Government and Central Government- } \\
\text { Guaranteed Debt }\end{array}$ & 06/09 & 08/31/09 & Q & Q & N/A \\
\hline External Current Account Balance & $06 / 09$ & 08/31/09 & A & A & A \\
\hline Exports and Imports of Goods and Services & 06/09 & $08 / 31 / 09$ & A & A & A \\
\hline GDP/GNP & $06 / 09$ & 08/31/09 & $A$ & A & A \\
\hline Gross External Debt & $06 / 09$ & 08/31/09 & A & A & A \\
\hline International Investment Position ${ }^{3}$ & 06/09 & 08/31/09 & $A$ & A & A \\
\hline
\end{tabular}

${ }^{1}$ Includes reserve assets pledged or otherwise encumbered as well as net derivative positions.

${ }^{2}$ Both market-based and officially-determined, including discount rates, money market rates, rates on treasury bills, notes and bonds.

${ }^{3}$ Includes external gross financial asset and liability positions vis-à-vis nonresidents.

${ }^{4}$ Daily (D), Weekly (W), Monthly (M), Quarterly (Q), Annually (A); Irregular (I); Not Available (NA). 
December 17, 2009

\section{IMF Executive Board Completes Fifth Review Under PRGF Arrangement with Sierra Leone, Approves US\$19.3 Million Disbursement, and Grants Waiver Related to Previous Noncomplying Disbursements}

The Executive Board of the International Monetary Fund (IMF) has completed the fifth review of Sierra Leone's economic performance under the Poverty Reduction and Growth Facility (PRGF) arrangement. Completion of the review, on December 16, 2009, will enable the immediate disbursement of SDR 12.185 million (US $\$ 19.3$ million), bringing total disbursements under the arrangement to SDR 44.88 million (about US\$71 million).

Performance under the authorities' program in the first half of 2009 was satisfactory. In completing its review, the Executive Board granted a waiver for nonobservance of the continuous performance criterion on the introduction or modification of multiple currency practices in relation to special windows - now eliminated - for rice and oil importers at its weekly foreign exchange auctions.

Falling global demand and declining foreign inflows negatively affected economic activity in the first half of 2009. However, diamond and agricultural production has rebounded lately. Although down from 5.5 percent in 2008, real gross domestic product (GDP) growth is expected to reach 4 percent in 2009. Lower fuel prices and higher domestic food production helped bring inflation down from double digits in 2008 to 8.3 percent in August 2009. However, there are upside risks to inflation after the recent depreciation of the leone against the US dollar.

The three-year PRGF arrangement was approved in May 2006 in the total amount of SDR 31.11 million (about US\$46 million, or 30 percent of quota-see Press Release 06/94). The arrangement was later extended by one year to May 9, 2010. Subsequently, two augmentations were approved for a total of SDR 20.77 million (about US\$33 million--see Press Releases $\underline{08 / 341}$ and $\underline{09 / 235}$ ). 
Following the discussion on Sierra Leone's economic performance, Murilo Portugal, Deputy Managing Director and Acting Chair, made the following statement:

“The Sierra Leonean authorities' commitment to sound macroeconomic policies has contributed to an improved economic outlook. Following a period of slowing global demand and declining inflows of remittances and foreign direct investment, indicators point to the strengthening economic activity in the second half of 2009, as the electricity supply rose, agricultural productivity improved, and spending on public infrastructure increased.

"Fiscal policy is targeted at improving revenue performance and containing spending pressures, while protecting social spending and expanding infrastructure outlays. The introduction of a goods and services tax in January 2010 will contribute to revenue enhancement, which will help create fiscal space for spending for poverty reduction and investment.

"The goal of monetary policy is to keep inflation in single digits and contain pressure on the exchange rate. To achieve this, it is important to explore options to expand short-term monetary instruments to increase flexibility in the conduct of monetary policy.

“The authorities' structural reform agenda appropriately focuses on deepening the financial sector, reforming the electricity sector, enhancing efficiency in the use of public resources, promoting good governance, and establishing a transparent and automatic pricing framework for petroleum products.

"A special window for oil importers in the foreign exchange auction in Sierra Leone resulted in a multiple currency practice, which was not reported to the Fund, thus giving rise to noncomplying disbursements under the PRGF arrangement. This special window has been eliminated and the Executive Board granted a waiver for the nonobservance of the associated performance criterion."

Sierra Leone joined the IMF in September 1962 and has a Fund quota of SDR 103.7 million. 


\section{Statement by Samuel Itam, Executive Director for Sierra Leone December 16, 2009}

\section{Introduction}

1. My Sierra Leonean authorities would like to thank staff for the fruitful policy dialogue in Freetown during the September 2009 mission in respect of the fifth review under the Poverty Reduction and Growth Facility (PRGF) arrangement. They are appreciative of the support of the Executive Board and Management in helping the country weather the adverse effects of the current global economic and financial meltdown. Notwithstanding the challenging external and domestic environments, Sierra Leone has remained steadfast to maintaining macroeconomic stability, sustaining economic growth, and reducing widespread poverty. My authorities broadly share the thrust of the staff report, which they consider to be a balanced account of recent economic developments and policy challenges going forward.

\section{Program performance}

2. Since steering the current PRGF-supported program back on track in early 2008, program implementation has improved significantly. Performance in 2009 has so far been impressive, as all the end-June 2009 quantitative performance criteria (PC) were met, including the target on domestic revenue which had in the past been very challenging. Substantial progress has also been made on the structural front, with reforms far advanced in the areas of public financial management, governance, financial sector reform, and the business climate. The structural benchmarks for end-June 2009 and endSeptember 2009 were also met, with the exception of one requiring revision of the underlying legislations for which my authorities are seeking technical assistance from the Fund.

3. However, as highlighted in the staff report and accompanying documentation, my authorities are requesting a waiver for the standard continuous performance criterion on the introduction or modification of multiple currency practices (MCPs). This emanated from the establishment of separate windows in the foreign exchange auction for two strategic commodity imports - oil and rice - in November 2008 and August 2009, respectively. Let me, on behalf of my authorities, reiterate that the breach of the said PC was inadvertent and that prompt corrective action was taken to eliminate the windows. As expressed in my authorities' response to the Managing Director's letter and the Letter of Intent, the segmentation of the market was part of a coordinated policy response to prevent scarcity of those essential commodities in a situation of acute shortage of foreign exchange spawned by the global financial crisis. This development did not alter the efficient conduct of the auction as it remained transparent, with continuous and full dissemination of information to market participants. My authorities have received technical assistance from the Fund since the establishment of the auction system and reaffirm their commitment to continue working with staff. In this regard, they are seeking further assistance to institute safeguards in the auction system to prevent any further inadvertent MCPs. 


\section{Recent economic developments}

4. My authorities' overall policy objectives of maintaining macroeconomic stability and promoting sustainable economic growth and development have been severely constrained by the adverse effects of the global crisis. With sluggish demand and falling export prices, coupled with declining inward remittances and FDI, real GDP growth is estimated at 4 percent in 2009 compared to 5.5 percent in 2008. The growth in output is supported by increased activities in agriculture, construction and services. However, there are signs of an early recovery with a pick up in diamond exports and an increase in agricultural productivity in the last quarter of 2009. Inflation was maintained at single digits during the first three quarters of 2009, largely on account of increasing domestic production and declining international prices for food and fuel. However, the passthrough from the depreciation of the leone has generated severe inflationary pressures.

5. Fiscal performance has gradually improved as a result of the efforts at broadening the tax base and strengthening tax administration. The authorities also exercised restraints in undertaking non-priority expenditures. Consequently, the fiscal deficit was contained within its end-June 2009 program target by about 0.5 percentage point of GDP.

6. The external current account widened in 2009 on account of the weak export performance, while the total value of imports has declined appreciably. The leone, which had been relatively stable over recent years, has depreciated markedly against major international currencies on account of declining foreign exchange inflows, even though demand has diminished. Gross foreign reserves at the end of October 2009, excluding the new SDR allocation, was equivalent to over 4 months of import cover.

\section{Challenges ahead and medium-term policies}

7. Following from their recent experience with the global food and fuel crisis and the dire effects of the current global crisis, my authorities are under no illusion regarding the magnitude of the challenges confronting the economy. The unrelenting fiscal pressures, depreciating exchange rate, heightening inflationary pressures, and weakening asset portfolio of the banking system call for measured, coordinated and synchronized policy responses over the medium term. My authorities' medium-term strategy is articulated in the President's Agenda for Change which forms the nucleus of the Second Generation Poverty Reduction Strategy Paper covering 2009 - 2012. It broadly aims at reducing the pervasive poverty by stimulating economic growth through increased investment in electricity, transportation, agriculture, and human development. At the same time, the authorities intend to continue with supporting measures to preserve macroeconomic stability. Renewed expectation of a rebound in domestic output and exports underpins a projected real GDP growth of 4.7 percent in 2010, increasing gradually to 6 percent in 2012. Inflation is envisaged to decline to 8 percent in 2010 and is projected to remain in single digits through 2012. As the pressure on the country's foreign reserves to stabilize the depreciating exchange rate wanes, a comfortable reserves position will be maintained over the medium term at above 5-6 months of import coverage. 


\section{Fiscal policy}

8. My authorities would like to reaffirm their commitment to prudent fiscal policy that enhances domestic revenue mobilization and rationalizes expenditures. Regarding the latter, emphasis will continue on productive capital and poverty related spending. On the revenue front, recent progress accomplished in modernizing the National Revenue Authority (NRA) will be consolidated. Substantial efficiency gains in tax collection are to be accrued from the recent installation of the ASYCUDA++ software at the Customs Department and the commencement of the goods and services tax (GST) on January 1, 2010. Further, the authorities will soon submit to Parliament amendments to the laws and regulations governing taxation of income and external trade that will reduce the opportunities for discretionary tax exemptions and provide for regular audit of exemptions. To underscore the determination to expeditiously pursue the fiscal agenda, the 2010 Finance Bill — which provides the legal basis for implementing new revenue measures - was, for the first time, submitted along side the 2010 budget to Parliament on December 4, 2009.

9. On the expenditure side, my authorities intend to restrain non-statutory recurrent expenditures while enhancing allocations to the priority sectors including roads, agriculture, energy, health and education. In furtherance of this goal, current expenditures relative to GDP will remain unchanged in 2010 , while domestically funded capital expenditures are to increase by over 1 percentage point of GDP. With support from the Fund and other development partners, notably the UK Department for International Development (DFID) and the World Bank, advances have been made in improving public financial management. The integrated financial management information system (IFMIS) has been rolled out to more government ministries, departments and agencies (MDAs). Also, implementation of an integrated public financial management reforms program is being fast-tracked in order to consolidate gains in improving the credibility, predictability, control and transparency of the budget.

\section{Monetary and Financial sector policies}

10. The current monetary targeting framework has served the country well in achieving relative price stability. Also, coordination between the monetary and fiscal authorities in policy formulation and implementation has improved. In June this year, the rediscount window at the Bank of Sierra Leone (BSL) was phased out in place of REPO/Reverse REPO operations that aim to deepen the inter-bank market. Going forward, my authorities will employ a more proactive monetary policy stance that preemptively prevents the build up of excess liquidity in the banking system. While experience with the central bank's liquidity forecasting and management framework has been favorable, efforts are being made to enhance the accuracy of its forecasts, including through closer cooperation with the Finance Ministry to ensure regular and timely provision of forward-looking expenditure profiles. 
11. The financial sector has generally demonstrated resilience in the face of the global financial meltdown. However, the authorities remain cognizant of the potential risks from the increasing accumulation of nonperforming loans by the commercial banks. In this regard, implementation of the recently adopted Financial Sector Development Plan (FSDP) aims at promoting financial intermediation, and strengthening the supervisory and legislative framework of the banking system expeditiously. Within this context, processes have been initiated to revise the Banking Act, the Financial Services Act, and the Anti-Money Laundering and Countering the Financing of Terrorism Act.

Furthermore, efforts are being made to operationalize a credit reference bureau that will seek to minimize the high rate of loan default. The recent commencement of trading at the Sierra Leone Stock Exchange, following its launch in July 2009, is expected to provide an avenue for long-term investment financing.

\section{Public debt management}

12. While the updated debt sustainability analysis jointly conducted by the Fund and World Bank reveals a moderate risk of debt distress, my authorities are mindful of the potential threats to sustainability, especially against the backdrop of a difficult external environment. They therefore commit to continue pursuing a prudent debt management policy to prevent relapse into debt overhang by inter alia concentrating on highly concessional loans. Plans are afoot to implement the second external commercial debtbuy-back operation to help address debt owed to commercial creditors. The World Bank has provided an initial funding of US\$950,000 under a Project Preparation Facility for the financing of advisory services to assist with the process. Furthermore, the authorities have secured financing from the African Development Bank to develop a National Debt Law that will provide a solid framework to ensure sound debt management in line with international best practices.

\section{Other structural reforms}

13. My authorities consider structural reforms an integral part of the Agenda for Change to promote delivery of social services and ensure efficiency, transparency and accountability in the use of public funds. Over and above the reforms already highlighted, the authorities will, seek to:

- $\quad$ strengthen the public procurement process by ensuring transparency in procurement activities and information dissemination, and improving public service records management;

- accelerate the formulation of a comprehensive electricity tariff policy and restructuring of the National Power Authority (NPA) to ensure financial viability and improved service delivery;

- consolidate progress accomplished in modernizing the business regulatory framework and eliminating administrative barriers to investment; and

- terminate the provision of subsidies to petroleum products. With effect from 2010, my authorities will re-institute a transparent automatic 
petroleum pricing mechanism anchored on a full pass-through of international oil prices and movements in the exchange rate.

14. My authorities want to acknowledge the support of our development partners which continues to be crucial. The World Bank is setting up a multi-donor trust fund in an effort to address the funding requirements for development of the country's infrastructure, as articulated in the Agenda for Change. The United Kingdom's Department for International Development (DFID) has already indicated its interest in participating in the Fund by contributing $£ 15$ million over a five year period for power sector investments. To better coordinate the external financial support, my authorities have formulated an aid policy that defines modalities for channeling aid to the country, with emphasis on better harmonization of donor support, and greater predictability of disbursements.

15. Finally, my authorities are delighted to inform the Executive Board of the resounding success of the Consultative Group meeting held in London on November 19, 2009 in support of the country's second generation poverty reduction strategy. It did not only serve as a forum to mobilize much-needed external financial resources in support of the medium-term development objectives, but was an opportunity to showcase the country's huge investment and tourist potentials. I wish to convey the gratitude of my authorities to multilateral and bilateral development partners for their continued financial support, and the government of the United Kingdom for hosting the conference.

\section{Conclusion}

16. My authorities recognize the enormous challenges of mobilizing domestic revenues, maintaining macroeconomic stability, and promoting pro-poor economic growth, particularly against the backdrop of the global crisis. They remain committed in their effort at creating an environment conducive to increasing the productive capacity of the economy for employment creation, poverty reduction, and the attainment of the MDGs. The authorities also consider IMF support as essential to achieving their development goals. As a result, program performance has steadily improved and all quantitative PCs in respect of the current review were met. Impressive progress has also been made with structural reforms - notably in public financial management, governance, financial sector development, and improving the business climate. The structural benchmarks for end-June 2009 and end-September 2009 were also met, with the exception of one, for which my authorities are seeking technical assistance from the Fund. The violation of the standard continuous PC on the introduction of multiple currency practices (MCPs) was unintentional. It is against this background that my authorities request a waiver for the nonobservance of the PC, completion of the fifth review under the PRGF arrangement, and modification of the quantitative PCs for endDecember 2009. 